\title{
Generalization of Geyer's commutation relations with respect to the orthogonal group in even dimensions
}

\author{
Yu. A. Markov ${ }^{\mathrm{a}}$, M. A. Markova ${ }^{\mathrm{b}}$ \\ Matrosov Institute for System Dynamics and Control Theory SB RAS, Irkutsk, Russia
}

Received: 5 August 2020 / Accepted: 23 October 2020 / Published online: 14 December 2020

(C) The Author(s) 2020

\begin{abstract}
A connection between the deformed DuffinKemmer-Petiau (DKP) algebra and an extended system of the parafermion trilinear commutation relations for the creation and annihilation operators $a_{k}^{ \pm}$and for an additional operator $a_{0}$ obeying para-Fermi statistics of order 2 based on the Lie algebra $\mathfrak{s o}(2 M+2)$ is established. An appropriate system of the parafermion coherent states as functions of paraGrassmann numbers is introduced. The representation for the operator $a_{0}$ in terms of generators of the orthogonal group $S O(2 M)$ correctly reproducing action of this operator on the state vectors of Fock space is obtained. A connection of the Geyer operator $a_{0}^{2}$ with the operator of so-called $G$-parity and with the $C P T$ - operator $\hat{\eta}_{5}$ of the DKP-theory is established. In a para-Grassmann algebra a noncommutative, associative star product $*$ (the Moyal product) as a direct generalization of the star product in the algebra of Grassmann numbers is introduced. Two independent approaches to the calculation of the Moyal product $*$ are considered. It is shown that in calculating the matrix elements in the basis of parafermion coherent states of various operator expressions it should be taken into account constantly that we work in the so-called Ohnuki and Kamefuchi's generalized state-vector space $\mathfrak{U}_{G}$, whose state vectors include para-Grassmann numbers $\xi_{k}$ in their definition, instead of the standard state-vector space $\mathfrak{U}$ (the Fock space).
\end{abstract}

\section{Introduction}

The principal purpose of the present paper is to study a connection between para-Fermi quantization based on the Lie algebra of the orthogonal group $S O(2 M+2)$, the DuffinKemmer-Petiau algebra with a deformation, and a paraGrassmann algebra. Note that an analysis of this connection is of particular mathematical interest without an application

\footnotetext{
a e-mail: markov@icc.ru (corresponding author)

be-mail: markova@icc.ru
}

to a specific physical problem, since the connection represents nontrivial synthesis of various subjects such as algebra, the theory of classical Lie groups and theoretical aspects of Green's parafields quantization.

There is a large number of papers devoted to various aspects of the DKP-formalism. Below we will mention just a few of them, which are concerned to the object of the given research.

The DKP-formalism describes spin-0 and spin-1 particles. The equation of motion represents the first order matrix-differential equation looking very similar to the Dirac equation. Analogue of Dirac's $\gamma$-matrices are so-called $\beta$ matrices obeying a more complicated trilinear relation [14]. Mathematical aspects of the DKP-algebra were studied in greater detail in the fundamental works by Kemmer [5], Harish-Chandra [6], Fujiwara [7], Tokuoka and Tanaka [8,9], Chernikov [10], Fischbach et al. [11,12], Filippov et al. [13], Isaev [14] etc. In particular, it was shown that the classification of representations of the DKP-algebra can be reduced to the classification of irreducible representations of the Lie algebra $\mathfrak{s o}(2 M+1)$ of the orthogonal group $S O(2 M+1)$. This DKP-algebra for physically more important case $M=2$ has 126 independent elements and admits the irreducible matrix representations of dimensions of 1 (trivial case), 5 and 10. Umezawa [15] has constructed the expressions for the projection operators on the sectors with spins 0 and 1 . Finally, it was shown that the DKP-algebra admits supersymmetric generalization [16].

Further, the Duffin-Kemmer-Petiau algebra is closely related to an entirely different branch of theoretical physics, namely, the theory of parastatistics, more exactly, to the paraFermi statistics of order $p=2$. This nontrivial fact was noted for the first time in the papers by Volkov [17], Chernikov [10] and independently by Ryan and Sudarshan [18]. This connection provided an opportunity to present the DKP-algebra within the framework of an operator formalism (see Sect. 4) in the form of parafermion algebra of order $p=2$ and to 
realize a spin space of vector particle as a Fock space for a system of para-Fermi operators [19].

However, a preliminary analysis [20] has shown that the use of parafermion algebra in the standard form is insufficient for solving some applied problems, for example in the construction of the path integral representation in parasuperspace for the Green's function of a spin-1 massive particle in external Maxwell's field, and here, a generalization of this algebra would be required. As is well known, trilinear commutation relations for the para-Fermi statistics generate algebra which is isomorphic to the Lie algebra $\mathfrak{s o}(2 M+1)$ [21]. Geyer in the paper [22] has suggested to extend this isomorphism to the Lie algebra $\mathfrak{s o}(2 M+2)$. The extension is of great value for us, since in the corresponding algebra of para-Fermi operators an additional operator $a_{0}$ arises. This operator in the case of parastatistics of order 2 can be related to within a sign to the Schrödinger "pseudomatrix" $\omega[23]$ playing a key role in constructing the divisor for the first order DKP-operator of a vector particle in an external gauge field [24]. This divisor allowed us in particular, to write an operator expression for the inverse propagator of a vector particle in the form of the Fock-Schwinger proper parasupertime representation. The Fock-Schwinger representation can be adopted as an initial expression for constructing the path-integral representation of the inverse propagator of the massive vector particle with the use of corresponding system of the para-Fermi coherent states.

In the construction of the necessary para-Fermi states the papers by Omote and Kamefuchi [25] and Ohnuki and Kamefuchi [26] are of particular interest for us. For a generalization of the notion of path integral to the case of parafermion variables in these papers the first step was to suggest an generalization of the well-known Grassmann algebra to the socalled para-Grassmann algebra [27]. This generalization is a direct analogue of generalization of the Fermi operators to the case of the para-Fermi operators in parastatistics. The authors have introduced the definition of the para-Grassmann algebra of arbitrary order $p$, the notions of integration and differentiation in this algebra, change of variables in integrals, Fourier transformation and so on. They also have defined the notions of coherent states for the para-Fermi operators and written out the formula of resolution of the identity (the completeness relation). Essentially all the mathematical apparatus constructed by these authors will be actively used in the suggested research.

In addition to the known results in the present work we would like to consider one more aspect of algebra of paraGrassmann numbers. In a para-Grassmann algebra for the first time we enter noncommutative and associative star product $*$ (the Moyal product), which in fact represents an integral convolution of a certain type of two para-Grassmann-valued functions. This is a straightforward generalization of a similar product in Grassmann algebra (see, for example, Bayen et al. [28], Tyutin [29], Smilga [30], Hirshfeld and Henselder [31], Daoud [32]). This product in our case arises naturally in calculating the matrix elements of a product of various operator expressions in the basis of parafermion coherent states. Moreover, the star product enables us to have a better understanding of a connection between the algebra of creation and annihilation operators $a_{k}^{ \pm}$of para-Fermi particles and the para-Grassmann algebra equipped with the product * making them in fact isomorphic as it takes place for the usual fermionic operators and Grassmann variables [32].

For calculating the star product operation we will use two different approaches. The first of them has somewhat heuristic character. It consists in separation by hands from various contributions to the integral only those which give nontrivial result after an integration. For separating these contributions we make use of a simple fact that the integral with respect to para-Grassmann variable $\mu$ of order $p$ is not vanishing only when the integrand contains this para-Grassmann variable exactly to the power of $p$ [26]. Another way was suggested in the paper by Omote and Kamefuchi [25]. The idea of this approach consists in reducing the integration over the paraGrassmann variables to calculating certain operator expressions averaged over the vacuum state. Here, ultimately, the calculation boils down to shift of the annihilation operators $a_{k}^{-}$to the right until the vacuum conditions can be employed. Here, the rearrangement rules are defined by the algebra of operators $a_{k}^{ \pm}$obeying the para-Fermi statistics of the special order $p$. Two independent approaches to calculating the product $*$ allows one to verify independently the results of calculations.

It is possible to make one more remark concerning algebra of the para-Grassmann variables. The para-Grassmann algebra of order $p=2$ in the spirit of the paper by Omote and Kamefuchi [25] is still quite visible at concrete calculations. However we can use another variant of the generalization of Grassmann variables also widely adopted (see, for example, Kwasniewski [33], Baulieu and Floratos [34], Fleury and Rausch de Traubenberg [35,36], Fillipov et al. [37-40]; Isaev [14]). These generalized Grassmann variables obey bilinear $q$-commutation relations, where $q$ is a primitive $n$th root of unity. The bilinear relations are simpler in comparison with the trilinear commutation relations for para-Grassmann variables and this significantly simplifies calculations with an increase of order $p$. In principle, the use of the generalized Grassmann algebra with the corresponding system of parafermion coherent states, the rules of integration and differentiation can be considered as an alternative to approach which is used in the present paper. It can be very important upon transition, for example, from the para-Grassmann algebra of order $p=2$ to significantly more difficult and more tangled algebra of order $p=3$. The latter is required for the description of particles with the spin $3 / 2$ as it was shown in one of our works [41]. 
One of the most interesting features of the approach developed in the present paper is the necessity of introduction instead of the standard Fock space $\mathfrak{U}$ an enlarged Fock space $\mathfrak{U}_{G}$, whose state vectors can also include the para-Grassmann numbers $\xi_{k}$. The need for such a generalization was first noted by Ohnuki and Kamefuchi [26] and it is connected with that the para-Fermi operators $a_{k}^{ \pm}$and the para-Grassmann numbers $\xi_{k}$ are not (anti)commutative for parastatistics of order $p \geq 2$. As a consequence, the parafermion coherent state (in the form which we use throughout this work) could not be represented in the form of an expansion in states with a certain number of para-Fermi particles. We discuss briefly this fact in Sect. 9. Careful consideration of this circumstance is required at all stages of calculations, or otherwise this leads to contradictions of various kinds. The necessity of considering Ohnuki and Kamefuchi's generalized state-vector space inevitably leads us to the need to introduce the quadratic Casimir operators $\hat{C}_{2}$ and $\hat{C}_{2}^{\prime}$ of the Lie algebras $\mathfrak{s o}(2 M)$ and $\mathfrak{s o}(2 M+1)$, correspondingly. In our specific case, when $p=2$ these operators are closely related to elements of the center of the Duffin-Kemmer-Petiau algebra as they were defined by Harish-Chandra [6]. In our derivation we use the representation of the quadratic Casimir operators in terms of the creation and annihilation operators $a_{k}^{ \pm}$of parafermions from the papers by Omote et al. [42] and by Bracken and Green [43] (see also Gould and Paldus [44]).

The paper is organized as follows. In Sect. 2, a brief review of Geyer's work [22] devoted to deriving the generalization of canonical commutation relations with respect to the orthogonal group $S O(n)$ in even dimensions, is presented. In Sect. 3, we give some formulas of the Duffin-Kemmer-Petiau theory: the trilinear relations for the $\beta_{\mu}$-matrices, the definition of Schrödinger's pseudomatrix $\omega$ and a cubic root of the unit matrix in term of the $\omega$. In Sect. 4, we provide all necessary formulas of operator formalism: the trilinear relations to which the second order parafermionic creation and annihilation operators obey, the basis of parafermion coherent states in the spin- 1 space $\mathcal{H}$, the normalization and completeness relations for the coherent states and so on. Section 5 is devoted to the calculation of the matrix element for the Geyer operator $a_{0}^{2}$, an analysis of its structure and derivation of its more compact and visual representation. In this section we have defined the resolvent operator $R$ of the $a_{0}^{2}$ on the basis of which an integral representation of the operator $a_{0}$ from the Lie algebra $\mathfrak{s o}(2 M+2)$ is written out. In Sect. 6 we have shown that this integral representation of the operator $a_{0}$ incorrectly reproduces action of this operator on the state vectors of the Fock space. In the same section another representation for the operator $a_{0}$ in terms of the generators of the group $S O(2 M)$ correctly reproducing action on the state vectors is suggested. A connection of this operator with the pseudoscalar DKP-operator $\hat{\omega}$ is obtained. Section 7 is concerned with the calculation of the matrix element for the operator $a_{0}$ in the basis of parafermion coherent states. At the end of this section a proof of the operator relation $a_{0}^{3}=a_{0}$ in terms of the matrix elements is given. In Sect. 8 a connection between the Harish-Chandra operator $\hat{\omega}^{2}$ and the Geyer operator $a_{0}^{2}$ is analyzed. As a secondary result the connection between the pseudoscalar DKP-operator $\hat{\omega}$ and the so-called $C P T$-operator $\hat{\eta}_{5}$ in the DKP theory is obtained.

Section 9 is devoted to establishing a connection between the Geyer operator $a_{0}^{2}$ and operator of so-called $G$-parity (the operator of parafermion parity $(-1)^{n}$, where $n$ is the parafermion number operator). In the same section, a brief analysis of a connection between two approaches in constructing the Lie algebra of the group $S O(2 M+2)$, namely, an approach of Geyer [22] and an approach of Fukutome [45], is performed. At the end of this section a feature of structure of Omote and Kamefuchi's definition for the para-Fermi coherent states is briefly discussed. Section 10 is devoted to the calculation of the matrix elements of the products $\hat{A} a_{n}^{ \pm}$ and of the commutators $\left[a_{0}, a_{n}^{ \pm}\right]$and $\left[a_{0}^{2}, a_{n}^{ \pm}\right]$, where $\hat{A} \equiv$ $\alpha \exp \left(-i \frac{2 \pi}{3} a_{0}\right)$. Two different forms of representation for the matrix elements of the commutators $\left[a_{0}^{2}, a_{n}^{ \pm}\right]$are considered. In Sect. 11 a similar calculation of the matrix elements of the product $\hat{A}\left[a_{0}, a_{n}^{ \pm}\right]$, is performed. The most compact representations for these matrix elements are defined. Sections 12 and 13 are devoted to the establishment of the relation between the functions $\Omega\left(\bar{\xi}^{\prime}, \xi\right)$ and $\widetilde{\Omega}\left(\bar{\xi}^{\prime}, \xi\right)$, which are the matrix elements of the operator $a_{0}$ and of the Geyer operator $a_{0}^{2}$ in the basis of parafermion coherent states. In Sect. 14 an important notion of the star product $*$ within the framework of the algebra of para-Grassmann numbers is introduced. In Sect. 15 the triple star product $\Omega * \Omega * \Omega$ of the function $\Omega=\Omega\left(\bar{\xi}^{\prime}, \xi\right)$ and the star exponential $\exp _{*}\left(-i \frac{2 \pi}{3} \Omega\right)$ are considered. In this section we have concluded ultimately that it is impossible to present the function $\widetilde{\Omega}\left(\bar{\xi}^{\prime}, \xi\right)$ as the star product of two functions $\Omega\left(\bar{\xi}^{\prime}, \xi\right)$, i.e. $\widetilde{\Omega} \neq \Omega * \Omega$.

In Sect. 16 to overcome a contradiction of the previous section, an analysis of the connection between the HarishChandra operator $\hat{\omega}^{2}$ and the Geyer operator $a_{0}^{2}$ is performed again and a more exact relation between these operators (in comparison with a similar relation obtained in Sect. 8) is derived. In Sect. 17 the quadratic Casimir operators $\hat{C}_{2}$ and $\hat{C}_{2}^{\prime}$ of the groups $S O(2 M)$ and $S O(2 M+1)$ are taken into consideration that enables one, in particular, to make a form of the connection between the operators $\hat{\omega}^{2}$ and $a_{0}^{2}$ more compact and explicit. The action rules of the Casimir operators on the state vectors, an explicit form of the matrix elements of these operators, and their representations through the operator $\hat{\Lambda} \equiv \sum_{k=1}^{2}\left\{a_{k}^{+}, a_{k}^{-}\right\}$are defined. Section 18 is devoted to discussion of the so-called generalized statevector space $\mathfrak{U}_{G}$ as it was defined by Ohnuki and Kamefuchi [26], whose state vectors include the para-Grassmann numbers $\xi_{k}$. Here we also briefly discuss a possibility of alternative definition of the parafermionic coherent states, 
which in principle allows to avoid introduction of the generalized state-vector space. In Sect. 19 the representation for the Harish-Chandra operator $\hat{\omega}^{2}$ in terms of the operator $\hat{\Lambda}$ is written out. In the same section it is proved that by using the refined connection between the operators $\hat{\omega}^{2}$ and $a_{0}^{2}$, we correctly reproduce the star product of three functions $\Omega$, namely $\Omega * \Omega * \Omega=\Omega$. In Sect. 20 the expressions for the commutators $\left[\hat{A}, a_{k}^{ \pm}\right]$and $\left[\hat{A},\left[a_{0}, a_{k}^{ \pm}\right]\right]$, are calculated. It is shown that the expressions obtained in calculating their matrix elements in the basis of the parafermion coherent states are contradictory with one another. It is pointed out that a reason of this contradiction is the use of trilinear commutation relation including two operators $a_{0}$ and one of the operators $a_{k}^{ \pm}$in the form as it was defined in the original paper by Geyer [22]. The most general form of this trilinear relation including the Casimir operator $\hat{C}_{2}^{\prime}$ is derived. The extended trilinear relation allows one to obtain finally the consistent expressions for the commutators $\left[\hat{A}, a_{k}^{ \pm}\right]$and $\left[\hat{A},\left[a_{0}, a_{k}^{ \pm}\right]\right]$. In the concluding Sect. 21 the key points of our work are specified.

In Appendix A all of the necessary formulas of algebra of the matrices $\omega$ and $\beta_{\mu}$ are listed. Appendix $\mathrm{B}$ is devoted to the formulation of the definition of a para-Grassmann algebra in a spirit of the paper by Omote and Kamefuchi [25]. The trilinear relations between the para-Grassmann numbers $\xi_{k}$ of order 2 and the creation and annihilation para-Fermi operators $a_{n}^{ \pm}$of parastatistics of order $p=2$ are also written out. In Appendix $C$ the formulas of differentiation with respect to the para-Grassmann variables used in the text of our paper are given. In Appendix D all of the necessary formulas of integration with respect to a para-Grassmann variable $\mu$ of order 2 are given. In Appendix $\mathrm{E}$ a list of the commutation relations between the generators $L_{k l}, M_{k l}$ and $N_{k l}$ of the group $S O(2 M)$ and between these generators and the operators $a_{n}^{ \pm}$ are written out.

In Appendix $\mathrm{F}$ we prove the validity of the operator relation $(-1)^{n} a_{0}=a_{0}$ based on an analysis of its matrix element or in other words we show that the matrix element of the operator $a_{0}$ in a basis of parafermion coherent states is even function with respect to change of the sign of paraGrassmann variables $\xi_{1}$ and $\xi_{2}$ (or $\bar{\xi}_{1}^{\prime}$ and $\bar{\xi}_{2}^{\prime}$ ) entering into the definition of the coherent states. In Appendix $\mathrm{G}$ we give a proof of turning into identity the commutation relations from Sect. 2 containing the operator $a_{0}$, when the latter is written in terms of the generators $L_{k l}, M_{k l}$ and $N_{k l}$. In Appendix $\mathrm{H}$ the derivation of the formula (68) given in the paper by HarishChandra [6] is considered again and its corrected expression is obtained. Finally, Appendix I is devoted to the proof of the relation $\left[a_{0}, \hat{\Lambda}\right]=0$. In particular, it is shown that in contrast to the relation $\left\{a_{0}, \hat{\Lambda}\right\}=8 a_{0}$, this operator relation does not fall into a sum of two independent relations $\left[a_{0}, \hat{\Lambda}_{i}\right]=0$, where $\hat{\Lambda}_{i} \equiv\left\{a_{i}^{+}, a_{i}^{-}\right\}, i=1,2$.

\section{Review of the Geyer work [22]}

A basis of an operator realization of the Lie algebra of the orthogonal group $S O(2 M+2)$ is given by the set of Hermitian operators

$I_{\mu \nu}=-I_{\nu \mu}$,

where the indices $\mu, v, \ldots$ run values $1,2, \ldots, 2 M+2$. These operators satisfy the commutation relations

$\left[I_{\mu \nu}, I_{\lambda \sigma}\right]=\delta_{\nu \lambda} I_{\mu \sigma}+\delta_{\mu \sigma} I_{\nu \lambda}-\delta_{\mu \lambda} I_{\nu \sigma}-\delta_{\nu \sigma} I_{\mu \lambda}$.

We introduce a new set of operators $\beta_{\mu}$ by setting ${ }^{1}$

$\beta_{\mu}=-i I_{\mu 2 M+2}$.

Here the index $\mu$ runs values $1,2, \ldots, 2 M+1$. The quantities $\beta_{\mu}$ are Hermitian

$\beta_{\mu}^{\dagger}=\beta_{\mu}$

and obey the commutation relations

$\left[\beta_{\mu}, \beta_{\nu}\right]=I_{\mu \nu}$,

$\left[\left[\beta_{\mu}, \beta_{\nu}\right], \beta_{\lambda}\right]=\beta_{\mu} \delta_{\nu \lambda}-\beta_{\nu} \delta_{\mu \lambda}$.

The property (2.1) enables us to introduce the Hermitian conjugate operators

$a_{k}^{+}=\beta_{2 k}-i \beta_{2 k-1}$,

$a_{k}^{-}=\beta_{2 k}+i \beta_{2 k-1}$,

where $k=1,2, \ldots, M$, and in addition to the $a_{k}^{ \pm}$, a further operator is defined as

$a_{0}=\beta_{2 M+1}\left(\equiv-i I_{2 M+1} 2 M+2\right)$.

The commutation relations between the operators $a_{k}^{ \pm}$are

$\left[a_{k}^{ \pm},\left[a_{m}^{\mp}, a_{n}^{ \pm}\right]\right]=2 \delta_{k m} a_{n}^{ \pm}$,

$\left[a_{k}^{ \pm},\left[a_{m}^{ \pm}, a_{n}^{ \pm}\right]\right]=0$,

$\left[a_{k}^{ \pm},\left[a_{m}^{\mp}, a_{n}^{\mp}\right]\right]=2 \delta_{k m} a_{n}^{\mp}-2 \delta_{k n} a_{m}^{\mp}$

and the commutation relations involving the operator $a_{0}$ are:

$\left[a_{k}^{ \pm},\left[a_{m}^{\mp}, a_{0}\right]\right]=2 \delta_{k m} a_{0}$,

$\left[a_{k}^{ \pm},\left[a_{m}^{ \pm}, a_{0}\right]\right]=0$,

$\left[a_{0},\left[a_{0}, a_{k}^{ \pm}\right]\right]=a_{k}^{ \pm}$,

$\left[a_{0},\left[a_{k}^{ \pm}, a_{m}^{\mp}\right]\right]=0$,

$\left[a_{0},\left[a_{k}^{ \pm}, a_{m}^{ \pm}\right]\right]=0$.

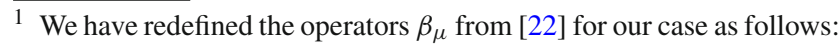
$\beta_{\mu} \rightarrow 2 \beta_{\mu}$ for $\mu=1,2, \ldots, 2 M+1$. 
Further, the uniqueness conditions of vacuum state $|0\rangle$ in the parastatistics of order $p$ are [46]:

$a_{k}^{-}|0\rangle=0$, for all $k$

and

$a_{k}^{-} a_{l}^{+}|0\rangle=p \delta_{k l}|0\rangle$, for all $k, l$.

The relation

$a_{0}|0\rangle= \pm \frac{1}{2} p|0\rangle$

is a consequence of requiring the uniqueness of the vacuum state. Note that the sign on the right-hand side of (2.14) may be chosen arbitrarily. Action of the operator $a_{0}$ on an arbitrary state vector

$$
|i j k \ldots r s\rangle=a_{i}^{+} a_{j}^{+} a_{k}^{+} \ldots a_{r}^{+} a_{s}^{+}|0\rangle
$$

is defined by the following formula:

$$
\begin{aligned}
& a_{0}|i j k \ldots r s\rangle= \pm \frac{1}{2} p|i j k \ldots r s\rangle \\
& \mp(|j k \ldots r s i\rangle+|i k \ldots r s j\rangle+\ldots \\
& +|i j k \ldots s r\rangle+|i j k \ldots r s\rangle) .
\end{aligned}
$$

In particular, this implies in addition to (2.14)

$$
\begin{aligned}
a_{0}|r\rangle & = \pm \frac{1}{2}(p-2)|r\rangle, \\
a_{0}|k r\rangle & = \pm \frac{1}{2}(p-2)|k r\rangle \mp|r k\rangle, \\
a_{0}|j k r\rangle & = \pm \frac{1}{2}(p-2)|j k r\rangle \mp(|j r k\rangle+|k r j\rangle), \\
a_{0}|i j k r\rangle & = \pm \frac{1}{2}(p-2)|i j k r\rangle \\
& \mp(|i j r k\rangle+|i k r j\rangle+|j k r i\rangle) .
\end{aligned}
$$

In the paper [22] a general relation for arbitrary values $p$ and $M$, which connects the operator $a_{0}$ with the operators $N_{1}, \ldots, N_{M}$ is also given (without a proof), where

$$
N_{k}=\frac{1}{2}\left[a_{k}^{+}, a_{k}^{-}\right]
$$

Let us write out the explicit form of the relations for the first three values $p$ in the case when $M=2$ :

$$
\begin{array}{cl}
p=1: & a_{0}=2 N_{1} N_{2}, \\
p=2: & a_{0}^{2}=\frac{1}{2}\left\{1+\left[2\left(N_{1}\right)^{2}-1\right]\left[2\left(N_{2}\right)^{2}-1\right]\right\}, \\
p=3: & a_{0}^{3}-\frac{7}{4} a_{0}=-\frac{1}{12} N_{1} N_{2}\left[4\left(N_{1}\right)^{2}-7\right] \\
& {\left[4\left(N_{2}\right)^{2}-7\right] .}
\end{array}
$$

\section{Duffin-Kemmer-Petiau formalism}

In the Duffin-Kemmer-Petiau theory the matrices $\beta_{\mu}$ obey the following trilinear relation:

$\beta_{\mu} \beta_{\nu} \beta_{\lambda}+\beta_{\lambda} \beta_{\nu} \beta_{\mu}=\delta_{\mu \nu} \beta_{\lambda}+\delta_{\lambda \nu} \beta_{\mu}$.

Let us now introduce the matrix $\omega$ setting by the definition in the even-dimension $D=2 M$ Euclidean space-time

$\omega=\frac{1}{(M !)^{2}} \epsilon_{\mu_{1} \mu_{2} \ldots \mu_{2 M}} \beta_{\mu_{1}} \beta_{\mu_{2}} \ldots \beta_{\mu_{2 M}}$.

This matrix plays an important part in further consideration. For the case when $M=2$ it was introduced into DKP theory for the first time by Schrödinger [23]. Here, we follow the notation used in the works by Harish-Chandra $[6,47,48]$, where the properties of the $\omega$ matrix were studied in detail. Let us note only that the matrix $\omega$ is identically zero for the spin 0, i.e. for the 5-dimensional irreducible representation of the DKP-algebra in the case of $M=2$. Therefore only the 10-row representation need be considered. In Appendix A all of the necessary formulas of algebra of the matrices $\omega$ and $\beta_{\mu}$ are listed.

In the paper [24] we have introduced a matrix $A$ in the form of the expansion in powers of $\omega$ :

$A=\alpha I+\beta \omega+\gamma \omega^{2}$

where the coefficients are

$\beta=\left(\frac{i \sqrt{3}}{2}\right) \alpha, \quad \gamma=\left(-\frac{3}{2}\right) \alpha, \quad \alpha^{3}=\frac{1}{m}$,

and $I$ is the unit matrix. The matrix $A$ meets the condition $A^{3}=\frac{1}{m} I$.

Thus the matrix $A / \alpha$ is a cubic root of the unit matrix. In the expansion (3.3) the property (A.1) was taken into account. Here, in addition we would like to give once more representation of the matrix (3.3), which sometimes is more convenient in concrete calculations. It is easy to show by using the property (A.1) that the following formula:

$\mathrm{e}^{i t \omega}=I+i \sin t \omega+(\cos t-1) \omega^{2}$,

where $t$ is an arbitrary real number, holds. In particular, for $t=2 \pi$ we have

$\mathrm{e}^{i 2 \pi \omega}=I$.

We are mainly interested in two important special cases:

1. in the case when $t=2 \pi / 3$, we have

$$
\alpha \mathrm{e}^{i \frac{2 \pi}{3} \omega}=\alpha\left(I+\frac{i \sqrt{3}}{2} \omega-\frac{3}{2} \omega^{2}\right) \equiv A,
$$


2 . in the case when $t=4 \pi / 3$, we have

$$
\alpha^{2} \mathrm{e}^{i \frac{4 \pi}{3} \omega}=\alpha^{2}\left(I-\frac{i \sqrt{3}}{2} \omega-\frac{3}{2} \omega^{2}\right) \equiv A^{2} .
$$

\section{The operator formalism}

Let us pass to an operator formulation of the DKP formalism. Instead of the $\beta_{\mu}$ matrices we enter operators $\hat{\beta}_{\mu}$. The operators $\hat{\beta}_{\mu}$ acts on the space $\mathcal{H}$ of the representation of the algebra

$\hat{\beta}_{\mu} \hat{\beta}_{\nu} \hat{\beta}_{\lambda}+\hat{\beta}_{\lambda} \hat{\beta}_{\nu} \hat{\beta}_{\mu}=\delta_{\mu \nu} \hat{\beta}_{\lambda}+\delta_{\lambda \nu} \hat{\beta}_{\mu}$.

The matrices $\beta_{\mu}$ are matrix elements of the operators $\hat{\beta}_{\mu}$ in the matrix basis $\{|\alpha\rangle ; \alpha=1,2, \ldots, 10\}$ in $\mathcal{H}$ :

$\left(\beta_{\mu}\right)_{\alpha \beta}=\left\langle\alpha\left|\hat{\beta}_{\mu}\right| \beta\right\rangle$.

For the case when $M=2$ within the framework of the operator formalism, instead of the general formula (3.2), we have

$\hat{\omega}=\frac{1}{4} \epsilon_{\mu \nu \lambda \sigma} \hat{\beta}_{\mu} \hat{\beta}_{\nu} \hat{\beta}_{\lambda} \hat{\beta}_{\sigma}$.

We will need a basis of coherent states in the spin- 1 space $\mathcal{H}$. In $\mathcal{H}$, the representation space of the Duffin-KemmerPetiau operator algebra (4.1), in accordance with (2.2) we introduce the parafermion creation and annihilation operators

$a_{1}^{ \pm}=\hat{\beta}_{2} \mp i \hat{\beta}_{1}, \quad a_{2}^{ \pm}=\hat{\beta}_{4} \mp i \hat{\beta}_{3}$.

These operators by virtue of (4.1) obey the following algebra:

$a_{k}^{ \pm} a_{l}^{\mp} a_{m}^{ \pm}+a_{m}^{ \pm} a_{l}^{\mp} a_{k}^{ \pm}=2 \delta_{k l} a_{m}^{ \pm}+2 \delta_{m l} a_{k}^{ \pm}$,

$a_{k}^{ \pm} a_{l}^{\mp} a_{m}^{\mp}+a_{m}^{\mp} a_{l}^{\mp} a_{k}^{ \pm}=2 \delta_{k l} a_{m}^{\mp}$,

$a_{k}^{ \pm} a_{l}^{ \pm} a_{m}^{ \pm}+a_{m}^{ \pm} a_{l}^{ \pm} a_{k}^{ \pm}=0, \quad k, l, m=1,2$

and the space $\mathcal{H}$ can be realized as a finite Fock space for the para-Fermi operators $\left(a_{1}^{ \pm}, a_{2}^{ \pm}\right)$.

As coherent states of the para-Fermi operators we take the coherent states as they were defined by Omote and Kamefuchi [25]. For parastatistics $p=2$ they have the form (in the case when $M=2$ ):

$$
\begin{aligned}
& \left|(\xi)_{2}\right\rangle=\exp \left(-\frac{1}{2} \sum_{l=1}^{2}\left[\xi_{l}, a_{l}^{+}\right]\right)|0\rangle, \\
& \left\langle\left(\bar{\xi}^{\prime}\right)_{2}\right|=\langle 0| \exp \left(\frac{1}{2} \sum_{l=1}^{2}\left[\bar{\xi}_{l}^{\prime}, a_{l}^{-}\right]\right),
\end{aligned}
$$

so that

$a_{k}^{-}\left|(\xi)_{2}\right\rangle=\xi_{k}\left|(\xi)_{2}\right\rangle, \quad\left\langle\left(\bar{\xi}^{\prime}\right)_{2}\right| a_{k}^{+}=\left\langle\left(\bar{\xi}^{\prime}\right)_{2}\right| \bar{\xi}_{k}^{\prime}$, where $\xi_{k}$ and $\bar{\xi}_{k}^{\prime}, k=1,2$, are para-Grassmann numbers obeying algebra (B.2). In Appendices B, C and D the formulation of the definition of a para-Grassmann algebra, the formulas for integration and differentiation with respect to a para-Grassmann variable of order 2 in a spirit of Omote and Kamefuchi [25] are given. For brevity sometimes we will write

$\sum_{l=1}^{2}\left[\xi_{l}, a_{l}^{+}\right] \equiv\left[\xi, a^{+}\right], \quad \sum_{l=1}^{2}\left[\bar{\xi}_{l}^{\prime}, \xi_{l}\right] \equiv\left[\bar{\xi}^{\prime}, \xi\right]$

and, moreover, since we are interested only in the case parastatistics of order 2, then we will omit the symbol 2 in the notation of the parafermion coherent states, i.e.

$\left|(\xi)_{2}\right\rangle \equiv|\xi\rangle, \quad\left\langle\left(\bar{\xi}^{\prime}\right)_{2}\right| \equiv\left\langle\bar{\xi}^{\prime}\right|$.

The overlap function and completeness relation for the coherent states (4.7) are given by

$$
\begin{aligned}
& \left\langle\bar{\xi}^{\prime} \mid \xi\right\rangle=\exp \left\{\frac{1}{2}\left[\bar{\xi}^{\prime}, \xi\right]\right\}, \\
& \iint|\xi\rangle\langle\bar{\xi}| \mathrm{e}^{-\frac{1}{2}[\bar{\xi}, \xi]}(d \xi)_{2}(d \bar{\xi})_{2}=\hat{1},
\end{aligned}
$$

where the measures of integration are defined by

$(d \xi)_{2} \equiv d^{2} \xi_{2} d^{2} \xi_{1}, \quad(d \bar{\xi})_{2} \equiv d^{2} \bar{\xi}_{1} d^{2} \bar{\xi}_{2}$.

The transition from the matrix elements in the coherent basis to the representation in which the DKP matrices $\beta_{\mu}$ have a specific form is realized as follows:

$$
\begin{aligned}
\langle\alpha|\ldots| \beta\rangle= & \iint \mathrm{e}^{-\frac{1}{2}\left[\bar{\xi}^{\prime}, \xi^{\prime}\right]}\left(d \xi^{\prime}\right)_{2}\left(d \bar{\xi}^{\prime}\right)_{2} \\
& \times \mathrm{e}^{-\frac{1}{2}[\bar{\xi}, \xi]}(d \xi)_{2}(d \bar{\xi})_{2}\left\langle\alpha \mid \xi^{\prime}\right\rangle \\
& \times\left\langle\bar{\xi}^{\prime}|\ldots| \xi\right\rangle\langle\bar{\xi} \mid \beta\rangle .
\end{aligned}
$$

The calculation of the explicit form of the transition functions $\langle\alpha \mid \xi\rangle$ and $\langle\bar{\xi} \mid \beta\rangle$ represents the nontrivial mathematical problem. This will be discussed elsewhere. Here we only point out that for this purpose the system of 20 algebraic equations should be written out and solved (for the case of spin $1 / 2$ the number of such equations equals 8 , see e.g. [49]). The coefficients of this system represent para-Grassmann numbers of order 2 .

\section{Matrix element of the operator $a_{0}^{2}$}

Our immediate task is the analysis of a connection between operators $\hat{\omega}$ and $a_{0}$, as they are defined by the equations (4.2) and (2.3), correspondingly. However as the first step we determine the matrix element for the operator $a_{0}^{2}$ in the basis of parafermion coherent states (4.7). The explicit form of this operator is given by Eq. (2.17). If one introduces the 
para-Fermi number operator of the $k$ state (for parastatistics $p=2$ )

$n_{k}=\frac{1}{2}\left[a_{k}^{+}, a_{k}^{-}\right]+1=N_{k}+1$,

then the expression (2.17) can be presented in the following equivalent form:

$$
\begin{aligned}
a_{0}^{2}= & 1-\left\{\left(n_{1}-1\right)^{2}+\left(n_{2}-1\right)^{2}\right\} \\
& +2\left(n_{1}-1\right)^{2}\left(n_{2}-1\right)^{2} .
\end{aligned}
$$

Let us determine action of the operator $a_{0}^{2}$ on the coherent state $|\xi\rangle$. For this purpose, we find a rule of action of the para-Fermi number operator $n_{k}$ on $|\xi\rangle$ :

$$
\begin{aligned}
n_{k}|\xi\rangle= & n_{k} \mathrm{e}^{-\frac{1}{2} \sum_{l}\left[\xi_{l}, a_{l}^{+}\right]}|0\rangle \\
= & {\left[n_{k}, \mathrm{e}^{-\frac{1}{2} \sum_{l}\left[\xi_{l}, a_{l}^{+}\right]}\right] } \\
& \times|0\rangle+\mathrm{e}^{-\frac{1}{2} \sum_{l}\left[\xi_{l}, a_{l}^{+}\right]} n_{k}|0\rangle .
\end{aligned}
$$

Here, the last term vanishes by virtue of the definition of vacuum state. The commutator in the first term is easily calculated by using the operator identity

$$
\begin{aligned}
\mathrm{e}^{X} Y \mathrm{e}^{-X}= & Y+[X, Y]+\frac{1}{2 !}[X,[X, Y]] \\
& +\frac{1}{3 !}[X,[X,[X, Y]]]+\cdots
\end{aligned}
$$

and commutation relations (B.3) and (B.4). This commutator equals $\frac{1}{2}\left[a_{k}^{+}, \xi_{k}\right]$ and thus we have

$n_{k}|\xi\rangle=\left(\frac{1}{2}\left[a_{k}^{+}, \xi_{k}\right]\right)|\xi\rangle$.

Recall that there is no summation over repeated Latin indices. Similar calculation for $n_{k}^{2}$ gives

$n_{k}^{2}|\xi\rangle=\left\{\frac{1}{2}\left[a_{k}^{+}, \xi_{k}\right]+\left(\frac{1}{2}\left[a_{k}^{+}, \xi_{k}\right]\right)^{2}\right\}|\xi\rangle$.

In view of the definition (5.2), it follows from (5.4) and (5.5) that

$$
\begin{aligned}
a_{0}^{2}|\xi\rangle= & \left\{1-\sum_{k=1}^{2}\left[\left(\frac{1}{2}\left[a_{k}^{+}, \xi_{k}\right]\right)^{2}-\frac{1}{2}\left[a_{k}^{+}, \xi_{k}\right]+1\right]\right. \\
& \left.+2 \prod_{k=1}^{2}\left[\left(\frac{1}{2}\left[a_{k}^{+}, \xi_{k}\right]\right)^{2}-\frac{1}{2}\left[a_{k}^{+}, \xi_{k}\right]+1\right]\right\}|\xi\rangle,
\end{aligned}
$$

and thus the required matrix element has the form

$$
\begin{aligned}
\left\langle\bar{\xi}^{\prime}\left|a_{0}^{2}\right| \xi\right\rangle= & \left\{1-\sum_{k=1}^{2}\left[\left(\frac{1}{2}\left[\bar{\xi}_{k}^{\prime}, \xi_{k}\right]\right)^{2}-\frac{1}{2}\left[\bar{\xi}_{k}^{\prime}, \xi_{k}\right]+1\right]\right. \\
& \left.+2 \prod_{k=1}^{2}\left[\left(\frac{1}{2}\left[\bar{\xi}_{k}^{\prime}, \xi_{k}\right]\right)^{2}-\frac{1}{2}\left[\bar{\xi}_{k}^{\prime}, \xi_{k}\right]+1\right]\right\} \\
& \times\left\langle\bar{\xi}^{\prime} \mid \xi\right\rangle .
\end{aligned}
$$

We analyze the structure of this expression in more detail. For the sake of convenience of further reasoning we introduce the notations:

$x \equiv \frac{1}{2}\left[\bar{\xi}_{1}^{\prime}, \xi_{1}\right], \quad y \equiv \frac{1}{2}\left[\bar{\xi}_{2}^{\prime}, \xi_{2}\right]$.

These variables by the algebra of para-Grassmann numbers (B.2) satisfy the following relations:

$x^{3}=0, \quad y^{3}=0, \quad x y=y x$.

In terms of $x$ and $y$, the matrix element (5.6) is written out in the form of a polynomial in $x$ and $y$ :

$$
\begin{aligned}
& \left\langle\bar{\xi}^{\prime}\left|a_{0}^{2}\right| \xi\right\rangle \\
& =\left[1-(x+y)+\left(x^{2}+y^{2}\right)+2 x y-2\left(x^{2} y+x y^{2}\right)\right. \\
& \left.\quad+2 x^{2} y^{2}\right]\left\langle\bar{\xi}^{\prime} \mid \xi\right\rangle .
\end{aligned}
$$

Here, we state the problem of representation of the expression in the square brackets in the form of the exponential of some function $\mathcal{U}$, which we present as

$$
\begin{aligned}
\mathcal{U}= & \mathcal{U}(x, y)=\alpha(x+y)+\beta\left(x^{2}+y^{2}\right)+\gamma x y \\
& +\delta\left(x^{2} y+y^{2} x\right)+\rho x^{2} y^{2},
\end{aligned}
$$

where $\alpha, \beta, \gamma \ldots$ are unknown coefficients. By virtue of algebra (5.8) we have

$\mathrm{e}^{\mathcal{U}}=1+\mathcal{U}+\frac{1}{2 !} \mathcal{U}^{2}+\frac{1}{3 !} \mathcal{U}^{3}+\frac{1}{4 !} \mathcal{U}^{4}$,

i.e. the power series exactly terminates with the fourthorder term. Let us substitute (5.10) into the right-hand side of (5.11), raise to the corresponding power with allowance for (5.8) and collect similar terms. Equating such obtained expression to the expression in the square brackets in (5.9), we get an algebraic system for the unknown coefficients:

$$
\begin{aligned}
& \alpha=-1, \quad \beta+\frac{1}{2} \alpha^{2}=1, \quad \gamma+\alpha^{2}=2, \\
& \delta+\alpha(\beta+\gamma)+\frac{1}{2} \alpha^{3}=-2, \\
& \rho+\frac{1}{2}\left(2 \beta^{2}+\gamma^{2}+4 \alpha \delta\right)+\alpha^{2}(\beta+\gamma)+\frac{1}{4} \alpha^{4}=2 .
\end{aligned}
$$

An unique solution of this system has the form

$\alpha=-1, \quad \beta=\frac{1}{2}, \quad \gamma=1, \quad \delta=0, \quad \rho=-\frac{1}{2}$

and thus

$$
\begin{aligned}
\mathcal{U} & =-(x+y)+\frac{1}{2}\left(x^{2}+y^{2}\right)+x y-\frac{1}{2} x^{2} y^{2} \\
& \equiv-(x+y)+\frac{1}{2}(x+y)^{2}-\frac{1}{12}(x+y)^{4} .
\end{aligned}
$$

If we remember the expression for the overlap function

$\left\langle\bar{\xi}^{\prime} \mid \xi\right\rangle=\mathrm{e}^{\frac{1}{2} \sum_{l}\left[\bar{\xi}_{l}^{\prime}, \xi_{l}\right]} \equiv \mathrm{e}^{x+y}$, 
then matrix element (5.9) goes into

$\left\langle\bar{\xi}^{\prime}\left|a_{0}^{2}\right| \xi\right\rangle=\mathrm{e}^{\mathcal{U}}\left\langle\bar{\xi}^{\prime} \mid \xi\right\rangle=\mathrm{e}^{\frac{1}{2}(x+y)^{2}-\frac{1}{12}(x+y)^{4}}$.

In spite of a more compact form in comparison with the initial expression (5.6), this formula is not convenient in concrete calculations by virtue of nonlinear character in $x$ and $y$ of the argument in the exponential. The form of the matrix element $\left\langle\bar{\xi}^{\prime}\left|a_{0}^{2}\right| \xi\right\rangle$ can be further simplified if we note that

$$
\begin{aligned}
& \mathrm{e}^{\frac{1}{2}(x+y)^{2}-\frac{1}{12}(x+y)^{4}} \\
& \quad=1+\frac{1}{2 !}(x+y)^{2}+\frac{1}{4 !}(x+y)^{4} \equiv \cosh (x+y),
\end{aligned}
$$

then finally we find

$\left\langle\bar{\xi}^{\prime}\left|a_{0}^{2}\right| \xi\right\rangle=\cosh \left(\frac{1}{2} \sum_{l}\left[\bar{\xi}_{l}^{\prime}, \xi_{l}\right]\right)$.

We return to the operator $a_{0}^{2}$ and analyze some of its properties. By analogy with (5.7) we introduce the notations

$\hat{x}=\frac{1}{2}\left[a_{1}^{+}, a_{1}^{-}\right], \quad \hat{y}=\frac{1}{2}\left[a_{2}^{+}, a_{2}^{-}\right]$.

Instead of algebra (5.8), now we have the operator algebra

$\hat{x}^{3}=\hat{x}, \quad \hat{y}^{3}=\hat{y}, \quad \hat{x} \hat{y}=\hat{y} \hat{x}$.

In terms of (5.14) the operator $a_{0}^{2}$ takes the form

$a_{0}^{2}=1-\left(\hat{x}^{2}+\hat{y}^{2}\right)+2 \hat{x}^{2} \hat{y}^{2}$.

Note that this operator is self-adjoint. Taking into account (5.15), it is not difficult to see that

$\left(a_{0}^{2}\right)^{2}=a_{0}^{2}\left(\equiv \mathcal{P}_{1}\right)$,

i.e. the operator has the property of a projector. Another projector orthogonal to $\mathcal{P}_{1}$ has the obvious form

$\mathcal{P}_{2} \equiv 1-a_{0}^{2}$.

It is worth pointing out that there exist one more structure orthogonal to $\mathcal{P}_{1}$, namely,

$\hat{x}+\hat{y}-\left(\hat{x}^{2} \hat{y}+\hat{x} \hat{y}^{2}\right)$,

which, however, doesn't possess the property of a projector.

Now we consider the problem of defining an explicit form of the resolvent of the operator $a_{0}^{2}$, i.e. of the operator $\left(a_{0}^{2}-\right.$ $\lambda)^{-1}$. For this purpose, we analyze the following equation:

$\left(a_{0}^{2}-\lambda\right)(\hat{\mathcal{U}}+\mu)=\hat{1}$,

where $\mu$ is unknown constant, and operator $\hat{\mathcal{U}}$ is defined by expression (5.10) with the replacements $x \rightarrow \hat{x}, y \rightarrow \hat{y}$. Equation (5.17) with algebra (5.15) results in a simple system of algebraic equations for the unknown coefficients in (5.10)

$\mu(1-\lambda)=1, \quad \alpha=0, \quad \mu+\lambda \beta=0, \quad \gamma(1-\lambda)=0$,

$\alpha+\delta(1-\lambda)=0, \quad \rho+2 \beta+2 \mu-\lambda \rho=0$, whose solution is

$\mu=\frac{1}{1-\lambda}, \quad \beta=-\frac{1}{\lambda(1-\lambda)}, \quad \rho=\frac{2}{\lambda(1-\lambda)}$,

$\alpha=\delta=\gamma=0$.

Hence, the resolvent of the operator $a_{0}^{2}$ has the form

$R_{\lambda}=\frac{1}{1-\lambda}\left\{\hat{1}-\frac{1}{\lambda}\left(1-a_{0}^{2}\right)\right\}$.

The resolvent is defined for all values of the parameter $\lambda$ with the exception of two points: 0 and 1 , i.e. the spectrum is $\sigma\left(a_{0}^{2}\right)=\{0,1\}$. In particular, it immediately follows that the operator $a_{0}^{2}$ is irreversible. Further, we can define an arbitrary analytic function of $a_{0}^{2}$ using for this purpose the representation [50]

$\varphi\left(a_{0}^{2}\right)=-\frac{1}{2 \pi i} \oint_{\Gamma_{a_{0}^{2}}} \varphi(\lambda) R_{\lambda}\left(a_{0}^{2}\right) d \lambda$,

where the contour $\Gamma_{a_{0}^{2}}$ surrounds the spectrum $\sigma\left(a_{0}^{2}\right)$. We are interested in the special case of choosing the function $\varphi$

$\varphi(\lambda)=\sqrt{\lambda}$

then

$a_{0}=-\frac{1}{2 \pi i} \oint_{\Gamma_{a_{0}^{2}}} \frac{\sqrt{\lambda}}{1-\lambda}\left\{\hat{1}-\frac{1}{\lambda}\left(1-a_{0}^{2}\right)\right\} d \lambda$,

i.e. formally we have the expression for the operator $a_{0}$, which enters into the commutation relations (2.7)-(2.11) and in the condition (2.14) on the vacuum state vector $|0\rangle$. By the spectral mapping theorem [50] the spectrum of this operator is $\sigma\left(a_{0}\right)=\left[\sigma\left(a_{0}^{2}\right)\right]^{1 / 2}=\{0, \pm 1\}$.

Let us rewrite expression (5.18) in a somewhat different form

$$
\begin{aligned}
a_{0}= & \frac{1}{2 \pi i} \oint_{\Gamma_{a_{0}^{2}}} \frac{d \lambda}{\sqrt{\lambda}} \hat{1} \\
& +\left\{-\frac{1}{2 \pi i} \oint_{\Gamma_{a_{0}^{2}}} \frac{d \lambda}{\sqrt{\lambda}}+\frac{1}{2 \pi i} \oint_{\Gamma_{a_{0}^{2}}} \frac{\sqrt{\lambda} d \lambda}{\lambda-1}\right\} a_{0}^{2} .
\end{aligned}
$$

In the next section we examine the question of action of the operator $a_{0}$ on state vectors of the system under consideration. Here, we consider only action on the ground state. It follows from the expression (5.2) that

$a_{0}^{2}|0\rangle=|0\rangle$,

and the condition (2.14) for $p=2$ yields

$a_{0}|0\rangle= \pm|0\rangle$. 
On the other hand from the representation (5.19) by virtue of (5.20) it follows that

$a_{0}|0\rangle=\left(\frac{1}{2 \pi i} \oint_{\Gamma_{a_{0}^{2}}} \frac{\sqrt{\lambda} d \lambda}{\lambda-1}\right)|0\rangle$.

Hence, uncertainty in the sign in the condition (5.21) is connected with twovaluedness of the function $\lambda^{1 / 2}$ in the domain of $0<|\lambda|<\infty$. Indeed, let us consider the contour $\Gamma_{a_{0}^{2}}$ consisting of the circle $|\lambda|=2$ and the segments $[-2,0]$ and $[0,-2]$, which lie on the upper and lower banks, respectively. The function $\sqrt{\lambda}$ splits in the domain into two regular branches, $g_{1}(\lambda)$ and $g_{2}(\lambda)$. This means that the integrand splits into two regular branches, $f_{1}(\lambda)=g_{1}(\lambda) /(\lambda-1)$ and $f_{2}(\lambda)=g_{2}(\lambda) /(\lambda-1)$. Let $g_{1}(\lambda)$ be the branch of the root on which $g_{1}(1)=1$, then $g_{2}(1)=-1$. Each function $f_{1,2}(\lambda)$ is regular in the domain being considered except at point $\lambda=1$, which is a simple pole. By the residue theorem we have

$\frac{1}{2 \pi i} \oint_{\Gamma_{a_{0}^{2}}} \frac{\sqrt{\lambda} d \lambda}{\lambda-1}= \pm 1$

\section{Operator $a_{0}$}

We turn now to the to construction of an explicit form of the operator $a_{0}$ in terms of the parafermion creation and annihilation operators $a_{k}^{ \pm}$. For convenience of further reference we write out all independent state vectors spanned by the operators $a_{k}^{ \pm}$. In our case, when $M=2$ according to Chernikov [10] the number of these state vectors equals $C_{5}^{2}=10$, and the number of para-Fermi particles in each state is

$n_{0}=C_{2}^{0} C_{2}^{0}=1, \quad n_{1}=C_{2}^{1} C_{2}^{0}=2, \quad n_{2}=C_{2}^{1} C_{2}^{1}=4$,

$n_{3}=C_{2}^{2} C_{2}^{1}=2, \quad n_{4}=C_{2}^{2} C_{2}^{2}=1$.

These states are

$$
\begin{array}{ll}
|0\rangle, & \\
|1\rangle \equiv a_{1}^{+}|0\rangle, & |2\rangle \equiv a_{2}^{+}|0\rangle, \\
|11\rangle \equiv\left(a_{1}^{+}\right)^{2}|0\rangle, & |22\rangle \equiv\left(a_{2}^{+}\right)^{2}|0\rangle, \\
|12\rangle \equiv a_{1}^{+} a_{2}^{+}|0\rangle, & |21\rangle \equiv a_{2}^{+} a_{1}^{+}|0\rangle, \\
|112\rangle \equiv\left(a_{1}^{+}\right)^{2} a_{2}^{+}|0\rangle, & |221\rangle \equiv\left(a_{2}^{+}\right)^{2} a_{1}^{+}|0\rangle, \\
|1122\rangle \equiv\left(a_{1}^{+}\right)^{2}\left(a_{2}^{+}\right)^{2}|0\rangle . &
\end{array}
$$

All the remaining states are a consequence of (6.1) by virtue of algebra ${ }^{2}(4.4)-(4.6)$. The states (6.1) can be written in socalled standard form [51-54] (see also [55]), however, we will not do so. We restrict oneself to consideration of the

\footnotetext{
2 In particular, one has

$|121\rangle=|212\rangle=0, \quad|211\rangle=-|112\rangle, \quad|221\rangle=-|122\rangle$,

$|2211\rangle=|1122\rangle=-|2112\rangle=-|1221\rangle$.
}

simple representation (6.1) of the parastatistical Fock space. In addition, we write out the norms of the state vectors

$$
\begin{aligned}
& \langle 0 \mid 0\rangle=1, \quad\langle l \mid k\rangle=2 \delta_{k l}, \quad\langle l k \mid m n\rangle=2^{2} \delta_{k m} \delta_{l n}, \\
& \langle l k k \mid k k l\rangle=2^{3}\left(1-\delta_{k l}\right), \quad\langle l l k k \mid k k l l\rangle=2^{4}\left(1-\delta_{k l}\right) .
\end{aligned}
$$

By virtue of the definition (5.2) we immediately obtain

$$
\begin{aligned}
& a_{0}^{2}|0\rangle=|0\rangle, \quad a_{0}^{2}|k\rangle=0, \\
& a_{0}^{2}|k k\rangle=|k k\rangle, \quad a_{0}^{2}|k k l\rangle=0, \\
& a_{0}^{2}|k l\rangle=|k l\rangle, \\
& a_{0}^{2}|k k l l\rangle=|k k l l\rangle, k \neq l, \quad k, l=1,2,
\end{aligned}
$$

i.e. the operator $a_{0}^{2}$ turns into zero the states with an odd number of parafermions.

Further we define the rules of an action of the operator $a_{0}$ on the state vectors (6.1). For definiteness we fix the positive sign in formula (2.14), i.e. we set

$a_{0}|0\rangle=|0\rangle$.

Then from general relations (2.15) with allowance for the algebra (4.4)-(4.6) it follows that

$a_{0}|1\rangle=a_{0}|2\rangle=0$,

$a_{0}|11\rangle=-|11\rangle, \quad a_{0}|22\rangle=-|22\rangle$,

$a_{0}|12\rangle=-|21\rangle, \quad a_{0}|21\rangle=-|12\rangle$,

$a_{0}|112\rangle=a_{0}|221\rangle=0$,

$a_{0}|1122\rangle=|1122\rangle$.

The operator $a_{0}$ similar to the operator $a_{0}^{2}$ turns into zero states with an odd number of parafermions. The signs on the right-hand side (6.5), (6.6) and (6.8) are connected with a choice of the sign in (2.14). The relations (6.6) are of special interest. Two different states $|12\rangle$ and $|21\rangle$ are orthogonal to each other and contain the same number of parafermions of sorts 1 and 2, i.e. the two-particle system has a two-fold degeneracy. The operator $a_{0}$ correct to a sign changes one state to another.

From the other hand, if we act by the operator $a_{0}$ in the representation (5.19) on the state vectors (6.1), then in view of (6.2) for the states with an odd number of paraparticles we have

$$
\begin{aligned}
a_{0}|k\rangle & =\left(\frac{1}{2 \pi i} \oint_{\Gamma_{a_{0}^{2}}} \frac{d \lambda}{\lambda^{1 / 2}}\right)|k\rangle, \\
a_{0}|k k l\rangle & =\left(\frac{1}{2 \pi i} \oint_{\Gamma_{a_{0}^{2}}} \frac{d \lambda}{\lambda^{1 / 2}}\right)|k k l\rangle, k \neq l
\end{aligned}
$$

and for states with an even number of paraparticles we get

$$
a_{0}|k k\rangle=\left(\frac{1}{2 \pi i} \oint_{\Gamma_{a_{0}^{2}}} \frac{\lambda^{1 / 2} d \lambda}{\lambda-1}\right)|k k\rangle,
$$




$$
\begin{aligned}
a_{0}|k l\rangle & =\left(\frac{1}{2 \pi i} \oint_{\Gamma_{a_{0}^{2}}} \frac{\lambda^{1 / 2} d \lambda}{\lambda-1}\right)|k l\rangle, \\
a_{0}|k k l l\rangle & =\left(\frac{1}{2 \pi i} \oint_{\Gamma_{a_{0}^{2}}} \frac{\lambda^{1 / 2} d \lambda}{\lambda-1}\right)|k k l l\rangle .
\end{aligned}
$$

If we fix the positive branch in the integral (5.22)

$$
\frac{1}{2 \pi i} \oint_{\Gamma_{a_{0}^{2}}} \frac{\lambda^{1 / 2} d \lambda}{\lambda-1}=+1
$$

for consistency with our choice of the sign in (6.3), and besides simply set for the second ${ }^{3}$ integral

$$
\frac{1}{2 \pi i} \oint_{\Gamma_{a_{0}^{2}}} \frac{d \lambda}{\lambda^{1 / 2}}=0
$$

then we reproduce relations (6.3), (6.4), (6.7) and (6.8). However, differences in the signs for (6.5), and most importantly, complete disagreement with (6.6), take place. The possible reason for this lies in the fact that the expression for the operator $a_{0}^{2}$, Eq. (5.2), suggested by Geyer [22] is not likely to be the square of the operator $a_{0}$, i.e. in other words,

$\left(a_{0}^{2}\right)^{1 / 2} \neq a_{0}$,

and thus the representation (5.18) is not correct. This delicate matter will be discussed in detail in the subsequent sections.

In the remainder of this section we consider approach to defining an explicit form of the operator $a_{0}$ based on making use of the generators $L_{k l}, M_{k l}$ and $N_{k l}$ of the group $S O(2 M)$ as they are defined in Appendix E, Eq.(E.1). In the special case $M=2$ we have the following components of these generators different from zero:

$$
\begin{array}{ll}
L_{12}=\frac{1}{2}\left[a_{1}^{+}, a_{2}^{+}\right], & M_{12}=\frac{1}{2}\left[a_{1}^{-}, a_{2}^{-}\right], \\
N_{12}=\frac{1}{2}\left[a_{1}^{+}, a_{2}^{-}\right], & N_{21}=\frac{1}{2}\left[a_{2}^{+}, a_{1}^{-}\right], \\
N_{1}=\frac{1}{2}\left[a_{1}^{+}, a_{1}^{-}\right], & N_{2}=\frac{1}{2}\left[a_{2}^{+}, a_{2}^{-}\right]
\end{array}
$$

and the general commutation relations (E.2) take a simple form

\footnotetext{
3 This integral is badly defined since one of points of the spectrum $\sigma\left(a_{0}^{2}\right)$ is the branch point for the function $\lambda^{1 / 2}$. It can be trivially estimated as follows. As the contour $\Gamma_{a_{0}^{2}}$ we take the circle $C_{R}:|\lambda|=R$. Further, we set $\lambda=R e^{i \varphi}$ and therefore $d \lambda=i R e^{i \varphi} d \varphi, \lambda^{1 / 2}=$ $R^{1 / 2} e^{i \varphi / 2+i \pi n}, n=0,1$. Purely formal calculation results in the expression
}

$\oint_{|\lambda|=R} \frac{d \lambda}{\lambda^{1 / 2}}=i \sqrt{R} \int_{0}^{2 \pi} \mathrm{e}^{i \varphi / 2-i \pi n} d \varphi=-4 \sqrt{R} \mathrm{e}^{-i \pi n}$,

which vanishes only in the limit $R \rightarrow 0$.

$$
\begin{aligned}
& {\left[N_{12}, N_{21}\right]=N_{1}-N_{2},} \\
& {\left[L_{12}, M_{12}\right]=-\left(N_{1}+N_{2}\right),} \\
& {\left[L_{12}, N_{12}\right]=\left[L_{12}, M_{21}\right]=0,} \\
& {\left[M_{12}, N_{12}\right]=\left[N_{12}, M_{21}\right]=0,} \\
& {\left[L_{12}, N_{1}\right]=-L_{12}, \quad\left[L_{12}, N_{2}\right]=-L_{12},} \\
& {\left[M_{12}, N_{1}\right]=M_{12}, \quad\left[M_{12}, N_{2}\right]=M_{12},} \\
& {\left[N_{12}, N_{1}\right]=-N_{12}, \quad\left[N_{12}, N_{2}\right]=N_{12},} \\
& {\left[N_{21}, N_{1}\right]=-N_{21}, \quad\left[N_{21}, N_{2}\right]=N_{21} .}
\end{aligned}
$$

By using the definition (6.9) and the algebra (4.4)-(4.6) it is easy to check the validity of the following relations:

$$
\begin{aligned}
& L_{12} M_{12}|12\rangle=M_{12} L_{12}|12\rangle=|21\rangle-|12\rangle, \\
& L_{12} M_{12}|21\rangle=M_{12} L_{12}|21\rangle=|12\rangle-|21\rangle
\end{aligned}
$$

and, correspondingly,

$$
\begin{aligned}
& N_{12} N_{21}|12\rangle=N_{21} N_{12}|12\rangle=|12\rangle+|21\rangle, \\
& N_{21} N_{12}|21\rangle=N_{12} N_{21}|21\rangle=|12\rangle+|21\rangle .
\end{aligned}
$$

If one accepts that the operator $a_{0}$ has the following structure:

$a_{0} \sim-\frac{1}{4}\left(\left\{L_{12}, M_{12}\right\}+\left\{N_{12}, N_{21}\right\}\right)$,

then we obtain

$a_{0}|12\rangle=-|21\rangle, \quad a_{0}|21\rangle=-|12\rangle$.

By doing so, we reproduce equality (6.6). However, the action of the operator (6.12) on vacuum state vector gives us

$a_{0}|0\rangle=\frac{1}{2}|0\rangle$

that is in contradiction with (6.3). To determine the action of the operator (6.12) on the other state vectors, we need the rules of commutation of the group generators (6.9) with the operators $a_{k}^{ \pm}$. These rules follow from general relations (E.3) for $M=2$ :

$$
\begin{array}{ll}
{\left[a_{k}^{-}, L_{12}\right]=\delta_{k 1} a_{2}^{+}-\delta_{k 2} a_{1}^{+},} & {\left[a_{k}^{-}, M_{12}\right]=0,} \\
{\left[a_{k}^{+}, M_{12}\right]=\delta_{k 1} a_{2}^{-}-\delta_{k 2} a_{1}^{-},} & {\left[a_{k}^{+}, L_{12}\right]=0,} \\
{\left[a_{k}^{-}, N_{12}\right]=\delta_{k 1} a_{2}^{-},} & {\left[a_{k}^{+}, N_{12}\right]=-\delta_{k 2} a_{1}^{+},} \\
{\left[a_{k}^{-}, N_{21}\right]=\delta_{k 2} a_{1}^{-},} & {\left[a_{k}^{+}, N_{21}\right]=-\delta_{k 1} a_{2}^{+},} \\
{\left[a_{l}^{-}, N_{k}\right]=\delta_{k l} a_{l}^{-},} & {\left[a_{l}^{+}, N_{k}\right]=-\delta_{k l} a_{l}^{+} .}
\end{array}
$$

Based on these relations and the rules of action on the vacuum state (6.14), it is not difficult to obtain for (6.12)

$$
\begin{array}{ll}
a_{0}|1\rangle=a_{0}|2\rangle=0, & a_{0}|112\rangle=0, \\
a_{0}|11\rangle=-\frac{1}{2}|11\rangle, & a_{0}|221\rangle=0, \\
a_{0}|22\rangle=-\frac{1}{2}|22\rangle, & a_{0}|1122\rangle=\frac{1}{2}|1122\rangle .
\end{array}
$$

Here, we also observe appearance of undesirable factor $\frac{1}{2}$ on the right-hand side as it takes place in (6.14). 
Let us take, instead of (6.12), the operator

$a_{0}=-\frac{1}{4}\left(\left\{L_{12}, M_{12}\right\}+\left\{N_{12}, N_{21}\right\}\right)+U\left(N_{1}, N_{2}\right)$.

We choose an operator function $U\left(N_{1}, N_{2}\right) \equiv U\left(n_{1}-1, n_{2}-\right.$ 1) so that the operator $a_{0}$ would reproduce correctly relations (6.14) and (6.16), while retaining (6.13). As a general expression for $U$ one takes (5.10) with the replacements $x \rightarrow n_{1}-1$ and $y \rightarrow n_{2}-1$. We obtain the following system of linear algebraic equations for the unknown coefficients in (5.10):

$$
\begin{array}{ll}
|0\rangle: & U(-1,-1)=-2 \alpha+2 \beta+\gamma-2 \delta+\rho=\frac{1}{2}, \\
|1\rangle,|2\rangle: & U(0,-1)=U(-1,0)=-\alpha+\beta=0, \\
|11\rangle,|22\rangle: & U(1,-1)=U(-1,1)=2 \beta-\gamma+\rho=-\frac{1}{2}, \\
|12\rangle,|21\rangle: & U(0,0) \equiv 0, \\
|112\rangle,|221\rangle: & U(1,0)=U(0,1)=\alpha+\beta=0, \\
|1122\rangle: & U(1,1)=2 \alpha+2 \beta+\gamma+2 \delta+\rho=\frac{1}{2} .
\end{array}
$$

The solution of this system is

$\alpha=\beta=\delta=\rho=0, \quad \gamma=\frac{1}{2}$,

and therefore, the desired operator function $U$ has the form

$U\left(N_{1}, N_{2}\right)=\frac{1}{2} N_{1} N_{2} \equiv \frac{1}{4}\left\{N_{1}, N_{2}\right\}$.

Thus, the operator $a_{0}$ as a function of the generators (6.9), correctly reproducing the relations (6.3)-(6.8), is of the following final structure:

$a_{0}=-\frac{1}{4}\left(\left\{L_{12}, M_{12}\right\}+\left\{N_{12}, N_{21}\right\}-\left\{N_{1}, N_{2}\right\}\right)$.

In closing this section let us define a connection between the operators $a_{0}$ and $\hat{\omega}$. We rewrite the expression for $\hat{\omega}$, Eq. (4.2), in an equivalent form:

$$
\begin{aligned}
\hat{\omega}= & \left(\frac{1}{4}\right)^{2} \epsilon_{\mu \nu \lambda \sigma}\left[\hat{\beta}_{\mu}, \hat{\beta}_{\nu}\right]\left[\hat{\beta}_{\lambda}, \hat{\beta}_{\sigma}\right] \\
= & \frac{1}{4}\left(\left\{\left[\hat{\beta}_{1}, \hat{\beta}_{2}\right],\left[\hat{\beta}_{3}, \hat{\beta}_{4}\right]\right\}+\left[\hat{\beta}_{1}, \hat{\beta}_{4}\right],\left[\hat{\beta}_{2}, \hat{\beta}_{3}\right]\right\} \\
& \left.\left.-\left[\hat{\beta}_{1}, \hat{\beta}_{3}\right],\left[\hat{\beta}_{2}, \hat{\beta}_{4}\right]\right\}\right) .
\end{aligned}
$$

Further, we write down the expression in the last two lines in terms of the creation and annihilation operators by using the connection (4.3) and the definitions (6.9). It is easy to show that the following relations hold:

$$
\begin{aligned}
& {\left[\hat{\beta}_{1}, \hat{\beta}_{2}\right]=i N_{1}, \quad\left[\hat{\beta}_{3}, \hat{\beta}_{4}\right]=i N_{2},} \\
& {\left[\hat{\beta}_{1}, \hat{\beta}_{4}\right]=\frac{1}{2 i}\left[\left(L_{12}-M_{12}\right)-\left(N_{12}+N_{21}\right)\right],} \\
& {\left[\hat{\beta}_{2}, \hat{\beta}_{3}\right]=\frac{1}{2 i}\left[\left(L_{12}-M_{12}\right)+\left(N_{12}+N_{21}\right)\right],} \\
& {\left[\hat{\beta}_{1}, \hat{\beta}_{3}\right]=\frac{1}{2}\left[\left(L_{12}+M_{12}\right)+\left(N_{12}-N_{21}\right)\right],} \\
& {\left[\hat{\beta}_{2}, \hat{\beta}_{4}\right]=-\frac{1}{2}\left[\left(L_{12}+M_{12}\right)-\left(N_{12}-N_{21}\right)\right] .}
\end{aligned}
$$

Substituting these expressions into (6.18), we obtain an explicit form of the operator $\hat{\omega}$ in terms of generators of the orthogonal group $S O(4)$

$\hat{\omega}=\frac{1}{4}\left(\left\{L_{12}, M_{12}\right\}+\left\{N_{12}, N_{21}\right\}-\left\{N_{1}, N_{2}\right\}\right)$.

Comparing (6.19) with (6.17), we get the desired relation between the operators $\hat{\omega}$ and $a_{0}$

$\hat{\omega}=-a_{0}$.

The minus sign on the right-hand side is caused by the choice of the sign in (2.14). Let us make a comment with regard to an arbitrariness of a sign on the right-hand side of the relation (2.14). In the paper by Kemmer [5] the complete list of the algebraically inequivalent irreducible matrix representations of the DKP-algebra (3.1) for the case of an arbitrary number of elements $\beta_{\mu}$ is established and ranks of the representations is defined (see also Fujiwara [7]). An interesting feature for the case of an odd number of the $\beta_{\mu}$ is appearing so-called twin representations. In more exact terms, for any odd number $2 M+1$ of the elements $\beta_{\mu}$ there are two representations of the same rank as the highest ranked representation for $2 M$. As the matrix $\beta_{2 M+1}$ of the DKP-algebra with odd number of elements $\beta_{\mu}$ one takes matrix $\beta_{2 M+1}= \pm \omega$, where $\omega$ is defined by expression (3.2). Therefore, we have two alternatives that are algebraically inequivalent. In the case $M=2$, which is interesting from the physical point of view, in the situation when we have five elements $\beta_{\mu}$, the twin representations consist of the matrices of the representation of rank 10 for $\mu=1,2,3,4$ and the fifth matrix $\beta_{5}$ equals

$\beta_{5}= \pm \omega$

where now the matrix $\omega$ is defined by the expression (6.18). Thus, by virtue of the relation (6.20), uncertainty in a sign in (2.14) for the particular case $p=2$ and $M=2$ in view of (6.21) can be associated with the existence of the twin representations of rank 10 in the DKP-algebra.

Further, the relation (2.14) is true for an arbitrary order of parastatistics $p$ that hint at the fact that the twin representations could exist not only in the DKP-algebra but also in the higher-order algebras describing the particles with the spin greater than 1. The Bhabha-Madhavarao algebras for the spins $3 / 2$ and $2[41,56,57]$ could serve as an example of such algebras.

Now we can extend the algebra (4.4)-(4.6) for the paraFermi operators $a_{k}^{ \pm}$of order $p=2$ by incorporating the operator $a_{0}$. From the relations (A.1)-(A.5) in view of the relationship (6.20) we have

$a_{0}^{3}=a_{0}$,

$a_{0} a_{k}^{ \pm} a_{0}=0$,

$a_{0}^{2} a_{k}^{ \pm}+a_{k}^{ \pm} a_{0}^{2}=a_{k}^{ \pm}$, 
$a_{k}^{ \pm} a_{m}^{\mp} a_{0}+a_{0} a_{m}^{\mp} a_{k}^{ \pm}=2 \delta_{k m} a_{0}$,

$a_{k}^{ \pm} a_{m}^{ \pm} a_{0}+a_{0} a_{m}^{ \pm} a_{k}^{ \pm}=0$,

$a_{k}^{ \pm} a_{0} a_{m}^{ \pm}+a_{m}^{ \pm} a_{0} a_{k}^{ \pm}=0$,

$a_{k}^{ \pm} a_{0} a_{m}^{\mp}+a_{m}^{\mp} a_{0} a_{k}^{ \pm}=0$.

Nevertheless, we note that with respect to the operator relation (6.22c) in Sect. 20 as well as with respect to the matrix relation (A.3) we will have an important refinement.

\section{Matrix element of the operator $a_{0}$}

Given an explicit form of the operator $a_{0}$, Eq. (6.17), we can define its matrix element in the basis of the para-Fermi coherent states. We need this matrix element, in particular for determining the matrix element of the operator $\hat{A}$, which we present in two forms:

$\hat{A}=\alpha\left[\hat{I}-\left(\frac{i \sqrt{3}}{2}\right) a_{0}-\frac{3}{2} a_{0}^{2}\right]$,

$\hat{A}=\alpha\left[\hat{I}-\left(\frac{i \sqrt{3}}{2}\right) a_{0}-\frac{3}{2}\left(a_{0}\right)^{2}\right]$.

Here, from the original matrix definition (3.3) we have passed to the operator formulation and then, in accordance with (6.20), we have perform the replacement $\hat{\omega} \rightarrow-a_{0}$. We note that in the second expression (7.1b) we write exactly $\left(a_{0}\right)^{2} \equiv a_{0} \cdot a_{0}$ to distinguish it from the symbol $a_{0}^{2}$, which we keep for the notation of Geyer's operator (2.17), and which is used in the first expression (7.1a).

In Sect. 5 we have defined action of the operator $n_{k}=$ $N_{k}+1$ on the parafermion coherent state, Eq. (5.4). Hence it follows that

$N_{k}|\xi\rangle=\left(n_{k}-1\right)|\xi\rangle=\left(\frac{1}{2}\left[a_{k}^{+}, \xi_{k}\right]-1\right)|\xi\rangle$.

Let us consider action of the product $N_{2} N_{1}$ on the coherent state

$$
\begin{aligned}
N_{2} N_{1}|\xi\rangle= & \left(n_{2}-1\right)\left(\frac{1}{2}\left[a_{1}^{+}, \xi_{1}\right]-1\right)|\xi\rangle \\
= & \frac{1}{2}\left[n_{2},\left[a_{1}^{+}, \xi_{1}\right]\right]|\xi\rangle+\left(\frac{1}{2}\left[a_{1}^{+}, \xi_{1}\right]-1\right) \\
& \times\left(\frac{1}{2}\left[a_{2}^{+}, \xi_{2}\right]-1\right)|\xi\rangle .
\end{aligned}
$$

By using the commutation rules (2.4) and (B.3) it is not difficult to show that the double commutator on the right-hand side vanishes, and consequently we have

$$
\begin{aligned}
\left\{N_{1}, N_{2}\right\}|\xi\rangle= & \left\{\left(\frac{1}{2}\left[a_{1}^{+}, \xi_{1}\right]-1\right),\left(\frac{1}{2}\left[a_{2}^{+}, \xi_{2}\right]-1\right)\right\} \\
|\xi\rangle, &
\end{aligned}
$$

and matrix element for the anticommutator is

$$
\begin{aligned}
\left\langle\bar{\xi}^{\prime}\left|\left\{N_{1}, N_{2}\right\}\right| \xi\right\rangle= & 2\left(\frac{1}{2}\left[\bar{\xi}_{1}^{\prime}, \xi_{1}\right]-1\right)\left(\frac{1}{2}\left[\bar{\xi}_{2}^{\prime}, \xi_{2}\right]-1\right) \\
& \times\left\langle\bar{\xi}^{\prime} \mid \xi\right\rangle .
\end{aligned}
$$

Further we consider the operator expression $\left\{L_{12}, M_{12}\right\}$, which with the help of the second expression in (6.10), can be presented as

$$
\left\{L_{12}, M_{12}\right\}=2 L_{12} M_{12}+N_{1}+N_{2} .
$$

Recalling the definition of the operators $L_{12}$ and $M_{12}$, Eq. (6.9), taking into account the relation (7.2), it is easy to obtain the matrix element for the first anticommutator in (6.17)

$$
\begin{aligned}
& \left\langle\bar{\xi}^{\prime}\left|\left\{L_{12}, M_{12}\right\}\right| \xi\right\rangle \\
& =\left\{2\left(\frac{1}{2}\left[\bar{\xi}_{1}^{\prime}, \bar{\xi}_{2}^{\prime}\right]\right)\left(\frac{1}{2}\left[\xi_{1}, \xi_{2}\right]\right)+\left(\frac{1}{2}\left[\bar{\xi}_{1}^{\prime}, \xi_{1}\right]-1\right)\right. \\
& \left.\quad+\left(\frac{1}{2}\left[\bar{\xi}_{2}^{\prime}, \xi_{2}\right]-1\right)\right\}\left\langle\bar{\xi}^{\prime} \mid \xi\right\rangle .
\end{aligned}
$$

It remains for us only to define matrix element for the anticommutator $\left\{N_{12}, N_{21}\right\}$. Action of the generator $N_{21}$ on the coherent state is defined as

$N_{21}|\xi\rangle=\left[N_{21}, \mathrm{e}^{-\frac{1}{2}\left[\xi, a^{+}\right]}\right]|0\rangle+\mathrm{e}^{-\frac{1}{2}\left[\xi, a^{+}\right]} N_{21}|0\rangle$.

By virtue of the uniqueness conditions for the vacuum state (2.12) and (2.13) we have $N_{21}|0\rangle=0$ and therefore here, the last term vanishes. Taking into account the operator identity (5.3) and commutation rule (B.3), we obtain for the first term

$\left[N_{21}, \mathrm{e}^{-\frac{1}{2}\left[\xi, a^{+}\right]}\right]=\left[a_{2}^{+}, \xi_{1}\right] \mathrm{e}^{-\frac{1}{2}\left[\xi, a^{+}\right]}$

and thus we have

$N_{21}|\xi\rangle=\left(\frac{1}{2}\left[a_{2}^{+}, \xi_{1}\right]\right)|\xi\rangle$,
$N_{12}|\xi\rangle=\left(\frac{1}{2}\left[a_{1}^{+}, \xi_{2}\right]\right)|\xi\rangle$.

Hence we get

$$
\begin{aligned}
N_{12} N_{21}|\xi\rangle= & \frac{1}{2}\left[N_{12},\left[a_{2}^{+}, \xi_{1}\right]\right]|\xi\rangle \\
& +\left(\frac{1}{2}\left[a_{2}^{+}, \xi_{1}\right]\right) N_{12}|\xi\rangle .
\end{aligned}
$$

Here, for computing the double commutator we use the Jacobi identity and commutation rules (2.4), (B.3):

$$
\begin{aligned}
{\left[N_{12},\left[a_{2}^{+}, \xi_{1}\right]\right]=} & \frac{1}{2}\left[\left[a_{1}^{+}, a_{2}^{-}\right],\left[a_{2}^{+}, \xi_{1}\right]\right] \\
= & -\frac{1}{2}\left[a_{2}^{+},\left[\xi_{1},\left[a_{1}^{+}, a_{2}^{-}\right]\right]\right] \\
& -\frac{1}{2}\left[\xi_{1},\left[\left[a_{1}^{+}, a_{2}^{-}\right], a_{2}^{+}\right]\right] \\
= & -\left[\xi_{1}, a_{1}^{+}\right],
\end{aligned}
$$


and therefore

$$
\begin{aligned}
& N_{12} N_{21}|\xi\rangle \\
& =\left\{\left(\frac{1}{2}\left[a_{2}^{+}, \xi_{1}\right]\right)\left(\frac{1}{2}\left[a_{1}^{+}, \xi_{2}\right]\right)+\left(\frac{1}{2}\left[a_{1}^{+}, \xi_{1}\right]\right)\right\}|\xi\rangle .
\end{aligned}
$$

By analogy, we have

$$
\begin{aligned}
& N_{21} N_{12}|\xi\rangle \\
& =\left\{\left(\frac{1}{2}\left[a_{1}^{+}, \xi_{2}\right]\right)\left(\frac{1}{2}\left[a_{2}^{+}, \xi_{1}\right]\right)+\left(\frac{1}{2}\left[a_{2}^{+}, \xi_{2}\right]\right)\right\}|\xi\rangle
\end{aligned}
$$

Using the expressions obtained, we define the matrix element for the anticommutator $\left\{N_{12}, N_{21}\right\}$ :

$$
\begin{aligned}
& \left\langle\bar{\xi}^{\prime}\left|\left\{N_{12}, N_{21}\right\}\right| \xi\right\rangle \\
& =\left\{2\left(\frac{1}{2}\left[\bar{\xi}_{1}^{\prime}, \xi_{2}\right]\right)\left(\frac{1}{2}\left[\bar{\xi}_{2}^{\prime}, \xi_{1}\right]\right)+\left(\frac{1}{2}\left[\bar{\xi}_{1}^{\prime}, \xi_{1}\right]\right)\right. \\
& \left.\quad+\left(\frac{1}{2}\left[\bar{\xi}_{2}^{\prime}, \xi_{2}\right]\right)\right\}\left\langle\bar{\xi}^{\prime} \mid \xi\right\rangle .
\end{aligned}
$$

With allowance made for the expressions (7.3), (7.4) and (7.6), the desired matrix element of the operator $a_{0}$ takes the following form:

$$
\begin{aligned}
\left\langle\bar{\xi}^{\prime}\left|a_{0}\right| \xi\right\rangle=- & \frac{1}{2}\left\{\left(\frac{1}{2}\left[\bar{\xi}_{1}^{\prime}, \bar{\xi}_{2}^{\prime}\right]\right)\left(\frac{1}{2}\left[\xi_{1}, \xi_{2}\right]\right)\right. \\
& +\left(\frac{1}{2}\left[\bar{\xi}_{1}^{\prime}, \xi_{2}\right]\right)\left(\frac{1}{2}\left[\bar{\xi}_{2}^{\prime}, \xi_{1}\right]\right) \\
& -\left(\frac{1}{2}\left[\bar{\xi}_{1}^{\prime}, \xi_{1}\right]\right)\left(\frac{1}{2}\left[\bar{\xi}_{2}^{\prime}, \xi_{2}\right]\right) \\
& \left.+2\left(\frac{1}{2}\left[\bar{\xi}_{1}^{\prime}, \xi_{1}\right]+\frac{1}{2}\left[\bar{\xi}_{2}^{\prime}, \xi_{2}\right]-1\right)\right\}\left\langle\bar{\xi}^{\prime} \mid \xi\right\rangle
\end{aligned}
$$

This matrix element along with the matrix element for the operator $a_{0}^{2}$, Eq. (5.6), enables us to fully define the matrix element of the operator $\hat{A}$ as it was defined by the expression (7.1a).

In closing this section let us consider an indirect proof of the operator relation (6.22a) for our presentation (6.17). Matrix element of this relation can be presented as follows:

$$
\begin{aligned}
\left\langle\bar{\xi}^{\prime}\left|a_{0}\right| \xi\right\rangle= & \left\langle\bar{\xi}^{\prime}\left|a_{0}^{3}\right| \xi\right\rangle \\
= & \int\left\langle\bar{\xi}^{\prime}\left|a_{0}\right| \zeta\right\rangle\left\langle\bar{\zeta}\left|a_{0}^{2}\right| \xi\right\rangle \\
& \times \mathrm{e}^{-\frac{1}{2}[\bar{\zeta}, \zeta]}(d \zeta)_{2}(d \bar{\zeta})_{2} .
\end{aligned}
$$

Here, we have used the completeness relation (4.9). For the matrix element of the operator $a_{0}^{2}$ it is convenient to use the representation (5.13), then

$$
\begin{aligned}
& \left\langle\bar{\zeta}\left|a_{0}^{2}\right| \xi\right\rangle \mathrm{e}^{-\frac{1}{2}[\bar{\zeta}, \zeta]} \\
& \quad=\frac{1}{2}\left(\mathrm{e}^{\frac{1}{2}[\bar{\zeta}, \xi-\zeta]}+\mathrm{e}^{-\frac{1}{2}[\bar{\zeta}, \xi+\zeta]}\right) .
\end{aligned}
$$

Substituting the last expression into (7.8) and taking into account the definition of the para-Grassmann delta-function

$\int \mathrm{e}^{\frac{1}{2}[\bar{\zeta}, \xi-\zeta]}(d \bar{\zeta})_{2}=\delta(\xi-\zeta)$

where for the special case, $p=2$,

$$
\begin{aligned}
& \delta(\xi-\zeta) \equiv \prod_{j=1}^{2} \delta\left(\xi_{j}-\zeta_{j}\right), \\
& \delta\left(\xi_{j}-\zeta_{j}\right)=\frac{1}{i^{2} 2 !}\left(\xi_{j}-\zeta_{j}\right)^{2},
\end{aligned}
$$

instead of (7.8), we obtain

$\left\langle\bar{\xi}^{\prime}\left|a_{0}\right| \xi\right\rangle=\frac{1}{2}\left[\left\langle\bar{\xi}^{\prime}\left|a_{0}\right| \xi\right\rangle+\left\langle\bar{\xi}^{\prime}\left|a_{0}\right|-\xi\right\rangle\right]$.

Thereby, in order that the preceding expression turns into identity, the following equality must be true

$$
\left\langle\bar{\xi}^{\prime}\left|a_{0}\right|-\xi\right\rangle=\left\langle\bar{\xi}^{\prime}\left|a_{0}\right| \xi\right\rangle \text {. }
$$

By virtue of (7.7), the matrix element on the left-hand side has the following form:

$$
\begin{aligned}
\left\langle\bar{\xi}^{\prime}\left|a_{0}\right|-\xi\right\rangle=- & \frac{1}{2}\left\{\left(\frac{1}{2}\left[\bar{\xi}_{1}^{\prime}, \bar{\xi}_{2}^{\prime}\right]\right)\left(\frac{1}{2}\left[\xi_{1}, \xi_{2}\right]\right)\right. \\
& +\left(\frac{1}{2}\left[\bar{\xi}_{1}^{\prime}, \xi_{2}\right]\right)\left(\frac{1}{2}\left[\bar{\xi}_{2}^{\prime}, \xi_{1}\right]\right) \\
& -\left(\frac{1}{2}\left[\bar{\xi}_{1}^{\prime}, \xi_{1}\right]\right)\left(\frac{1}{2}\left[\bar{\xi}_{2}^{\prime}, \xi_{2}\right]\right) \\
& \left.-2\left(\frac{1}{2}\left[\bar{\xi}_{1}^{\prime}, \xi_{1}\right]+\frac{1}{2}\left[\bar{\xi}_{2}^{\prime}, \xi_{2}\right]+1\right)\right\} \\
& \times\left\langle\bar{\xi}^{\prime} \mid-\xi\right\rangle .
\end{aligned}
$$

We see that the sign of terms linear in commutators and in the overlap function, has changed. It is not at all obvious that the equality (7.11) will take place. The proof of (7.11) is given in the Appendix F.

\section{Connection between the operators $\hat{\omega}^{2}$ and $a_{0}^{2}$}

In Sect. 6 we have defined a connection between the operators $\hat{\omega}$ and $a_{0}$. Recall that the first of these operators arises naturally within the framework of the Duffin-Kemmer-Petiau formalism, whereas the second one enters into a generating set of the orthogonal group $S O(2 M+2)$. In this section, we would like to analyse independently a connection between the operators $\hat{\omega}^{2}$ and $a_{0}^{2}$. According to conclusions of Sect. 6 the operator $a_{0}^{2}$ introduced by Geyer [22] generally speaking, is not the square of the operator $a_{0}$ at least in the form given by expression (6.17).

In view of the general formula (67) from Harish-Chandra's paper [6] for $M=2$ we have the representation for the 
squared matrix $\omega^{2}$ :

$\omega^{2}=\frac{1}{4} \sum_{(\mathcal{P})} \beta_{\mu_{4}}^{2} \beta_{\mu_{2}}^{2}\left(1-\beta_{\mu_{3}}^{2}\right)\left(1-\beta_{\mu_{1}}^{2}\right)$,

where the indices $\mu_{1}, \mu_{2}, \mu_{3}$ and $\mu_{4}$ are all different and $\sum_{(\mathcal{P})}$ denotes a sum over all permutations $(1,2,3,4)$. Let us rewrite this expression in terms of Kemmer's matrices $[2,5]$

$\eta_{\mu}=2 \beta_{\mu}^{2}-1$

possessing the properties

$\eta_{\mu}^{2}=1, \quad \eta_{\mu} \eta_{\nu}=\eta_{\nu} \eta_{\mu}$

then

$\omega^{2}=\frac{1}{4^{3}} \sum_{(\mathcal{P})}\left(1+\eta_{\mu_{4}}\right)\left(1+\eta_{\mu_{2}}\right)\left(1-\eta_{\mu_{3}}\right)\left(1-\eta_{\mu_{1}}\right)$.

In view of (8.2), the sum on the right-hand side of (8.3) can be presented in the following form:

$$
\begin{aligned}
& 4 !\left(1+\eta_{5}\right)-8\left[\eta_{\mu_{1}} \eta_{\mu_{2}}+\eta_{\mu_{1}} \eta_{\mu_{3}}+\eta_{\mu_{1}} \eta_{\mu_{4}}\right. \\
& \left.\quad+\eta_{\mu_{2}} \eta_{\mu_{3}}+\eta_{\mu_{2}} \eta_{\mu_{4}}+\eta_{\mu_{3}} \eta_{\mu_{4}}\right] \equiv 4 !\left(1+\eta_{5}\right) \\
& \quad+4\left[4-\left(\eta_{\mu_{1}}+\eta_{\mu_{2}}+\eta_{\mu_{3}}+\eta_{\mu_{4}}\right)^{2}\right],
\end{aligned}
$$

where

$\eta_{5}=\eta_{1} \eta_{2} \eta_{3} \eta_{4}$

with the property

$\eta_{5} \eta_{\mu}=\eta_{\mu} \eta_{5}$

On the other hand, for the square of the sum $\eta_{\mu_{i}}$ in (8.4), by virtue of the definition (8.1), we obtain

$$
\begin{aligned}
\left(\sum_{i=1}^{4} \eta_{\mu_{i}}\right)^{2} & =\left(2 \sum_{i=1}^{4} \beta_{\mu_{i}}^{2}-4\right)^{2} \equiv(2 B-4)^{2} \\
& =\left(2-2 \omega^{2}\right)^{2}=4\left(1-\omega^{2}\right) .
\end{aligned}
$$

Here, we have used the definition of the matrix $B$ in Appendix A, the formula (A.6) for $M=2$, and the property (A.1).

Substituting (8.6) into (8.4) and further into (8.3), we obtain finally

$\omega^{2}=\frac{1}{2}\left(1+\eta_{5}\right)$.

It must be especially noted that we have not seen anywhere in literature such simple relation between the matrices $\omega$ and $\eta_{5}$. The most intriguing thing here is that two quantities entering into the relation have a rather different physical meaning. This difference has so clearly underlined in the paper by Krajcik and Nieto [58]. The matrix

$\omega=\frac{1}{4} \epsilon_{\mu \nu \lambda \sigma} \beta_{\mu} \beta_{\nu} \beta_{\lambda} \beta_{\sigma}$ plays a role of the "pseudoscalar operator" used in pseudoscalar coupling (in the Dirac theory analog of this matrix is $\left.(1 / 4 !) \epsilon_{\mu \nu \lambda \sigma} \gamma_{\mu} \gamma_{\nu} \gamma_{\lambda} \gamma_{\sigma}\right)$ while the matrix $\eta_{5}$, Eq. (8.5), plays a role of $C P T$ operator in the DKP theory (in the Dirac theory its analog is the matrix $\left.\gamma_{5}=\gamma_{1} \gamma_{2} \gamma_{3} \gamma_{4}\right)$. In the Dirac case the pseudoscalar and CPT operators are the same operator $\gamma_{5}$ by virtue of the purely algebraic peculiarities of the $\gamma$-matrices. However, in the DKP theory $\omega \neq \eta_{5}$, and the relation (8.7) shows us how these two different operators correlate among themselves.

Note, moreover, that relation (8.7) correctly reproduces formula (A.3) by virtue of the property $\left\{\eta_{5}, \beta_{\mu}\right\}=0$.

Now we turn to the consideration of the operator $a_{0}^{2}$ as it was defined by Geyer. Here, we give once again its explicit form

$a_{0}^{2}=1-\left[\left(N_{1}\right)^{2}+\left(N_{2}\right)^{2}\right]+2\left(N_{1}\right)^{2}\left(N_{2}\right)^{2}$,

where, we recall that $N_{k}=\frac{1}{2}\left[a_{k}^{+}, a_{k}^{-}\right]$. Let us rewrite the operator $a_{0}^{2}$ in terms of the operators $\widehat{\eta}_{\mu}$ as they follows from the matrix definition (8.1). By virtue of the representation (4.3), we have

$\left[a_{1}^{+}, a_{1}^{-}\right]=-2 i\left[\hat{\beta}_{1}, \hat{\beta_{2}}\right], \quad\left[a_{2}^{+}, a_{2}^{-}\right]=-2 i\left[\hat{\beta}_{3}, \hat{\beta}_{4}\right]$

and therefore, due to the DKP operator algebra (4.1) and the properties (8.2), we derive

$$
\begin{aligned}
\left(N_{1}\right)^{2} & =-\left[\hat{\beta}_{1}, \hat{\beta}_{2}\right]^{2}=\left(1-\hat{\beta}_{2}^{2}\right) \hat{\beta}_{1}^{2}+\left(1-\hat{\beta}_{1}^{2}\right) \hat{\beta}_{2}^{2} \\
& =\frac{1}{4}\left[\left(1-\widehat{\eta}_{2}\right)\left(1+\widehat{\eta}_{1}\right)+\left(1-\widehat{\eta}_{1}\right)\left(1+\widehat{\eta}_{2}\right)\right] \\
& =\frac{1}{2}\left(1-\widehat{\eta}_{1} \widehat{\eta}_{2}\right)
\end{aligned}
$$

and similar we get

$\left(N_{2}\right)^{2}=\frac{1}{2}\left(1-\widehat{\eta}_{3} \widehat{\eta}_{4}\right)$.

Substituting the obtained expressions (8.9) and (8.10) into (8.8), we define a connection between the operators $a_{0}^{2}$ and $\widehat{\eta}_{5}$ :

$a_{0}^{2}=\frac{1}{2}\left(1+\widehat{\eta}_{5}\right)$.

Comparing this expression with (8.7), we can conclude that

$\hat{\omega}^{2}=a_{0}^{2}$.

However, as it will be shown further, the operator relation (8.12) is true only in a some limited sense, and within the framework of our problem it is not correct and requires a principle improvement that will be done in Sect. 16. For ease of reading, hereafter the operator $\hat{\omega}^{2}$ will be named the Harish-Chandra operator and the operator $a_{0}^{2}$ will the Geyer operator. 


\section{Another representation for the Geyer operator $a_{0}^{2}$}

In this section we establish a connection between the operator $a_{0}^{2}$ and the parafermion number counter $(-1)^{n}$, where

$n=n_{1}+n_{2}$.

We begin our consideration with a reminder of how a similar connection arises for parastatistics $p=1$, i.e. in the Dirac theory and then we extend it to the case $p=2$. In usual Fermi statistics the operator $N_{k}=\frac{1}{2}\left[a_{k}^{+}, a_{k}^{-}\right]$satisfies the condition

$N_{k}^{2}=\frac{1}{4}, \quad k=1,2$,

by virtue of $\left\{a_{k}^{+}, a_{k}^{-}\right\}=1$ and $\left(a_{k}^{ \pm}\right)^{2}=0$. Further we introduce the operator

$$
\begin{aligned}
(-1)^{N_{1}+N_{2}} \equiv & \mathrm{e}^{i \pi\left(N_{1}+N_{2}\right)} \\
= & 1+i \pi\left(N_{1}+N_{2}\right) \\
& +\frac{1}{2 !}(i \pi)^{2}\left(N_{1}+N_{2}\right)^{2} \\
& +\frac{1}{3 !}(i \pi)^{3}\left(N_{1}+N_{2}\right)^{3}+\cdots .
\end{aligned}
$$

By straightforward calculation using the condition (9.2), it is not difficult to verify a validity of the relations

$$
\begin{aligned}
& \left(N_{1}+N_{2}\right)^{2 s+1}=N_{1}+N_{2}, \\
& \left(N_{1}+N_{2}\right)^{2 s}=\frac{1}{2}+2 N_{1} N_{2}, \quad s=0,1,2, \ldots
\end{aligned}
$$

and therefore we can write

$$
\begin{aligned}
(-1)^{N_{1}+N_{2}}= & 1+\left(i \pi+\frac{1}{3 !}(i \pi)^{3}+\cdots\right)\left(N_{1}+N_{2}\right) \\
& +\left(\frac{1}{2 !}(i \pi)^{2}+\frac{1}{4 !}(i \pi)^{4}+\cdots\right) \\
& \left(\frac{1}{2}+2 N_{1} N_{2}\right)=1+i \sin \pi\left(N_{1}+N_{2}\right) \\
& +(\cos \pi-1)\left(\frac{1}{2}+2 N_{1} N_{2}\right) \\
= & -4 N_{1} N_{2}=-2 a_{0} .
\end{aligned}
$$

Here, at the last step we have taken into account the relation (2.16). On the other hand, for $p=1$ we have

$2 a_{0} \equiv \hat{\gamma}_{5}=i^{2} \hat{\gamma}_{1} \hat{\gamma}_{2} \hat{\gamma}_{3} \hat{\gamma}_{4}$

If we introduce the fermion number operators for particles of the kind $k$

$n_{k}=N_{k}+\frac{1}{2}$,

then in terms of (9.1) we derive finally

$(-1)^{n}=2 a_{0}=\left(2 n_{1}-1\right)\left(2 n_{2}-1\right)$.
By doing so we reproduce the expression given in the paper by Dilkes, McKeon and Schubert [59] (see also D'Hoker and Gagné [60]).

Now we turn to the case of parastatistics $p=2$. Here, instead of the condition (9.2) we have

$N_{k}^{3}=N_{k}$.

As in the case of (9.4), we perform an analysis separately for even and odd powers of the sum $N_{1}+N_{2}$. By using (9.6) it is easy to obtain the explicit expressions for the first few even powers, which we write as follows:

$$
\begin{aligned}
& \left(N_{1}+N_{2}\right)^{2}=N_{1}^{2}+N_{2}^{2}-2 N_{1}^{2} N_{2}^{2}+2\left(N_{1} N_{2}+N_{1}^{2} N_{2}^{2}\right), \\
& \left(N_{1}+N_{2}\right)^{4}=N_{1}^{2}+N_{2}^{2}-2 N_{1}^{2} N_{2}^{2}+8\left(N_{1} N_{2}+N_{1}^{2} N_{2}^{2}\right), \\
& \left(N_{1}+N_{2}\right)^{6}=N_{1}^{2}+N_{2}^{2}-2 N_{1}^{2} N_{2}^{2}+32\left(N_{1} N_{2}+N_{1}^{2} N_{2}^{2}\right), \\
& \left(N_{1}+N_{2}\right)^{8}=N_{1}^{2}+N_{2}^{2}-2 N_{1}^{2} N_{2}^{2}+128\left(N_{1} N_{2}+N_{1}^{2} N_{2}^{2}\right) .
\end{aligned}
$$

It is not difficult to define a general form of the coefficient of the last term on the right-hand side if one notes that for the power 4 we have

$\sum_{k=0}^{1} C_{4}^{2 k+1}=C_{4}^{1}+C_{4}^{3}=8$,

for the power 6 we have

$\sum_{k=0}^{2} C_{6}^{2 k+1}=C_{6}^{1}+C_{6}^{3}=32$

etc. Here $C_{n}^{k}$ are the binomial coefficients. By this means we have

$$
\begin{aligned}
\left(N_{1}+N_{2}\right)^{2 n}= & N_{1}^{2}+N_{2}^{2}-2 N_{1}^{2} N_{2}^{2} \\
& +\left(\sum_{k=0}^{n-1} C_{2 n}^{2 k+1}\right)\left(N_{1} N_{2}+N_{1}^{2} N_{2}^{2}\right),
\end{aligned}
$$

where $n=1,2,3, \ldots$. By using formula for the sum of the binomial coefficients from Prudnikov et al. [61], finally we define

$$
\sum_{k=0}^{n-1} C_{2 n}^{2 k+1}=2^{2 n-1} .
$$

Now we consider odd powers. The first three nontrivial terms can be reduced to the following form:

$$
\begin{aligned}
& \left(N_{1}+N_{2}\right)^{3}=N_{1}+N_{2}+3\left(N_{1}^{2} N_{2}+N_{1} N_{2}^{2}\right), \\
& \left(N_{1}+N_{2}\right)^{5}=N_{1}+N_{2}+15\left(N_{1}^{2} N_{2}+N_{1} N_{2}^{2}\right), \\
& \left(N_{1}+N_{2}\right)^{7}=N_{1}+N_{2}+63\left(N_{1}^{2} N_{2}+N_{1} N_{2}^{2}\right) .
\end{aligned}
$$

The coefficient before the last term on the right-hand side for an arbitrary odd power $2 n+1$ equals a sum of all binomial coefficients (with the exception of the first and the last those) 
divided by 2 , i.e.

$\frac{1}{2}\left(\sum_{k=0}^{2 n+1} C_{2 n+1}^{k}-C_{2 n+1}^{0}-C_{2 n+1}^{2 n+1}\right)=2^{2 n}-1, \quad n \geq 1$.

It is easy to check that this formula correctly reproduces the coefficients in (9.9) and thus we get

$$
\begin{aligned}
\left(N_{1}+N_{2}\right)^{2 n+1}= & N_{1}+N_{2}+\left(2^{2 n}-1\right) \\
& \left(N_{1}^{2} N_{2}+N_{1} N_{2}^{2}\right) .
\end{aligned}
$$

We turn to the general expansion (9.3), which can be rewritten for the case under consideration as follows:

$$
\begin{aligned}
(-1)^{N_{1}+N_{2}}= & \mathrm{e}^{i \pi\left(N_{1}+N_{2}\right)} \\
\equiv & \cos \left[\pi\left(N_{1}+N_{2}\right)\right]+i \sin \left[\pi\left(N_{1}+N_{2}\right)\right] \\
= & \sum_{n=0}^{\infty} \frac{(-1)^{n}}{(2 n) !}\left[\pi\left(N_{1}+N_{2}\right)\right]^{2 n} \\
& +i \sum_{n=0}^{\infty} \frac{(-1)^{n}}{(2 n+1) !}\left[\pi\left(N_{1}+N_{2}\right)\right]^{2 n+1} .
\end{aligned}
$$

Let us substitute the above expressions (9.7), (9.8) and (9.10) into (9.11). Then for the sum of even powers we obtain

$$
\begin{aligned}
1+ & \left(N_{1}^{2}+N_{2}^{2}-2 N_{1}^{2} N_{2}^{2}\right)\left(\sum_{n=0}^{\infty} \frac{(-1)^{n}}{(2 n) !} \pi^{2 n}\right) \\
& +\left(N_{1} N_{2}+N_{1}^{2} N_{2}^{2}\right) \frac{1}{2}\left(\sum_{n=0}^{\infty} \frac{(-1)^{n}}{(2 n) !}(2 \pi)^{2 n}\right) \\
= & 1+\left(N_{1}^{2}+N_{2}^{2}-2 N_{1}^{2} N_{2}^{2}\right)(\cos \pi-1) \\
& +\left(N_{1} N_{2}+N_{1}^{2} N_{2}^{2}\right)(\cos 2 \pi-1) \\
= & 1-2\left(N_{1}^{2}+N_{2}^{2}-2 N_{1}^{2} N_{2}^{2}\right)
\end{aligned}
$$

and we get a similar expression for the sum of odd powers:

$$
\begin{aligned}
\left(N_{1}\right. & \left.+N_{2}\right)\left(\sum_{n=0}^{\infty} \frac{(-1)^{n}}{(2 n+1) !} \pi^{2 n+1}\right)+\left(N_{1}^{2} N_{2}+N_{1} N_{2}^{2}\right) \\
& \times\left\{\frac{1}{2}\left(\sum_{n=0}^{\infty} \frac{(-1)^{n}}{(2 n+1) !}(2 \pi)^{2 n+1}\right)\right. \\
& \left.-\left(\sum_{n=0}^{\infty} \frac{(-1)^{n}}{(2 n+1) !} \pi^{2 n+1}\right)\right\} \\
= & \left(N_{1}+N_{2}\right) \sin \pi+\left(N_{1}^{2} N_{2}+N_{1} N_{2}^{2}\right)\left(\frac{1}{2} \sin 2 \pi-\sin \pi\right) \\
= & 0 .
\end{aligned}
$$

Certainly, vanishing the contribution with odd powers is a consequence of evenness of the initial expression $(-1)^{N_{1}+N_{2}}$ with respect to the sum of operators $N_{1}+N_{2}$. Verifying this fact by a direct calculation serves to show consistency of the calculation scheme.
Thus, from (9.11) it follows that

$(-1)^{N_{1}+N_{2}}=1-2\left(N_{1}^{2}+N_{2}^{2}-2 N_{1}^{2} N_{2}^{2}\right)$.

The final step is to pass on the left-hand side of (9.12) to the parafermion number operators $n_{k}=N_{k}+1$ such that

$N_{1}+N_{2}=n_{1}+n_{2}-2 \equiv n-2$,

and for the right-hand side we recall the definition of the operator $a_{0}^{2}$, Eq. (8.8). As a result, instead of (9.12), we obtain

$(-1)^{n}=2 a_{0}^{2}-1$

or

$a_{0}^{2}=\frac{1}{2}\left[1+(-1)^{n}\right]$

This expression is an immediate generalization of formula (9.5) to the case of parastatistics $p=2$. It is interesting to note that the operator $a_{0}^{2}$ in the representation (9.13) coincides in its structure with the Gliozzi, Scherk and Olive operator [62] (the GSO projection), which projects onto states of even (para)fermion number.

A few consequences of the relation (9.13) can be obtained. Let us consider the matrix element of the operator $a_{0}^{2}$, then due to (9.13) we have

$$
\begin{aligned}
\left\langle\bar{\xi}^{\prime}\left|a_{0}^{2}\right| \xi\right\rangle & =\frac{1}{2}\left\{\left\langle\bar{\xi}^{\prime} \mid \xi\right\rangle+\left\langle\bar{\xi}^{\prime}\left|(-1)^{n}\right| \xi\right\rangle\right\} \\
& =\cosh \left(\frac{1}{2} \sum_{l}\left[\bar{\xi}_{l}^{\prime}, \xi_{l}\right]\right) .
\end{aligned}
$$

Here, we have taken into account the equality

$(-1)^{n}|\xi\rangle=|-\xi\rangle$

and therefore the overlap function is

$\left\langle\bar{\xi}^{\prime} \mid-\xi\right\rangle=\exp \left(-\frac{1}{2} \sum_{l}\left[\bar{\xi}_{l}^{\prime}, \xi_{l}\right]\right)$

Thus we reproduce the simple formula (5.13) obtained in Sect. 5 on the basis of completely different considerations.

Further, we consider the matrix element $\left\langle\bar{\xi}^{\prime}\left|\left[a_{0}^{2}, a_{k}^{+}\right]\right| \xi\right\rangle$. Taking into account that

$\left\{(-1)^{n}, a_{k}^{+}\right\}=0$ 
we get

$$
\begin{aligned}
\left\langle\bar{\xi}^{\prime}\left|\left[a_{0}^{2}, a_{k}^{+}\right]\right| \xi\right\rangle & =\frac{1}{2}\left\langle\bar{\xi}^{\prime}\left|\left[(-1)^{n}, a_{k}^{+}\right]\right| \xi\right\rangle \\
& =-\left\langle\bar{\xi}^{\prime}\left|a_{k}^{+}(-1)^{n}\right| \xi\right\rangle \\
& =-\bar{\xi}_{k}^{\prime}\left\langle\bar{\xi}^{\prime} \mid-\xi\right\rangle \\
& =-\bar{\xi}_{k}^{\prime} \mathrm{e}^{-\sum_{l}\left[\xi_{l}, \bar{\xi}_{l}^{\prime}\right]}\left\langle\bar{\xi}^{\prime} \mid \xi\right\rangle \\
& =-\frac{1}{2}\left(\frac{\partial}{\partial \xi_{k}} \mathrm{e}^{-\sum_{l}\left[\xi_{l}, \bar{\xi}_{l}^{\prime}\right]}\right)\left\langle\bar{\xi}^{\prime} \mid \xi\right\rangle \\
& =-\left(\frac{\partial \widetilde{\Omega}}{\partial \xi_{k}}\right)\left\langle\bar{\xi}^{\prime} \mid \xi\right\rangle .
\end{aligned}
$$

Here, we have used the differentiation rules (C.1), (C.2) and the definition of the function $\widetilde{\Omega}$ :

$\widetilde{\Omega}=\frac{1}{2}\left(1+\mathrm{e}^{-\sum_{l}\left[\xi_{l}, \bar{\xi}_{l}^{\prime}\right]}\right)$.

We would like to draw some parallel between Geyer [22] and Fukutome's [45] approaches. In the latter the problem of the construction of the algebra $\mathfrak{s o}(2 M+2)$ from the algebra $\mathfrak{s o}(2 M+1)$ was considered. For this purpose Fukutome has introduced the projectors

$P_{ \pm}=\frac{1}{2}\left[1 \pm(-1)^{n}\right]$

with the properties $P_{ \pm}^{2}=P_{ \pm}, P_{+} P_{-}=0$. By virtue of the relations (9.13) and (5.16), in our notations these projectors have the form:

$P_{+}=a_{0}^{2}, \quad P_{-}=1-a_{0}^{2}$.

The Lie algebra $\mathfrak{s o}(2 M+1)$ in [45] consists of the elements $\left\{a_{n}^{+}, a_{k}^{-}, E_{l}^{k}, E_{k l}, E^{k l}\right\}$, which correspond to the generators ${ }^{4}$ (E.1)

$E_{l}^{k}=N_{k l}, \quad E_{k l}=M_{k l}, \quad E^{k l}=L_{k l}, \quad E_{k}^{k}=N_{k}$.

For an extension of the algebra $\mathfrak{s o}(2 M+1)$ to the algebra $\mathfrak{s o}(2 M+2)$ Fukutome added new elements $\left\{E_{0}^{0}, E_{0}^{k}\right.$, $\left.E^{k 0}, E_{k 0}\right\}$, where

$$
\begin{array}{ll}
E_{0}^{0}=\frac{1}{2}\left(P_{-}+P_{+}\right)=\frac{1}{2}-a_{0}^{2}, & \\
E^{k}=a_{k}^{+} P_{-}=P_{+} a_{k}^{+}, & E^{0}=a_{k}^{-} P_{+}=P_{-} a_{k}^{-}, \\
E^{k 0}=-a_{k}^{+} P_{+}=-P_{-} a_{k}^{+}, & E^{0 k}=-E^{k 0}, \\
E_{k 0}=a_{k}^{-} P_{-}=P_{+} a_{k}^{-}, & E_{0 k}=-E_{k 0} .
\end{array}
$$

By this means, he constructed the algebra $\mathfrak{s o}(2 M+2)$ simply adding "by hand" new generating elements to the algebra $\mathfrak{s o}(2 M+1)$, whereas Geyer [22] immediately considered the

\footnotetext{
4 Note that in the construction of the Lie algebra $\mathfrak{s o}(2 M+1)$ Fukutome has used usual fermion creation and annihilation operators. In the case of para-Fermi statistics, we use the definition of the generator of algebra $\mathfrak{s o}(2 M+1)$ following the paper Bracken and Green [43].
}

algebra $\mathfrak{s o}(2 M+2)$, in which there is already a new element $\beta_{2 M+1} \equiv a_{0}$, Eq. (2.3), and this element in principle is not reduced to one of the elements (9.19). Therefore, in spite of some similarity of two approaches in the determination of the algebra $\mathfrak{s o}(2 M+2)$, one can state that they do not coincide literally.

The requirement of consistency of the representation (9.13) and the property $a_{0}^{3}=a_{0}$, Eq. (6.22a), lead us to the relations

$\left[(-1)^{n}, a_{0}\right]=0$

$(-1)^{n} a_{0}=a_{0}$.

The commutativity of the operators $(-1)^{n}$ and $a_{0}$ is a simple consequence of the representation (6.17), the property (9.16) and of the operator identity

$[A, B C]=\{A, B\} C-B\{A, C\}$.

A proof of the relation (9.20) is much more nontrivial. We consider it only in terms of the matrix elements.

In Sect. 6 we written out the rules of action of the operator $a_{0}$ on the state vectors. The operator $(-1)^{n}$ changes a sign for the states with the odd number of para-Fermi particles. However, as we see from the formulas (6.4) and (6.7), it is these states that vanish zero under the action of the operator $a_{0}$. In this sense the operator $a_{0}$ and the product $(-1)^{n} a_{0}$ are equivalent within the framework of the usual Fock space of the system under consideration. The situation changes drastically, when we use the para-Fermi coherent state $|\xi\rangle$ in the form of (4.7). The fact is that this definition of the coherent state in principle does not admit an expansion in the number basis

$\left(a_{i}^{+}\right)^{n}\left(a_{j}^{+}\right)^{m}|0\rangle, \quad i, j=1,2, \quad n, m \leq 2$.

Indeed, let us write the coherent state $|\xi\rangle$ in the form of an expansion in powers of $\sum_{l}\left[\xi_{l}, a_{l}^{+}\right]$:

$$
\begin{aligned}
|\xi\rangle= & \mathrm{e}^{-\frac{1}{2} \sum_{l}\left[\xi_{l}, a_{l}^{+}\right]}|0\rangle \\
= & |0\rangle+\left(-\frac{1}{2}\right) \sum_{l}\left[\xi_{l}, a_{l}^{+}\right]|0\rangle \\
& +\frac{1}{2 !}\left(-\frac{1}{2}\right)^{2}\left(\sum_{l}\left[\xi_{l}, a_{l}^{+}\right]\right)^{2}|0\rangle+\cdots .
\end{aligned}
$$

For the second term on the right-hand side we get

$\sum_{l}\left[\xi_{l}, a_{l}^{+}\right]|0\rangle=\sum_{l}\left(\xi_{l}|l\rangle-|l\rangle \xi_{l}\right)$,

where we have taken into account that

$\xi_{l}|0\rangle=|0\rangle \xi_{l}$ 
Here, the expansion in the basis (9.21) takes place. Further, for the third term in the expansion we have

$$
\begin{aligned}
& \sum_{l, l^{\prime}}\left[\xi_{l}, a_{l}^{+}\right]\left[\xi_{l^{\prime}}, a_{l^{\prime}}^{+}\right]|0\rangle=\sum_{l, l^{\prime}}\left[\xi_{l}, a_{l}^{+}\right]\left(\xi_{l^{\prime}}\left|l^{\prime}\right\rangle-\left|l^{\prime}\right\rangle \xi_{l^{\prime}}\right) \\
& =\sum_{l, l^{\prime}}\left(\xi_{l^{\prime}} \xi_{l}\left|l l^{\prime}\right\rangle-\xi_{l^{\prime}} a_{l}^{+} \xi_{l}\left|l^{\prime}\right\rangle-\xi_{l}\left|l l^{\prime}\right\rangle \xi_{l^{\prime}}-a_{l}^{+} \xi_{l}\left|l^{\prime}\right\rangle \xi_{l^{\prime}}\right) .
\end{aligned}
$$

We see that it is impossible to present the second and fourth terms in the above expression in the form (9.21) multiplied by para-Grassmann numbers as is the case of the first and third terms. This is a consequence of the fact that for parastatistics $p=2$ only trilinear relations of the form (B.6)-(B.8) are hold and therefore we have

$a_{k}^{ \pm} \xi_{n} \neq \xi_{n} a_{k}^{ \pm}$.

The equality takes place (with an accuracy of the factor $(-1)$ ) only for $p=1$, i.e. for the usual Fermi statistics and Grassmann numbers. By doing so, there is no the decomposition of $|\xi\rangle$ in the Fock space and ipso facto we come to a conception of the generalized state-vector space [26] including as well $\xi_{n}$ 's para-Grassmann numbers. We will discuss this question in more detail in the subsequent sections. Here, we only conclude that action of the operator $a_{0}$ on the coherent state $|\xi\rangle$ is not reduced to operations (6.3)-(6.8) and therefore a validity of the relation (9.20) is far from obvious in the generalized state-vector space. The proof of the relation (9.20) in basis of the coherent states comes down to the proof of the relation (7.11) (see Appendix F).

\section{Matrix elements of the products $\hat{A} a_{n}^{ \pm}$and of the commutators $\left[a_{0}, a_{n}^{ \pm}\right]$and $\left[a_{0}^{2}, a_{n}^{ \pm}\right]$}

In this section we calculate in an explicit form the matrix elements of the products $\hat{A} a_{n}^{ \pm}$and of the commutators $\left[a_{0}, a_{n}^{+}\right]$ and $\left[a_{0}^{2}, a_{n}^{+}\right]$. Let us consider the product $\hat{A} a_{n}^{-}$:

$\left\langle\bar{\xi}^{\prime}\left|\hat{A} a_{n}^{-}\right| \xi\right\rangle=\left\langle\bar{\xi}^{\prime}|\hat{A}| \xi\right\rangle \xi_{n}$.

A similar term with the creation operator $a_{n}^{+}$has somewhat a more complicated structure, since

$\hat{A} a_{n}^{+}=a_{n}^{+} \hat{A}+\left[\hat{A}, a_{n}^{+}\right]$

and therefore we can write

$$
\begin{aligned}
& \left\langle\bar{\xi}^{\prime}\left|\hat{A} a_{n}^{+}\right| \xi\right\rangle \\
& =\bar{\xi}_{n}^{\prime}\left\langle\bar{\xi}^{\prime}|\hat{A}| \xi\right\rangle-\beta\left\langle\bar{\xi}^{\prime}\left|\left[a_{0}, a_{n}^{+}\right]\right| \xi\right\rangle \\
& +\gamma\left\langle\bar{\xi}^{\prime}\left|\left[a_{0}^{2}, a_{n}^{+}\right]\right| \xi\right\rangle .
\end{aligned}
$$

Here, we have taken into account the representation of the operator $\hat{A}$ in the first form (7.1a). Therefore, we are confronted by the task of deriving matrix elements of the commutators $\left[a_{0}, a_{n}^{+}\right]$and $\left[a_{0}^{2}, a_{n}^{+}\right]$. Let us consider the first of them.
By virtue of the representation of the operator $a_{0}$, Eq. (6.17), we have

$$
\begin{aligned}
{\left[a_{0}, a_{n}^{+}\right] } \\
=-\frac{1}{4}\left(\left[\left\{L_{12}, M_{12}\right\}, a_{n}^{+}\right]+\left[\left\{N_{12}, N_{21}\right\}, a_{n}^{+}\right]\right. \\
\left.\quad-2\left[N_{1} N_{2}, a_{n}^{+}\right]\right) .
\end{aligned}
$$

By using the operator identity

$$
[\{A, B\}, C]=\{A,[B, C]\}+\{B,[A, C]\}
$$

and commutation rules (6.15), it is not difficult to obtain a more simple form of the commutators on the right-hand side (10.3). We write them in two representations: the first of them is

$$
\begin{aligned}
& {\left[\left\{L_{12}, M_{12}\right\}, a_{n}^{+}\right]=\delta_{n 2}\left\{L_{12}, a_{1}^{-}\right\}-\delta_{n 1}\left\{L_{12}, a_{2}^{-}\right\},} \\
& {\left[\left\{N_{12}, N_{21}\right\}, a_{n}^{+}\right]=\delta_{n 2}\left\{N_{21}, a_{1}^{+}\right\}+\delta_{n 1}\left\{N_{12}, a_{2}^{+}\right\},} \\
& {\left[N_{1} N_{2}, a_{n}^{+}\right]=\delta_{n 2} a_{2}^{+} N_{1}+\delta_{n 1} a_{1}^{+} N_{2}}
\end{aligned}
$$

and the second one is

$$
\begin{aligned}
{\left[\left\{L_{12}, M_{12}\right\}, a_{n}^{+}\right]=} & 2 L_{12}\left(\delta_{n 2} a_{1}^{-}-\delta_{n 1} a_{2}^{-}\right) \\
& +\left(\delta_{n 2} a_{2}^{+}+\delta_{n 1} a_{1}^{+}\right), \\
{\left[\left\{N_{12}, N_{21}\right\}, a_{n}^{+}\right]=} & 2 \delta_{n 2} a_{1}^{+} N_{21}+2 \delta_{n 1} a_{2}^{+} N_{12} \\
& +\left(\delta_{n 2} a_{2}^{+}+\delta_{n 1} a_{1}^{+}\right), \\
{\left[N_{1} N_{2}, a_{n}^{+}\right]=} & \delta_{n 2} a_{2}^{+} N_{1}+\delta_{n 1} a_{1}^{+} N_{2} .
\end{aligned}
$$

In Appendix $\mathrm{G}$ we use the first representation in the proof of turning into identity the commutation relations including the operator $a_{0}$, Eqs. (2.7)-(2.11). The second one is more convenient for deriving the required matrix element $\left\langle\bar{\xi}^{\prime}\left|\left[a_{0}, a_{n}^{+}\right]\right| \xi\right\rangle$ (and also $\left\langle\bar{\xi}^{\prime}\left|\hat{A}\left[a_{0}, a_{n}^{+}\right]\right| \xi\right\rangle$, see the next section).

We need the matrix elements of the generators $L_{12}$, $M_{12}, \ldots$, which can be easily obtained from their definitions (6.9):

$$
\begin{aligned}
& \left\langle\bar{\xi}^{\prime}\left|L_{12}\right| \xi\right\rangle=\left(\frac{1}{2}\left[\bar{\xi}_{1}^{\prime}, \bar{\xi}_{2}^{\prime}\right]\right)\left\langle\bar{\xi}^{\prime} \mid \xi\right\rangle, \\
& \left\langle\bar{\xi}^{\prime}\left|M_{12}\right| \xi\right\rangle=\left(\frac{1}{2}\left[\xi_{1}, \xi_{2}\right]\right)\left\langle\bar{\xi}^{\prime} \mid \xi\right\rangle, \\
& \left\langle\bar{\xi}^{\prime}\left|N_{12}\right| \xi\right\rangle=\left(\frac{1}{2}\left[\bar{\xi}_{1}^{\prime}, \xi_{2}\right]\right)\left\langle\bar{\xi}^{\prime} \mid \xi\right\rangle, \\
& \left\langle\bar{\xi}^{\prime}\left|N_{21}\right| \xi\right\rangle=\left(\frac{1}{2}\left[\bar{\xi}_{2}^{\prime}, \xi_{1}\right]\right)\left\langle\bar{\xi}^{\prime} \mid \xi\right\rangle, \\
& \left\langle\bar{\xi}^{\prime}\left|N_{1}\right| \xi\right\rangle=\left\{\left(\frac{1}{2}\left[\bar{\xi}_{1}^{\prime}, \xi_{1}\right]\right)-1\right\}\left\langle\bar{\xi}^{\prime} \mid \xi\right\rangle, \\
& \left\langle\bar{\xi}^{\prime}\left|N_{2}\right| \xi\right\rangle=\left\{\left(\frac{1}{2}\left[\bar{\xi}_{2}^{\prime}, \xi_{2}\right]\right)-1\right\}\left\langle\bar{\xi}^{\prime} \mid \xi\right\rangle .
\end{aligned}
$$

Substituting (10.6) into (10.3) and taking into account (10.7), we obtain the desired matrix element 


$$
\begin{aligned}
\left\langle\bar{\xi}^{\prime}\right|[ & \left.a_{0}, a_{n}^{+}\right]|\xi\rangle \\
= & -\frac{1}{2}\left\{\left(\frac{1}{2}\left[\bar{\xi}_{1}^{\prime}, \bar{\xi}_{2}^{\prime}\right]\right)\left(\delta_{n 2} \xi_{1}-\delta_{n 1} \xi_{2}\right)\right. \\
+ & \delta_{n 1} \bar{\xi}_{2}^{\prime}\left(\frac{1}{2}\left[\bar{\xi}_{1}^{\prime}, \xi_{2}\right]\right)+\delta_{n 2} \bar{\xi}_{1}^{\prime}\left(\frac{1}{2}\left[\bar{\xi}_{2}^{\prime}, \xi_{1}\right]\right) \\
& -\delta_{n 2} \bar{\xi}_{2}^{\prime}\left(\frac{1}{2}\left[\bar{\xi}_{1}^{\prime}, \xi_{1}\right]\right)-\delta_{n 1} \bar{\xi}_{1}^{\prime}\left(\frac{1}{2}\left[\bar{\xi}_{2}^{\prime}, \xi_{2}\right]\right) \\
& \left.+2\left(\delta_{n 2} \bar{\xi}_{2}^{\prime}+\delta_{n 1} \bar{\xi}_{1}^{\prime}\right)\right\}\left\langle\bar{\xi}^{\prime} \mid \xi\right\rangle .
\end{aligned}
$$

The expression can be presented in a more compact and visual form. For this purpose we write the matrix element of operator $a_{0}$ in the following form:

$$
\left\langle\bar{\xi}^{\prime}\left|a_{0}\right| \xi\right\rangle=\Omega\left\langle\bar{\xi}^{\prime} \mid \xi\right\rangle,
$$

where in accordance with (7.7) we have

$$
\begin{aligned}
\Omega \equiv & \Omega\left(\bar{\xi}^{\prime}, \xi\right) \\
= & -\frac{1}{2}\left\{\left(\frac{1}{2}\left[\bar{\xi}_{1}^{\prime}, \bar{\xi}_{2}^{\prime}\right]\right)\left(\frac{1}{2}\left[\xi_{1}, \xi_{2}\right]\right)\right. \\
& +\left(\frac{1}{2}\left[\bar{\xi}_{1}^{\prime}, \xi_{2}\right]\right)\left(\frac{1}{2}\left[\bar{\xi}_{2}^{\prime}, \xi_{1}\right]\right) \\
& -\left(\frac{1}{2}\left[\bar{\xi}_{1}^{\prime}, \xi_{1}\right]\right)\left(\frac{1}{2}\left[\bar{\xi}_{2}^{\prime}, \xi_{2}\right]\right) \\
& \left.+2\left(\frac{1}{2}\left[\bar{\xi}_{1}^{\prime}, \xi_{1}\right]+\frac{1}{2}\left[\bar{\xi}_{2}^{\prime}, \xi_{2}\right]-1\right)\right\} .
\end{aligned}
$$

Let us take the derivative of the function $\Omega$ with respect to $\xi_{n}$ by making use of the rules of differentiation (C.1) and (C.2)

$$
\begin{aligned}
\frac{\partial \Omega}{\partial \xi_{n}}=- & \frac{1}{2}\left\{\left(\frac{1}{2}\left[\bar{\xi}_{1}^{\prime}, \bar{\xi}_{2}^{\prime}\right]\right)\left(\delta_{n 1} \xi_{2}-\delta_{n 2} \xi_{1}\right)\right. \\
& -\frac{1}{2} \bar{\xi}_{2}^{\prime}\left[\bar{\xi}_{1}^{\prime},\left(\delta_{n 1} \xi_{2}-\delta_{n 2} \xi_{1}\right)\right] \\
& +\frac{1}{2} \bar{\xi}_{1}^{\prime}\left[\bar{\xi}_{2}^{\prime},\left(\delta_{n 1} \xi_{2}-\delta_{n 2} \xi_{1}\right)\right] \\
& \left.-2\left(\delta_{n 1} \bar{\xi}_{1}^{\prime}+\delta_{n 2} \bar{\xi}_{2}^{\prime}\right)\right\} .
\end{aligned}
$$

Comparing the last expression with (10.8), we obtain that

$\left\langle\bar{\xi}^{\prime}\left|\left[a_{0}, a_{n}^{+}\right]\right| \xi\right\rangle=-\left(\frac{\partial \Omega}{\partial \xi_{n}}\right)\left\langle\bar{\xi}^{\prime} \mid \xi\right\rangle$.

Similar reasoning for the commutator $\left[a_{0}, a_{n}^{-}\right]$leads us to the representation of the corresponding matrix element

$\left\langle\bar{\xi}^{\prime}\left|\left[a_{0}, a_{n}^{-}\right]\right| \xi\right\rangle=-\left(\frac{\partial \Omega}{\partial \bar{\xi}_{n}^{\prime}}\right)\left\langle\bar{\xi}^{\prime} \mid \xi\right\rangle$,

where

$$
\begin{aligned}
\frac{\partial \Omega}{\partial \bar{\xi}_{n}^{\prime}}=- & \frac{1}{2}\left\{\left(\delta_{n 1} \bar{\xi}_{2}^{\prime}-\delta_{n 2} \bar{\xi}_{1}^{\prime}\right)\left(\frac{1}{2}\left[\xi_{1}, \xi_{2}\right]\right)\right. \\
& -\frac{1}{2}\left[\left(\delta_{n 1} \bar{\xi}_{2}^{\prime}-\delta_{n 2} \bar{\xi}_{1}^{\prime}\right), \xi_{2}\right] \xi_{1}
\end{aligned}
$$

$$
\begin{aligned}
& +\frac{1}{2}\left[\left(\delta_{n 1} \bar{\xi}_{2}^{\prime}-\delta_{n 2} \bar{\xi}_{1}^{\prime}\right), \xi_{1}\right] \xi_{2} \\
& \left.+2\left(\delta_{n 1} \xi_{1}+\delta_{n 2} \xi_{2}\right)\right\} .
\end{aligned}
$$

Now we turn to an analysis of the matrix element $\left\langle\bar{\xi}^{\prime}\left|\left[a_{0}^{2}, a_{n}^{+}\right]\right| \xi\right\rangle$. By virtue of Geyer's representation (8.8) we have the starting expression

$$
\begin{aligned}
\left\langle\bar{\xi}^{\prime}\left|\left[a_{0}^{2}, a_{n}^{+}\right]\right| \xi\right\rangle= & -\left\langle\bar{\xi}^{\prime}\left|\left[N_{1}^{2}, a_{n}^{+}\right]\right| \xi\right\rangle-\left\langle\bar{\xi}^{\prime}\left|\left[N_{2}^{2}, a_{n}^{+}\right]\right| \xi\right\rangle \\
& +2\left\langle\bar{\xi}^{\prime}\left|\left[N_{1}^{2} N_{2}^{2}, a_{n}^{+}\right]\right| \xi\right\rangle .
\end{aligned}
$$

By using the last two formulas in the commutation rules (6.15), we obtain

$\left[N_{k}^{2}, a_{n}^{+}\right]=\delta_{k n} a_{n}^{+}+2 \delta_{k n} a_{n}^{+} N_{k}$.

Matrix element of this commutator equals

$$
\begin{aligned}
& \left\langle\bar{\xi}^{\prime}\left|\left[N_{k}^{2}, a_{n}^{+}\right]\right| \xi\right\rangle \\
& =\left\{\delta_{k n} \bar{\xi}_{n}^{\prime}+2 \delta_{k n} \bar{\xi}_{n}^{\prime}\left(\frac{1}{2}\left[\bar{\xi}_{k}^{\prime}, \xi_{k}\right]-1\right)\right\}\left\langle\bar{\xi}^{\prime} \mid \xi\right\rangle .
\end{aligned}
$$

Further, the commutator with the product $N_{1}^{2} N_{2}^{2}$ in (10.15) has the form

$$
\begin{aligned}
{\left[N_{1}^{2} N_{2}^{2}, a_{n}^{+}\right]=} & \delta_{n 2} a_{2}^{+}\left(N_{1}^{2}+2 N_{2} N_{1}^{2}\right) \\
& +\delta_{n 1} a_{1}^{+}\left(N_{2}^{2}+2 N_{1} N_{2}^{2}\right) \\
& +\delta_{n 1} \delta_{n 2} a_{n}^{+}\left(1+2 N_{1}\right)\left(1+2 N_{2}\right) .
\end{aligned}
$$

The last term here vanishes for $M=2$. We need a matrix element of operator $N_{k}^{2}$. It can easily be obtained from the formulas (5.4) and (5.5) with regard to the definition (5.1)

$$
\begin{aligned}
\left\langle\bar{\xi}^{\prime}\left|N_{k}^{2}\right| \xi\right\rangle= & \left\{\left(\frac{1}{2}\left[\bar{\xi}_{k}^{\prime}, \xi_{k}\right]\right)^{2}-\left(\frac{1}{2}\left[\bar{\xi}_{k}^{\prime}, \xi_{k}\right]\right)+1\right\} \\
& \times\left\langle\bar{\xi}^{\prime} \mid \xi\right\rangle .
\end{aligned}
$$

As a consequence of commutativity of the operators $N_{1}$ and $N_{2}$ we have

$$
\begin{aligned}
\left\langle\bar{\xi}^{\prime}\left|N_{2} N_{1}^{2}\right| \xi\right\rangle= & \left(\frac{1}{2}\left[\bar{\xi}_{2}^{\prime}, \xi_{2}\right]-1\right) \\
& \times\left\{\left(\frac{1}{2}\left[\bar{\xi}_{1}^{\prime}, \xi_{1}\right]\right)^{2}-\left(\frac{1}{2}\left[\bar{\xi}_{1}^{\prime}, \xi_{1}\right]\right)+1\right\} \\
& \times\left\langle\bar{\xi}^{\prime} \mid \xi\right\rangle
\end{aligned}
$$

and a similar expression for the product $N_{1} N_{2}^{2}$ with the replacement $1 \rightleftarrows 2$. Substituting the obtained expressions into (10.15), we derive the explicit form of the desired matrix 
element

$$
\begin{aligned}
\left\langle\bar{\xi}^{\prime}\right| & {\left[a_{0}^{2}, a_{n}^{+}\right]|\xi\rangle } \\
= & \bar{\xi}_{n}^{\prime}\left[\delta _ { n 1 } \left\{-1-2\left(\frac{1}{2}\left[\bar{\xi}_{1}^{\prime}, \xi_{1}\right]-1\right)\right.\right. \\
& +2\left[\left(\frac{1}{2}\left[\bar{\xi}_{2}^{\prime}, \xi_{2}\right]\right)^{2}-\left(\frac{1}{2}\left[\bar{\xi}_{2}^{\prime}, \xi_{2}\right]\right)+1\right] \\
& +4\left(\frac{1}{2}\left[\bar{\xi}_{1}^{\prime}, \xi_{1}\right]-1\right)\left[\left(\frac{1}{2}\left[\bar{\xi}_{2}^{\prime}, \xi_{2}\right]\right)^{2}\right. \\
& \left.\left.\left.-\left(\frac{1}{2}\left[\bar{\xi}_{2}^{\prime}, \xi_{2}\right]\right)+1\right]\right\}+(1 \rightleftarrows 2)\right] \\
& \times\left\langle\bar{\xi}^{\prime} \mid \xi\right\rangle .
\end{aligned}
$$

This expression can be given in a more visual form if one takes into account the fact that

$$
\begin{aligned}
& \left(\frac{1}{2}\left[\bar{\xi}_{k}^{\prime}, \xi_{k}\right]\right)^{2}-\left(\frac{1}{2}\left[\bar{\xi}_{k}^{\prime}, \xi_{k}\right]\right)+1 \equiv \frac{1}{\left(\frac{1}{2}\left[\bar{\xi}_{k}^{\prime}, \xi_{k}\right]\right)+1}, \\
& k=1,2 .
\end{aligned}
$$

The relation holds by virtue of algebra (5.8). Then by using the notations $x$ and $y$ introduced in Sect. 5, Eq. (5.7), instead of (10.17), we have

$$
\begin{aligned}
\left\langle\bar{\xi}^{\prime}\right| & {\left[a_{0}^{2}, a_{n}^{+}\right]|\xi\rangle } \\
= & \bar{\xi}_{n}^{\prime}\left[\delta_{n 1}\left\{-1+2\left[(1-x)+\frac{1}{1+y}-2 \frac{1-x}{1+y}\right]\right\}\right. \\
& +\delta_{n 2}\left\{-1+2\left[(1-y)+\frac{1}{1+x}-2 \frac{1-y}{1+x}\right]\right\} \\
& \times\left\langle\bar{\xi}^{\prime} \mid \xi\right\rangle .
\end{aligned}
$$

We note also that the matrix element $\left\langle\bar{\xi}^{\prime}\left|a_{0}^{2}\right| \xi\right\rangle$ defined by expression (5.6), can be given in a similar form

$$
\begin{aligned}
& \left\langle\bar{\xi}^{\prime}\left|a_{0}^{2}\right| \xi\right\rangle \\
& =\left(1-\left[\frac{1}{1+x}+\frac{1}{1+y}\right]+2 \frac{1}{(1+x)(1+y)}\right)\left\langle\bar{\xi}^{\prime} \mid \xi\right\rangle \\
& \equiv \frac{1}{2}\left[1+\frac{(1-x)(1-y)}{(1+x)(1+y)}\right]\left\langle\bar{\xi}^{\prime} \mid \xi\right\rangle .
\end{aligned}
$$

By straightforward calculation one can easily check the correctness of the following relations:

$\mathrm{e}^{2 x}=\frac{(1+x)}{(1-x)}, \quad \mathrm{e}^{2 y}=\frac{(1+y)}{(1-y)}$.

Taking into account these relations and the form of the overlap function (5.12), we get, instead of (10.18),

$\left\langle\bar{\xi}^{\prime}\left|a_{0}^{2}\right| \xi\right\rangle=\frac{1}{2}\left(1+\mathrm{e}^{-2(x+y)}\right) \mathrm{e}^{x+y}=\cosh (x+y)$.

By doing so, we have shown by the third way a correctness of the expression (5.13) (the second way was considered in the previous section).
Let us return to the expression (10.17). We present it in the form similar to (10.12) for the matrix element of commutator the $\left[a_{0}, a_{n}^{+}\right]$. For this purpose, we write out the matrix element of the operator $a_{0}^{2}$ as follows:

$\left\langle\bar{\xi}^{\prime}\left|a_{0}^{2}\right| \xi\right\rangle=\widetilde{\Omega}\left\langle\bar{\xi}^{\prime} \mid \xi\right\rangle$,

where in accordance with (5.6), we have

$$
\begin{aligned}
\widetilde{\Omega}= & \widetilde{\Omega}\left(\bar{\xi}^{\prime}, \xi\right) \\
= & 1-\sum_{k=1}^{2}\left[\left(\frac{1}{2}\left[\bar{\xi}_{k}^{\prime}, \xi_{k}\right]\right)^{2}-\frac{1}{2}\left[\bar{\xi}_{k}^{\prime}, \xi_{k}\right]+1\right] \\
& +2 \prod_{k=1}^{2}\left[\left(\frac{1}{2}\left[\bar{\xi}_{k}^{\prime}, \xi_{k}\right]\right)^{2}-\frac{1}{2}\left[\bar{\xi}_{k}^{\prime}, \xi_{k}\right]+1\right] .
\end{aligned}
$$

By a direct calculation, using the formulas of differentiation (C.1) and (C.2), it is easy to verify that the following relation $\left\langle\bar{\xi}^{\prime}\left|\left[a_{0}^{2}, a_{n}^{+}\right]\right| \xi\right\rangle=-\left(\frac{\partial \widetilde{\Omega}}{\partial \xi_{n}}\right)\left\langle\bar{\xi}^{\prime} \mid \xi\right\rangle$

is true. In this way we reproduce the result (9.17). The same reasoning leads to

$\left\langle\bar{\xi}^{\prime}\left|\left[a_{0}^{2}, a_{n}^{-}\right]\right| \xi\right\rangle=-\left(\frac{\partial \widetilde{\Omega}}{\partial \bar{\xi}_{n}^{\prime}}\right)\left\langle\bar{\xi}^{\prime} \mid \xi\right\rangle$.

We write the matrix element of the operator $\hat{A}$ in a form similar to the form of expressions (10.9) and (10.19):

$\left\langle\bar{\xi}^{\prime}|\hat{A}| \xi\right\rangle=\mathcal{A}\left\langle\bar{\xi}^{\prime} \mid \xi\right\rangle$.

An explicit form of the function $\mathcal{A}=\mathcal{A}\left(\bar{\xi}^{\prime}, \xi\right)$ can be written out based on the operator expression (7.1) and with allowance made for (10.9), (10.10) and (10.19), (10.20). However we will postpone it until the Sect. 15, where we consider in detail a question of a connection between the operator $a_{0}^{2}$ defined by the expression (8.8) and the square of the operator $a_{0}$ (i.e. $\left.\left(a_{0}\right)^{2} \equiv a_{0} \cdot a_{0}\right)$ defined by the expression (6.17). Let us recall that the square of the operator $a_{0}$ enters into the second representation of the operator $\hat{A}$, Eq. (7.1b).

For the remaining two terms in (10.2) we use the representations (10.12) and (10.21), correspondingly. As a result, instead of (10.2), we have

$\left\langle\bar{\xi}^{\prime}\left|\hat{A} a_{n}^{+}\right| \xi\right\rangle=\left(\bar{\xi}_{n}^{\prime} \mathcal{A}-\frac{\partial \mathcal{A}}{\partial \xi_{n}}\right)\left\langle\bar{\xi}^{\prime} \mid \xi\right\rangle$.

Follow the same procedure, we can write out the matrix element for the product $a_{n}^{-} \hat{A}$

$\left\langle\bar{\xi}^{\prime}\left|a_{n}^{-} \hat{A}\right| \xi\right\rangle=\left(\xi_{n} \mathcal{A}-\frac{\partial \mathcal{A}}{\partial \bar{\xi}_{n}^{\prime}}\right)\left\langle\bar{\xi}^{\prime} \mid \xi\right\rangle$.

The last two expressions will be used in the following section. In the accepted notations the matrix element (10.1) is rewritten in the form

$\left\langle\bar{\xi}^{\prime}\left|\hat{A} a_{n}^{-}\right| \xi\right\rangle=\xi_{n} \mathcal{A}\left\langle\bar{\xi}^{\prime} \mid \xi\right\rangle$. 
The absence of the term with derivative in the last expression in comparison with (10.25) is connected with the fact that operators $\hat{A}$ and $a_{n}^{-}$are not commutative.

\section{Matrix elements of the product $\hat{A}\left[a_{0}, a_{n}^{ \pm}\right]$}

Now we proceed to the calculation of the matrix elements of the product $\hat{A}\left[a_{0}, a_{n}^{ \pm}\right]$. We need the following representations for the commutators $\left[a_{0}, a_{n}^{-}\right]$and $\left[a_{0}, a_{n}^{+}\right]$:

$$
\begin{aligned}
& {\left[a_{0}, a_{n}^{-}\right]} \\
& \quad=-\frac{1}{2}\left\{\left(\delta_{n 2} a_{1}^{+}-\delta_{n 1} a_{2}^{+}\right) M_{12}-\left(\delta_{n 1} N_{21} a_{2}^{-}+\delta_{n 2} N_{12} a_{1}^{-}\right)\right. \\
& \left.\quad+\left(\delta_{n 2} N_{1} a_{2}^{-}+\delta_{n 1} N_{2} a_{1}^{-}\right)-\left(\delta_{n 2} a_{2}^{-}+\delta_{n 1} a_{1}^{-}\right)\right\}, \\
& {\left[a_{0}, a_{n}^{+}\right]} \\
& \quad=-\frac{1}{2}\left\{L_{12}\left(\delta_{n 2} a_{1}^{-}-\delta_{n 1} a_{2}^{-}\right)+\left(\delta_{n 2} a_{1}^{+} N_{21}+\delta_{n 1} a_{2}^{+} N_{12}\right)\right. \\
& \left.\quad-\left(\delta_{n 2} a_{2}^{+} N_{1}+\delta_{n 1} a_{1}^{+} N_{2}\right)+\left(\delta_{n 2} a_{2}^{+}+\delta_{n 1} a_{1}^{+}\right)\right\} .
\end{aligned}
$$

A proof of the second representation was given in the previous section, Eqs. (10.3) and (10.6), the first one is proved in a similar way. Further we consider action of the commutator (11.1) on the parafermion coherent state

$$
\begin{aligned}
{\left[a_{0}, a_{n}^{-}\right]|\xi\rangle=} & -\frac{1}{2}\left\{\left(\delta_{n 2} a_{1}^{+}-\delta_{n 1} a_{2}^{+}\right)\left(\frac{1}{2}\left[\xi_{1}, \xi_{2}\right]\right)\right. \\
& -\left(\delta_{n 1} N_{21} \xi_{2}+\delta_{n 2} N_{12} \xi_{1}\right) \\
& \left.+\left(\delta_{n 2} N_{1} \xi_{2}+\delta_{n 1} N_{2} \xi_{1}\right)-\left(\delta_{n 2} \xi_{2}+\delta_{n 1} \xi_{1}\right)\right\} \\
& \times|\xi\rangle .
\end{aligned}
$$

Action of the generators $N_{1}, N_{2}, N_{12}$ and $N_{21}$ on the coherent state was defined by us in Sect. 7, Eqs. (7.2) and (7.5). Let us write out the expressions obtained there for convenience of further references:

$$
\begin{aligned}
& N_{12}|\xi\rangle=\left(\frac{1}{2}\left[a_{1}^{+}, \xi_{2}\right]\right)|\xi\rangle, \\
& N_{1}|\xi\rangle=\left(\frac{1}{2}\left[a_{1}^{+}, \xi_{1}\right]-1\right)|\xi\rangle, \\
& N_{21}|\xi\rangle=\left(\frac{1}{2}\left[a_{2}^{+}, \xi_{1}\right]\right)|\xi\rangle, \\
& N_{2}|\xi\rangle=\left(\frac{1}{2}\left[a_{2}^{+}, \xi_{2}\right]-1\right)|\xi\rangle .
\end{aligned}
$$

We note that the following relation is true:

$$
\left[a_{0}, \xi_{k}\right]=0 \text {. }
$$

Since the operator $a_{0}$ consists of only the commutators of the operators $a_{k}^{+}$and $a_{k}^{-}$, and by virtue of (B.3) and (B.4), the following relationships hold:

$\left[\left[a_{i}^{ \pm}, a_{j}^{ \pm}\right], \xi_{k}\right]=0, \quad\left[\left[a_{i}^{ \pm}, a_{j}^{\mp}\right], \xi_{k}\right]=0$.

A trivial consequence of (11.5) is the relation

$\left[\hat{A}, \xi_{k}\right]=0$,

which holds by the definition (7.1a) (or (7.1b)).

Taking into account the expressions (11.3), (11.4) and relation (11.6), we can present the matrix element of the product $\hat{A}\left[a_{0}, a_{n}^{-}\right]$as follows:

$$
\begin{aligned}
\left\langle\bar{\xi}^{\prime}\left|\hat{A}\left[a_{0}, a_{n}^{-}\right]\right| \xi\right\rangle & =\frac{1}{2}\left\{\left\langle\bar{\xi}^{\prime}\left|\hat{A}\left(\delta_{n 2} a_{1}^{+}-\delta_{n 1} a_{2}^{+}\right)\right| \xi\right\rangle\left(\frac{1}{2}\left[\xi_{1}, \xi_{2}\right]\right)\right. \\
& -\frac{1}{2} \delta_{n 1}\left[\left\langle\bar{\xi}^{\prime}\left|\hat{A} a_{2}^{+}\right| \xi\right\rangle, \xi_{1}\right] \xi_{2}-\frac{1}{2} \delta_{n 2}\left[\left\langle\bar{\xi}^{\prime}\left|\hat{A} a_{1}^{+}\right| \xi\right\rangle, \xi_{2}\right] \xi_{1} \\
& +\delta_{n 2}\left(\frac{1}{2}\left[\left\langle\bar{\xi}^{\prime}\left|\hat{A} a_{1}^{+}\right| \xi\right\rangle, \xi_{1}\right] \xi_{2}-\left\langle\bar{\xi}^{\prime}|\hat{A}| \xi\right\rangle \xi_{2}\right) \\
& +\delta_{n 1}\left(\frac{1}{2}\left[\left\langle\bar{\xi}^{\prime}\left|\hat{A} a_{2}^{+}\right| \xi\right\rangle, \xi_{2}\right] \xi_{1}-\left\langle\bar{\xi}^{\prime}|\hat{A}| \xi\right\rangle \xi_{1}\right) \\
& \left.-\left(\delta_{n 2} \xi_{2}-\delta_{n 1} \xi_{1}\right)\left\langle\bar{\xi}^{\prime}|\hat{A}| \xi\right\rangle\right\} .
\end{aligned}
$$

Thus we have been able to reduce the calculation of the initial matrix element $\left\langle\bar{\xi}^{\prime}\left|\hat{A}\left[a_{0}, a_{n}^{-}\right]\right| \xi\right\rangle$ to that of the matrix elements $\left\langle\bar{\xi}^{\prime}|\hat{A}| \xi\right\rangle$ and $\left\langle\bar{\xi}^{\prime}\left|\hat{A} a_{n}^{+}\right| \xi\right\rangle$, which in turn are given by (10.23) and (10.24), correspondingly. Collecting similar terms and recalling the definition of the derivative $\partial \Omega / \partial \bar{\xi}_{n}^{\prime}$, Eq. (10.14), we can write the expression (11.7) in a more compact form

$$
\begin{aligned}
& \left\langle\bar{\xi}^{\prime}\left|\hat{A}\left[a_{0}, a_{n}^{-}\right]\right| \xi\right\rangle \\
& \quad=\left\{-\frac{\partial \Omega}{\partial \bar{\xi}_{n}^{\prime}} \mathcal{A}+\left(\frac{\partial \Omega}{\partial \bar{\xi}_{n}^{\prime}}\right)_{\bar{\xi}_{n}^{\prime}=\partial \mathcal{A} / \partial \xi_{n}}+\xi_{n}\right\}\left\langle\bar{\xi}^{\prime} \mid \xi\right\rangle .
\end{aligned}
$$

In the second term on the right-hand side instead of variables $\bar{\xi}_{n}^{\prime}$ in the derivative (10.14) it is necessary to substitute $\partial \mathcal{A} / \partial \xi_{n}$.

Finally, we consider the operator product $\hat{A}\left[a_{0}, a_{n}^{+}\right]$. We present the matrix element of this product similar to (10.2) in the following form:

$$
\begin{aligned}
& \left\langle\bar{\xi}^{\prime}\left|\hat{A}\left[a_{0}, a_{n}^{+}\right]\right| \xi\right\rangle \\
& \quad=\left\langle\bar{\xi}^{\prime}\left|\left[a_{0}, a_{n}^{+}\right] \hat{A}\right| \xi\right\rangle+\left\langle\bar{\xi}^{\prime}\left|\left[\hat{A},\left[a_{0}, a_{n}^{+}\right]\right]\right| \xi\right\rangle .
\end{aligned}
$$

We perform analysis of the first term in the same way as it was just done for the matrix element $\left\langle\bar{\xi}^{\prime}\left|\hat{A}\left[a_{0}, a_{n}^{-}\right]\right| \xi\right\rangle$. By using the representation (11.2), we obtain

$$
\begin{aligned}
\left\langle\bar{\xi}^{\prime}\right|\left[a_{0}, a_{n}^{+}\right]= & \left\langle\bar{\xi}^{\prime}\right|\left(-\frac{1}{2}\right)\left\{\left(\frac{1}{2}\left[\bar{\xi}_{1}^{\prime}, \bar{\xi}_{2}^{\prime}\right]\right)\right. \\
& \times\left(\delta_{n 2} a_{1}^{-}-\delta_{n 1} a_{2}^{-}\right)+\left(\delta_{n 1} \bar{\xi}_{2}^{\prime} N_{12}\right. \\
& \left.+\delta_{n 2} \bar{\xi}_{1}^{\prime} N_{21}\right)-\left(\delta_{n 2} \bar{\xi}_{2}^{\prime} N_{1}+\delta_{n 1} \bar{\xi}_{1}^{\prime} N_{2}\right) \\
& \left.+\left(\delta_{n 2} \bar{\xi}_{2}^{\prime}+\delta_{n 1} \bar{\xi}_{1}^{\prime}\right)\right\} .
\end{aligned}
$$


Further, instead of (11.4), we will need the expressions

$$
\begin{aligned}
& \left\langle\bar{\xi}^{\prime}\right| N_{12}=\left\langle\bar{\xi}^{\prime}\right|\left(\frac{1}{2}\left[\bar{\xi}_{1}^{\prime}, a_{2}^{-}\right]\right), \\
& \left\langle\bar{\xi}^{\prime}\right| N_{1}=\left\langle\bar{\xi}^{\prime}\right|\left(\frac{1}{2}\left[\bar{\xi}_{1}^{\prime}, a_{1}^{-}\right]-1\right), \\
& \left\langle\bar{\xi}^{\prime}\right| N_{21}=\left\langle\bar{\xi}^{\prime}\right|\left(\frac{1}{2}\left[\bar{\xi}_{2}^{\prime}, a_{1}^{-}\right]\right), \\
& \left\langle\bar{\xi}^{\prime}\right| N_{2}=\left\langle\bar{\xi}^{\prime}\right|\left(\frac{1}{2}\left[\bar{\xi}_{2}^{\prime}, a_{2}^{-}\right]-1\right) .
\end{aligned}
$$

With these relations and by using (11.6) the first term on the right-hand side of (11.9) takes the form

$$
\begin{aligned}
\left\langle\bar{\xi}^{\prime}\right| & {\left[a_{0}, a_{n}^{+}\right] \hat{A}|\xi\rangle } \\
= & -\frac{1}{2}\left\{\left(\frac{1}{2}\left[\bar{\xi}_{1}^{\prime}, \bar{\xi}_{2}^{\prime}\right]\right)\left\langle\bar{\xi}^{\prime}\left|\left(\delta_{n 2} a_{1}^{-}-\delta_{n 1} a_{2}^{-}\right) \hat{A}\right| \xi\right\rangle\right. \\
& +\frac{1}{2} \delta_{n 1} \bar{\xi}_{2}^{\prime}\left[\bar{\xi}_{1}^{\prime},\left\langle\bar{\xi}_{1}^{\prime}\left|a_{2}^{-} \hat{A}\right| \xi\right\rangle\right] \\
& +\frac{1}{2} \delta_{n 2} \bar{\xi}_{1}^{\prime}\left[\bar{\xi}_{2}^{\prime},\left\langle\bar{\xi}_{2}^{\prime}\left|a_{1}^{-} \hat{A}\right| \xi\right\rangle\right] \\
& -\delta_{n 2}\left(\bar{\xi}_{2}^{\prime} \frac{1}{2}\left[\bar{\xi}_{1}^{\prime},\left\langle\bar{\xi}_{1}^{\prime}\left|a_{2}^{-} \hat{A}\right| \xi\right\rangle\right]-\bar{\xi}_{2}^{\prime}\left\langle\bar{\xi}^{\prime}|\hat{A}| \xi\right\rangle\right) \\
& -\delta_{n 1}\left(\bar{\xi}_{1}^{\prime} \frac{1}{2}\left[\bar{\xi}_{2}^{\prime},\left\langle\bar{\xi}_{2}^{\prime}\left|a_{1}^{-} \hat{A}\right| \xi\right\rangle\right]-\bar{\xi}_{1}^{\prime}\left\langle\bar{\xi}^{\prime}|\hat{A}| \xi\right\rangle\right) \\
& \left.+\left(\delta_{n 2} \bar{\xi}_{2}^{\prime}-\delta_{n 1} \bar{\xi}_{1}^{\prime}\right)\left\langle\bar{\xi}^{\prime}|\hat{A}| \xi\right\rangle\right\} .
\end{aligned}
$$

The last step is to use the expressions (10.23) and (10.25). Collecting similar terms and recalling the definition of the derivative $\partial \Omega / \partial \xi_{n}$, Eq. (10.11), we can write the expression above in the form similar to (11.8)

$$
\begin{aligned}
& \left\langle\bar{\xi}^{\prime}\left|\left[a_{0}, a_{n}^{+}\right] \hat{A}\right| \xi\right\rangle \\
& \quad=\left\{-\frac{\partial \Omega}{\partial \xi_{n}} \mathcal{A}+\left(\frac{\partial \Omega}{\partial \xi_{n}}\right)_{\xi_{n}=\partial \mathcal{A} / \partial \bar{\xi}_{n}^{\prime}}-\bar{\xi}_{n}^{\prime}\right\}\left\langle\bar{\xi}^{\prime} \mid \xi\right\rangle .
\end{aligned}
$$

Here, in the second term instead of the variables $\xi_{n}$ in the derivative (10.11) it is necessary to substitute $\partial \mathcal{A} / \partial \bar{\xi}_{n}^{\prime}$.

It only remains to analyse the last term in (11.9). Taking into account the second form of the operator $\hat{A}$, Eq. (7.1b), we rewrite the double commutator as follows:

$$
\begin{aligned}
{\left[\hat{A},\left[a_{0}, a_{n}^{+}\right]\right] } & =-\beta\left[a_{0},\left[a_{0}, a_{n}^{+}\right]\right]+\gamma\left[\left(a_{0}\right)^{2},\left[a_{0}, a_{n}^{+}\right]\right] \\
& =-\beta a_{n}^{+}+\gamma\left\{a_{0}, a_{n}^{+}\right\} \\
& =-\beta a_{n}^{+}+2 \gamma a_{n}^{+} a_{0}+\gamma\left[a_{0}, a_{n}^{+}\right] .
\end{aligned}
$$

Here, we have used the commutation rule (2.9). In view of (10.9) and (10.12), we get

$$
\begin{aligned}
& \left\langle\bar{\xi}^{\prime}\left|\left[\hat{A},\left[a_{0}, a_{n}^{+}\right]\right]\right| \xi\right\rangle \\
& =\left\{\bar{\xi}_{n}^{\prime}(-\beta+2 \gamma \Omega)-\gamma\left(\frac{\partial \Omega}{\partial \xi_{n}}\right)\right\}\left\langle\bar{\xi}^{\prime} \mid \xi\right\rangle .
\end{aligned}
$$

12 Connection between the matrix elements $\left\langle\bar{\xi}^{\prime}\left|a_{0}\right| \xi\right\rangle$ and $\left\langle\bar{\xi}^{\prime}\left|a_{0}^{2}\right| \xi\right\rangle$

In Sects. 7 and 5 we have derived matrix elements of the operators $a_{0}$ and $a_{0}^{2}$. For convenience of further references, we present these expressions ones again

$\left\langle\bar{\xi}^{\prime}\left|a_{0}\right| \xi\right\rangle=\Omega\left\langle\bar{\xi}^{\prime} \mid \xi\right\rangle$,

where we recall

$$
\begin{aligned}
\Omega \equiv \Omega\left(\bar{\xi}^{\prime}, \xi\right)= & -\frac{1}{2}\left\{\left(\frac{1}{2}\left[\bar{\xi}_{1}^{\prime}, \bar{\xi}_{2}^{\prime}\right]\right)\left(\frac{1}{2}\left[\xi_{1}, \xi_{2}\right]\right)\right. \\
& +\left(\frac{1}{2}\left[\bar{\xi}_{1}^{\prime}, \xi_{2}\right]\right)\left(\frac{1}{2}\left[\bar{\xi}_{2}^{\prime}, \xi_{1}\right]\right) \\
& -\left(\frac{1}{2}\left[\bar{\xi}_{1}^{\prime}, \xi_{1}\right]\right)\left(\frac{1}{2}\left[\bar{\xi}_{2}^{\prime}, \xi_{2}\right]\right) \\
& \left.+2\left(\frac{1}{2}\left[\bar{\xi}_{1}^{\prime}, \xi_{1}\right]+\frac{1}{2}\left[\bar{\xi}_{2}^{\prime}, \xi_{2}\right]-1\right)\right\}
\end{aligned}
$$

and

$\left\langle\bar{\xi}^{\prime}\left|a_{0}^{2}\right| \xi\right\rangle=\widetilde{\Omega}\left\langle\bar{\xi}^{\prime} \mid \xi\right\rangle$,

with

$$
\begin{aligned}
\widetilde{\Omega}= & \widetilde{\Omega}\left(\bar{\xi}^{\prime}, \xi\right) \\
= & 1-\sum_{k=1}^{2}\left[\left(\frac{1}{2}\left[\bar{\xi}_{k}^{\prime}, \xi_{k}\right]\right)^{2}-\frac{1}{2}\left[\bar{\xi}_{k}^{\prime}, \xi_{k}\right]+1\right] \\
& +2 \prod_{k=1}^{2}\left[\left(\frac{1}{2}\left[\bar{\xi}_{k}^{\prime}, \xi_{k}\right]\right)^{2}-\frac{1}{2}\left[\bar{\xi}_{k}^{\prime}, \xi_{k}\right]+1\right] .
\end{aligned}
$$

In this section and in the subsequent three sections we would like to establish a connection between the functions $\widetilde{\Omega}$ and $\Omega$ and thereby to clarify whether the operator $a_{0}^{2}$ is the square of the operator $a_{0}$. For this purpose, we use the insertion of resolution of the identity operator

$\iint|\mu\rangle\langle\bar{\mu}| \mathrm{e}^{-\frac{1}{2}[\bar{\mu}, \mu]}(d \mu)_{2}(d \bar{\mu})_{2}=\hat{1}$,

where $\bar{\mu}, \mu$ are para-Grassmann numbers,

$(d \mu)_{2} \equiv d^{2} \mu_{2} d^{2} \mu_{1}, \quad(d \bar{\mu})_{2} \equiv d^{2} \bar{\mu}_{1} d^{2} \bar{\mu}_{2}$

are the measure of integration and as usual, for the sake of brevity we make use of the notations

$[\bar{\mu}, \mu]=\sum_{k=1}^{2}\left[\bar{\mu}_{k}, \mu_{k}\right]$

and so on. Then, by virtue of the definition (12.1) we get

$$
\begin{aligned}
\left\langle\bar{\xi}^{\prime}\left|a_{0}^{2}\right| \xi\right\rangle= & \iint\left\langle\bar{\xi}^{\prime}\left|a_{0}\right| \mu\right\rangle\left\langle\bar{\mu}\left|a_{0}\right| \xi\right\rangle \\
& \times \mathrm{e}^{-\frac{1}{2}[\bar{\mu}, \mu]}(d \mu)_{2}(d \bar{\mu})_{2} \\
= & \iint \Omega\left(\bar{\xi}^{\prime}, \mu\right) \Omega(\bar{\mu}, \xi) \mathrm{e}^{-\frac{1}{2}\left[\bar{\mu}-\bar{\xi}^{\prime}, \mu-\xi\right]}
\end{aligned}
$$




$$
\times(d \mu)_{2}(d \bar{\mu})_{2} \mathrm{e}^{\frac{1}{2}\left[\bar{\xi}^{\prime}, \xi\right]} .
$$

Here, we have written down an explicit form of the overlap function (4.8). Substituting the representation (12.3) into the left-hand side of this expression and canceling the factor $\left\langle\bar{\xi}^{\prime} \mid \xi\right\rangle$, we obtain

$$
\begin{aligned}
\widetilde{\Omega}\left(\bar{\xi}^{\prime}, \xi\right)= & \iint \Omega\left(\bar{\xi}^{\prime}, \mu\right) \Omega(\bar{\mu}, \xi) \\
& \times \mathrm{e}^{-\frac{1}{2}\left[\bar{\mu}-\bar{\xi}^{\prime}, \mu-\xi\right]}(d \mu)_{2}(d \bar{\mu})_{2} .
\end{aligned}
$$

The last step is a shift of the variables of integration:

$\bar{\mu} \rightarrow \bar{\mu}+\bar{\xi}^{\prime}, \quad \mu \rightarrow \mu+\xi$

and finally we get

$$
\begin{aligned}
\widetilde{\Omega}\left(\bar{\xi}^{\prime}, \xi\right)= & \iint \Omega\left(\bar{\xi}^{\prime}, \xi+\mu\right) \Omega\left(\bar{\xi}^{\prime}+\bar{\mu}, \xi\right) \\
& \times \mathrm{e}^{-\frac{1}{2}[\bar{\mu}, \mu]}(d \mu)_{2}(d \bar{\mu})_{2} .
\end{aligned}
$$

By these means, it is necessary to take the integral on the right-hand side of (12.5) for the function $\Omega$ defined by the expression (12.2) and to compare the obtained expression with (12.4).

The first step is an expansion of the function $\Omega$ in the integrand (12.5) in powers of $\mu$ and $\bar{\mu}$. From (12.2) it is easy to see that these expansions have the following form:

$$
\begin{aligned}
& \Omega\left(\bar{\xi}^{\prime}, \xi+\mu\right) \\
&=\Omega\left(\bar{\xi}^{\prime}, \xi\right)-\frac{1}{2}\left[\frac{\partial \Omega\left(\bar{\xi}^{\prime}, \xi\right)}{\partial \xi}, \mu\right]+\left(-\frac{1}{2^{3}}\right) \\
& \times\left\{\left[\bar{\xi}_{1}^{\prime}, \bar{\xi}_{2}^{\prime}\right]\left[\mu_{1}, \mu_{2}\right]+\left[\bar{\xi}_{2}^{\prime}, \mu_{1}\right]\left[\bar{\xi}_{1}^{\prime}, \mu_{2}\right]\right. \\
&\left.-\left[\bar{\xi}_{1}^{\prime}, \mu_{1}\right]\left[\bar{\xi}_{2}^{\prime}, \mu_{2}\right]\right\}
\end{aligned}
$$

and

$$
\begin{aligned}
\Omega & \left(\bar{\xi}^{\prime}+\bar{\mu}, \xi\right) \\
= & \Omega\left(\bar{\xi}^{\prime}, \xi\right)+\frac{1}{2}\left[\bar{\mu}, \frac{\partial \Omega\left(\bar{\xi}^{\prime}, \xi\right)}{\partial \bar{\xi}^{\prime}}\right]+\left(-\frac{1}{2^{3}}\right) \\
& \times\left\{\left[\bar{\mu}_{1}, \bar{\mu}_{2}\right]\left[\xi_{1}, \xi_{2}\right]+\left[\bar{\mu}_{2}, \xi_{1}\right]\left[\bar{\mu}_{1}, \xi_{2}\right]\right. \\
& \left.-\left[\bar{\mu}_{1}, \xi_{1}\right]\left[\bar{\mu}_{2}, \xi_{2}\right]\right\} .
\end{aligned}
$$

We have introduced the markers (1), (2) and (3) over the terms which are quadratic in $\mu$ and $\bar{\mu}$.
Let us consider the terms in (12.6) and (12.7) linear in $\mu$ and $\bar{\mu}$. Substituting them into (12.5), one obtains

$$
\begin{aligned}
& \Omega\left(\bar{\xi}^{\prime}, \xi\right) \iint\left(\left[\bar{\mu}, \frac{\partial \Omega\left(\bar{\xi}^{\prime}, \xi\right)}{\partial \bar{\xi}^{\prime}}\right]-\left[\frac{\partial \Omega\left(\bar{\xi}^{\prime}, \xi\right)}{\partial \xi}, \mu\right]\right) \\
& \quad \times \mathrm{e}^{-\frac{1}{2}[\bar{\mu}, \mu]}(d \mu)_{2}(d \bar{\mu})_{2} \\
& =\Omega\left(\bar{\xi}^{\prime}, \xi\right)\left(\int\left[\bar{\mu}, \frac{\partial \Omega\left(\bar{\xi}^{\prime}, \xi\right)}{\partial \bar{\xi}^{\prime}}\right] \delta^{(2)}(\bar{\mu})(d \bar{\mu})_{2}\right. \\
& \left.\quad-\int\left[\frac{\partial \Omega\left(\bar{\xi}^{\prime}, \xi\right)}{\partial \xi}, \mu\right] \delta^{(2)}(\mu)(d \mu)_{2}\right)=0 .
\end{aligned}
$$

Here, the para-Grassmann $\delta$-functions are

$\delta^{(2)}(\bar{\mu}) \equiv \delta\left(\bar{\mu}_{1}\right) \delta\left(\bar{\mu}_{2}\right), \quad \delta^{(2)}(\mu) \equiv \delta\left(\mu_{1}\right) \delta\left(\mu_{2}\right)$,

where $\delta\left(\bar{\mu}_{i}\right)$ and $\delta\left(\mu_{i}\right), i=1,2$, are defined by the formula (D.5).

Further we consider the contributions quadratic in $\mu$ and $\bar{\mu}$. At first we calculate a contribution with derivatives of the function $\Omega$ :

$$
\begin{aligned}
& -\frac{1}{2^{2}} \iint\left[\frac{\partial \Omega\left(\bar{\xi}^{\prime}, \xi\right)}{\partial \xi}, \mu\right]\left[\bar{\mu}, \frac{\partial \Omega\left(\bar{\xi}^{\prime}, \xi\right)}{\partial \bar{\xi}^{\prime}}\right] \\
& \times \mathrm{e}^{-\frac{1}{2}[\bar{\mu}, \mu]}(d \mu)_{2}(d \bar{\mu})_{2} .
\end{aligned}
$$

There are two independent ways of taking the integrals of this type. The first of them was suggested in the paper by Omote and Kamefuchi [25]. We take the integral (12.8) by the first way and then verify the result of calculations by another way.

Following an approach suggested by Omote and Kamefuchi, we present the exponential in the integrand (12.8) in the form of the overlap function

$\mathrm{e}^{-\frac{1}{2}[\bar{\mu}, \mu]}=\langle-\bar{\mu} \mid \mu\rangle$.

Then, instead of (12.8), we will have a chain of equalities

$$
\begin{aligned}
- & \frac{1}{2^{2}} \iint\left(\left[\frac{\partial \Omega\left(\bar{\xi}^{\prime}, \xi\right)}{\partial \xi_{1}}, \mu_{1}\right]+\left[\frac{\partial \Omega\left(\bar{\xi}^{\prime}, \xi\right)}{\partial \xi_{2}}, \mu_{2}\right]\right) \\
& \times\langle-\bar{\mu} \mid \mu\rangle(d \mu)_{2}\left[\bar{\mu}, \frac{\partial \Omega\left(\bar{\xi}^{\prime}, \xi\right)}{\partial \bar{\xi}^{\prime}}\right](d \bar{\mu})_{2} \\
= & -\frac{1}{2^{2}} \iint\langle-\bar{\mu}|\left(\left[\frac{\partial \Omega\left(\bar{\xi}^{\prime}, \xi\right)}{\partial \xi_{1}}, a_{1}^{-}\right]\right. \\
& \left.+\left[\frac{\partial \Omega\left(\bar{\xi}^{\prime}, \xi\right)}{\partial \xi_{2}}, a_{2}^{-}\right]\right)|\mu\rangle(d \mu)_{2}\left[\bar{\mu}, \frac{\partial \Omega\left(\bar{\xi}^{\prime}, \xi\right)}{\partial \bar{\xi}^{\prime}}\right](d \bar{\mu})_{2} \\
= & -\frac{1}{2^{4}} \iint\langle-\bar{\mu}|\left(\left[\frac{\partial \Omega\left(\bar{\xi}^{\prime}, \xi\right)}{\partial \xi_{1}}, a_{1}^{-}\right]+\left[\frac{\partial \Omega\left(\bar{\xi}^{\prime}, \xi\right)}{\partial \xi_{2}}, a_{2}^{-}\right]\right) \\
& \times\left(a_{1}^{+}\right)^{2}\left(a_{2}^{+}\right)^{2}|0\rangle(d \mu)_{2}\left[\bar{\mu}, \frac{\partial \Omega\left(\bar{\xi}^{\prime}, \xi\right)}{\partial \bar{\xi}^{\prime}}\right](d \bar{\mu})_{2} .
\end{aligned}
$$

Here, at the last step we have formally used the definition of $\delta$-function (D.5) as applied to the creation operator $a_{k}^{+}$:

$$
\begin{aligned}
\int|\mu\rangle(d \mu)_{2} & =\int \mathrm{e}^{-\frac{1}{2}\left[\mu, a^{+}\right]}|0\rangle(d \mu)_{2} \\
& =\frac{1}{2^{2}}\left(a_{1}^{+}\right)^{2}\left(a_{2}^{+}\right)^{2}|0\rangle .
\end{aligned}
$$


The last matrix element in (12.10) is calculated when the operators $a_{1}^{-}$and $a_{2}^{-}$are shifted, with the use of (B.3) and (B.4), to the right until the vacuum conditions can be employed. The result of calculations is

$$
\begin{aligned}
& \left\langle-\bar{\mu}\left|\left(\left[\frac{\partial \Omega\left(\bar{\xi}^{\prime}, \xi\right)}{\partial \xi_{1}}, a_{1}^{-}\right]+\left[\frac{\partial \Omega\left(\bar{\xi}^{\prime}, \xi\right)}{\partial \xi_{2}}, a_{2}^{-}\right]\right)\left(a_{1}^{+}\right)^{2}\left(a_{2}^{+}\right)^{2}\right| 0\right\rangle \\
& =2^{2}\left(\delta\left(\bar{\mu}_{2}\right)\left\{\frac{\partial \Omega\left(\bar{\xi}^{\prime}, \xi\right)}{\partial \xi_{1}}, \bar{\mu}_{1}\right\}+\delta\left(\bar{\mu}_{1}\right)\left\{\frac{\partial \Omega\left(\bar{\xi}^{\prime}, \xi\right)}{\partial \xi_{2}}, \bar{\mu}_{2}\right\}\right) .
\end{aligned}
$$

The remaining integral with respect to $(d \bar{\mu})_{2}$ in (12.10) is easily calculated by using the formula (D.4)

$$
\begin{aligned}
- & \frac{1}{2^{2}} \int\left[\bar{\mu}, \frac{\partial \Omega\left(\bar{\xi}^{\prime}, \xi\right)}{\partial \bar{\xi}^{\prime}}\right]\left(\delta\left(\bar{\mu}_{2}\right)\left\{\frac{\partial \Omega\left(\bar{\xi}^{\prime}, \xi\right)}{\partial \xi_{1}}, \bar{\mu}_{1}\right\}\right. \\
& \left.+\delta\left(\bar{\mu}_{1}\right)\left\{\frac{\partial \Omega\left(\bar{\xi}^{\prime}, \xi\right)}{\partial \xi_{2}}, \bar{\mu}_{2}\right\}\right) d^{2} \bar{\mu}_{1} d^{2} \bar{\mu}_{2} \\
= & -\frac{1}{2^{2}}\left(\int\left[\bar{\mu}_{1}, \frac{\partial \Omega\left(\bar{\xi}^{\prime}, \xi\right)}{\partial \bar{\xi}_{1}^{\prime}}\right]\left\{\frac{\partial \Omega\left(\bar{\xi}^{\prime}, \xi\right)}{\partial \xi_{1}}, \bar{\mu}_{1}\right\} d^{2} \bar{\mu}_{1}\right. \\
& \left.+\int\left[\bar{\mu}_{2}, \frac{\partial \Omega\left(\bar{\xi}^{\prime}, \xi\right)}{\partial \bar{\xi}_{2}^{\prime}}\right]\left\{\frac{\partial \Omega\left(\bar{\xi}^{\prime}, \xi\right)}{\partial \xi_{2}}, \bar{\mu}_{2}\right\} d^{2} \bar{\mu}_{2}\right) \\
= & -\frac{1}{2}\left(\left[\frac{\partial \Omega\left(\bar{\xi}^{\prime}, \xi\right)}{\partial \xi_{1}}, \frac{\partial \Omega\left(\bar{\xi}^{\prime}, \xi\right)}{\partial \bar{\xi}_{1}^{\prime}}\right]\right. \\
& \left.+\left[\frac{\partial \Omega\left(\bar{\xi}^{\prime}, \xi\right)}{\partial \xi_{2}}, \frac{\partial \Omega\left(\bar{\xi}^{\prime}, \xi\right)}{\partial \bar{\xi}_{2}^{\prime}}\right]\right) \\
\equiv & -\frac{1}{2}\left[\frac{\partial \Omega\left(\bar{\xi}^{\prime}, \xi\right)}{\partial \xi}, \frac{\partial \Omega\left(\bar{\xi}^{\prime}, \xi\right)}{\partial \bar{\xi}^{\prime}}\right] .
\end{aligned}
$$

Therefore, we can already write out the first two terms in the expansion of convolution integral (12.5)

$$
\begin{aligned}
\widetilde{\Omega}\left(\bar{\xi}^{\prime}, \xi\right)= & {\left[\Omega\left(\bar{\xi}^{\prime}, \xi\right)\right]^{2}+\left(-\frac{1}{2}\right) } \\
& \times\left[\frac{\partial \Omega\left(\bar{\xi}^{\prime}, \xi\right)}{\partial \xi}, \frac{\partial \Omega\left(\bar{\xi}^{\prime}, \xi\right)}{\partial \bar{\xi}^{\prime}}\right]+\cdots .
\end{aligned}
$$

Let us analyse the contributions cubic in $\mu$ and $\bar{\mu}$. We take for example the term with derivative from the expansion (12.6) and the contribution (1) from (12.7). Then, instead of (12.8), we have

$$
\begin{aligned}
& \frac{1}{2^{4}}\left[\xi_{1}, \xi_{2}\right] \iint\left[\frac{\partial \Omega\left(\bar{\xi}^{\prime}, \xi\right)}{\partial \xi}, \mu\right]\left[\bar{\mu}_{1}, \bar{\mu}_{2}\right] \\
& \quad \times \mathrm{e}^{-\frac{1}{2}[\bar{\mu}, \mu]}(d \mu)_{2}(d \bar{\mu})_{2} \\
& =\frac{1}{2^{4}}\left[\xi_{1}, \xi_{2}\right] \iint\left(\delta\left(\bar{\mu}_{2}\right)\left\{\frac{\partial \Omega\left(\bar{\xi}^{\prime}, \xi\right)}{\partial \xi_{1}}, \bar{\mu}_{1}\right\}\right. \\
& \left.\quad+\delta\left(\bar{\mu}_{1}\right)\left\{\frac{\partial \Omega\left(\bar{\xi}^{\prime}, \xi\right)}{\partial \xi_{2}}, \bar{\mu}_{2}\right\}\right) \\
& \quad \times\left[\bar{\mu}_{1}, \bar{\mu}_{2}\right] d^{2} \bar{\mu}_{1} d^{2} \bar{\mu}_{2}=0 .
\end{aligned}
$$

Here, we have used the results of the previous calculations, Eqs. (12.10) and (12.11). Similarly, we can verify that in
(12.5) the remaining contributions cubic in $\mu$ and $\bar{\mu}$ also vanish.

\section{Contributions of the fourth order in $\mu$ and $\bar{\mu}$}

Now we turn to the consideration of the remaining contributions of fourth order in $\mu$ and $\bar{\mu}$. Let us consider the "diagonal" contribution (1) - (1), where the first one (1) designates a term with a mark (1) in (12.6) and the second one (1) denotes a similar term in (12.7):

$$
\begin{aligned}
& \frac{1}{2^{6}}\left[\bar{\xi}_{1}^{\prime}, \bar{\xi}_{2}^{\prime}\right]\left[\xi_{1}, \xi_{2}\right] \iint\left[\mu_{1}, \mu_{2}\right]\left[\bar{\mu}_{1}, \bar{\mu}_{2}\right] \\
& \quad \times \mathrm{e}^{-\frac{1}{2}[\bar{\mu}, \mu]}(d \mu)_{2}(d \bar{\mu})_{2} .
\end{aligned}
$$

The use of the representation (12.9) and the expression like (12.10) leads to necessity of the calculation of the matrix element

$\left\langle-\bar{\mu}\left|\left[a_{1}^{-}, a_{2}^{-}\right]\left(a_{2}^{+}\right)^{2}\left(a_{1}^{+}\right)^{2}\right| 0\right\rangle$.

In view of the commutation rules (2.4)-(2.6), we obtain

$$
\begin{aligned}
& {\left[a_{1}^{-}, a_{2}^{-}\right]\left(a_{2}^{+}\right)^{2}\left(a_{1}^{+}\right)^{2}} \\
& \quad=\left(a_{2}^{+}\right)^{2}\left(a_{1}^{+}\right)^{2}\left[a_{1}^{-}, a_{2}^{-}\right]-2\left(a_{2}^{+}\right)^{2} a_{2}^{-} a_{1}^{+}-2 a_{1}^{+} a_{2}^{+} a_{1}^{-} a_{1}^{+} \\
& \quad-2 a_{2}^{+} a_{1}^{+} a_{1}^{+} a_{1}^{-}+4 a_{2}^{+} a_{1}^{+}-2\left(a_{2}^{+}\right)^{2} a_{1}^{+} a_{2}^{-},
\end{aligned}
$$

and therefore the required matrix element equals

$$
\begin{aligned}
& \left\langle-\bar{\mu}\left|\left[a_{1}^{-}, a_{2}^{-}\right]\left(a_{2}^{+}\right)^{2}\left(a_{1}^{+}\right)^{2}\right| 0\right\rangle \\
& \quad=-4\left[\bar{\mu}_{1}, \bar{\mu}_{2}\right],
\end{aligned}
$$

and the integral (13.1) transforms to

$$
\begin{aligned}
& -\frac{1}{2^{6}}\left[\bar{\xi}_{1}^{\prime}, \bar{\xi}_{2}^{\prime}\right]\left[\xi_{1}, \xi_{2}\right] \iint\left[\bar{\mu}_{1}, \bar{\mu}_{2}\right]^{2} d^{2} \bar{\mu}_{1} d^{2} \bar{\mu}_{2} \\
& =-\frac{1}{2^{3}}\left[\bar{\xi}_{1}^{\prime}, \bar{\xi}_{2}^{\prime}\right]\left[\xi_{1}, \xi_{2}\right] \int \delta\left(\bar{\mu}_{2}\right) d^{2} \bar{\mu}_{2} .
\end{aligned}
$$

Here, we have used the integration formula (D.3) and the definition of $\delta$-function, Eq.(D.5). As a result, the desired contribution of fourth order (1) - (1) is equal to

(1)-(1): $-\frac{1}{2^{3}}\left[\bar{\xi}_{1}^{\prime}, \bar{\xi}_{2}^{\prime}\right]\left[\xi_{1}, \xi_{2}\right]$.

Let us consider the second "diagonal" contribution of fourth order to the convolution (12.5), namely (2)-(2) one:

$$
\begin{aligned}
& \frac{1}{2^{6}} \iint\left[\bar{\xi}_{2}^{\prime}, \mu_{1}\right]\left[\bar{\xi}_{1}^{\prime}, \mu_{2}\right]\left[\bar{\mu}_{2}, \xi_{1}\right]\left[\bar{\mu}_{1}, \xi_{2}\right] \\
& \mathrm{e}^{-\frac{1}{2}[\bar{\mu}, \mu]}(d \mu)_{2}(d \bar{\mu})_{2} .
\end{aligned}
$$

Here, we are faced with an analysis of matrix element of the following type:

$$
\left\langle-\bar{\mu}\left|\left[\bar{\xi}_{2}^{\prime}, a_{1}^{-}\right]\left[\bar{\xi}_{1}^{\prime}, a_{2}^{-}\right]\left(a_{2}^{+}\right)^{2}\left(a_{1}^{+}\right)^{2}\right| 0\right\rangle .
$$


By using the commutation rules (B.3) and (B.4), after somewhat cumbersome calculations we find

$$
\begin{aligned}
{\left[\bar{\xi}_{2}^{\prime}, a_{1}^{-}\right]\left[\bar{\xi}_{1}^{\prime}, a_{2}^{-}\right]\left(a_{2}^{+}\right)^{2}\left(a_{1}^{+}\right)^{2} } \\
=\left(a_{2}^{+}\right)^{2}\left(a_{1}^{+}\right)^{2}\left[\bar{\xi}_{2}^{\prime}, a_{1}^{-}\right]\left[\bar{\xi}_{1}^{\prime}, a_{2}^{-}\right] \\
\quad+2\left(\bar{\xi}_{2}^{\prime} a_{1}^{+}\left(a_{2}^{+}\right)^{2}\left[\bar{\xi}_{1}^{\prime}, a_{2}^{-}\right]+a_{1}^{+} \bar{\xi}_{2}^{\prime}\left(a_{2}^{+}\right)^{2}\left[\bar{\xi}_{1}^{\prime}, a_{2}^{-}\right]\right. \\
\left.\quad+\left(a_{1}^{+}\right)^{2} \bar{\xi}_{1}^{\prime} a_{2}^{+}\left[\bar{\xi}_{2}^{\prime}, a_{1}^{-}\right]+\left(a_{1}^{+}\right)^{2} a_{2}^{+} \bar{\xi}_{1}^{\prime}\left[\bar{\xi}_{2}^{\prime}, a_{1}^{-}\right]\right) \\
\quad+4\left(\bar{\xi}_{2}^{\prime} a_{1}^{+} \bar{\xi}_{1}^{\prime} a_{2}^{+}+a_{1}^{+} \bar{\xi}_{2}^{\prime} \bar{\xi}_{1}^{\prime} a_{2}^{+}+\bar{\xi}_{2}^{\prime} a_{1}^{+} a_{2}^{+} \bar{\xi}_{1}^{\prime}+a_{1}^{+} \bar{\xi}_{2}^{\prime} a_{2}^{+} \bar{\xi}_{1}^{\prime}\right) .
\end{aligned}
$$

Substituting this expression into (13.4), we obtain

$$
\begin{aligned}
& \left\langle-\bar{\mu}\left|\left[\bar{\xi}_{2}^{\prime}, a_{1}^{-}\right]\left[\bar{\xi}_{1}^{\prime}, a_{2}^{-}\right]\left(a_{2}^{+}\right)^{2}\left(a_{1}^{+}\right)^{2}\right| 0\right\rangle \\
& \quad=4\left(\bar{\xi}_{2}^{\prime} \bar{\mu}_{1} \bar{\xi}_{1}^{\prime} \bar{\mu}_{2}+\bar{\mu}_{1} \bar{\xi}_{2}^{\prime} \bar{\xi}_{1}^{\prime} \bar{\mu}_{2}+\bar{\xi}_{2}^{\prime} \bar{\mu}_{1} \bar{\mu}_{2} \bar{\xi}_{1}^{\prime}+\bar{\mu}_{1} \bar{\xi}_{2}^{\prime} \bar{\mu}_{2} \bar{\xi}_{1}^{\prime}\right) \\
& \quad \equiv 4\left\{\bar{\mu}_{1}, \bar{\xi}_{2}^{\prime}\right\}\left\{\bar{\mu}_{2}, \bar{\xi}_{1}^{\prime}\right\} .
\end{aligned}
$$

In this case, the integral (13.3) is reduced to a product of two independent integrals:

$$
\begin{aligned}
& \frac{1}{2^{6}}\left(\int\left[\bar{\mu}_{1}, \xi_{2}\right]\left\{\bar{\mu}_{1}, \bar{\xi}_{2}^{\prime}\right\} d^{2} \bar{\mu}_{1}\right)\left(\int\left[\bar{\mu}_{2}, \xi_{1}\right]\left\{\bar{\mu}_{2}, \bar{\xi}_{1}^{\prime}\right\} d^{2} \bar{\mu}_{2}\right) \\
& \quad=\frac{1}{2^{4}}\left[\bar{\xi}_{1}^{\prime}, \xi_{1}\right]\left[\bar{\xi}_{2}^{\prime}, \xi_{2}\right] .
\end{aligned}
$$

Here, we have used the integration formula (D.4). As a result, for the contribution (2) - (2) we derive

$(2)-(2): \frac{1}{2^{4}}\left[\bar{\xi}_{1}^{\prime}, \xi_{1}\right]\left[\bar{\xi}_{2}^{\prime}, \xi_{2}\right]$.

From the expressions (12.6) and (12.7) it is easy to see that the third "diagonal" contribution $(3)-(3)$ follows from previous one by a simple replacement: $\bar{\xi}_{1}^{\prime} \rightleftarrows \bar{\xi}_{2}^{\prime}, \xi_{1} \rightleftarrows \xi_{2}$, consequently we can immediately write

(3)-(3): $\frac{1}{2^{4}}\left[\bar{\xi}_{1}^{\prime}, \xi_{1}\right]\left[\bar{\xi}_{2}^{\prime}, \xi_{2}\right]$.

Let us now examine the fourth order "off-diagonal" contributions. The calculations completely similar to the previous ones result in the following expressions:

$$
\begin{aligned}
& (\mathbf{1})-(\mathbf{2})=(\mathbf{2})-(\mathbf{1}): \quad-\frac{1}{2^{4}}\left[\bar{\xi}_{1}^{\prime}, \bar{\xi}_{2}^{\prime}\right]\left[\xi_{1}, \xi_{2}\right], \\
& (\mathbf{1})-(\mathbf{3})=(\mathbf{3})-(\mathbf{1}): \quad-\frac{1}{2^{4}}\left[\bar{\xi}_{1}^{\prime}, \bar{\xi}_{2}^{\prime}\right]\left[\xi_{1}, \xi_{2}\right], \\
& (\mathbf{2})-(\mathbf{3})=(\mathbf{3})-(\mathbf{2}): \quad-\frac{1}{2^{4}}\left[\bar{\xi}_{1}^{\prime}, \xi_{2}\right]\left[\bar{\xi}_{2}^{\prime}, \xi_{1}\right] .
\end{aligned}
$$

Substituting the obtained terms of expansion (12.13), (13.2), (13.5), (13.6) and (13.7) into (12.5) and collecting similar terms, we finally obtain

$$
\begin{aligned}
\widetilde{\Omega}\left(\bar{\xi}^{\prime}, \xi\right)= & {\left[\Omega\left(\bar{\xi}^{\prime}, \xi\right)\right]^{2} } \\
& +\left(-\frac{1}{2}\right)\left[\frac{\partial \Omega\left(\bar{\xi}^{\prime}, \xi\right)}{\partial \xi}, \frac{\partial \Omega\left(\bar{\xi}^{\prime}, \xi\right)}{\partial \bar{\xi}^{\prime}}\right] \\
& +\left(-\frac{3}{2^{3}}\right)\left[\bar{\xi}_{1}^{\prime}, \bar{\xi}_{2}^{\prime}\right]\left[\xi_{1}, \xi_{2}\right] \\
& +\frac{1}{2^{3}}\left[\bar{\xi}_{1}^{\prime}, \xi_{1}\right]\left[\bar{\xi}_{2}^{\prime}, \xi_{2}\right]
\end{aligned}
$$

$$
+\left(-\frac{1}{2^{3}}\right)\left[\bar{\xi}_{1}^{\prime}, \xi_{2}\right]\left[\bar{\xi}_{2}^{\prime}, \xi_{1}\right]
$$

This expression can be rewritten in terms of derivatives of the function $\Omega$. Making use of the differentiation formulas (C.1)-(C.4) and an explicit form of the function (12.2), we find instead of (13.8)

$$
\begin{aligned}
\widetilde{\Omega}\left(\bar{\xi}^{\prime}, \xi\right)= & {\left[\Omega\left(\bar{\xi}^{\prime}, \xi\right)\right]^{2} } \\
& +\left(-\frac{1}{2}\right)\left[\frac{\partial \Omega\left(\bar{\xi}^{\prime}, \xi\right)}{\partial \xi}, \frac{\partial \Omega\left(\bar{\xi}^{\prime}, \xi\right)}{\partial \bar{\xi}^{\prime}}\right] \\
& +\left(-\frac{3}{2^{3}}\right)\left(\frac{\partial^{2} \Omega\left(\bar{\xi}^{\prime}, \xi\right)}{\partial \xi_{1} \partial \xi_{2}} \cdot \frac{\partial^{2} \Omega\left(\bar{\xi}^{\prime}, \xi\right)}{\partial \bar{\xi}_{1}^{\prime} \partial \bar{\xi}_{2}^{\prime}}\right) \\
& +\frac{1}{2^{3}}\left(\frac{\partial^{2} \Omega\left(\bar{\xi}^{\prime}, \xi\right)}{\partial \bar{\xi}_{1}^{\prime} \partial \xi_{1}}-2\right)\left(\frac{\partial^{2} \Omega\left(\bar{\xi}^{\prime}, \xi\right)}{\partial \bar{\xi}_{2}^{\prime} \partial \xi_{2}}-2\right) \\
& +\left(-\frac{1}{2^{3}}\right)\left(\frac{\partial^{2} \Omega\left(\bar{\xi}^{\prime}, \xi\right)}{\partial \bar{\xi}_{1}^{\prime} \partial \xi_{2}} \cdot \frac{\partial^{2} \Omega\left(\bar{\xi}^{\prime}, \xi\right)}{\partial \bar{\xi}_{2}^{\prime} \partial \xi_{1}}\right) .
\end{aligned}
$$

In closing this section, we would like to consider another more direct way of taking the integrals with the paraGrassmann variables. As an example, let us examine the contribution (1)-(1), i.e. the expression (13.1). Instead of representation (12.9) we use the expansion of the exponential function

$$
\begin{aligned}
\mathrm{e}^{-\frac{1}{2}[\bar{\mu}, \mu]}= & 1-\frac{1}{2}[\bar{\mu}, \mu]+\frac{1}{2 !}\left(\frac{1}{2}\right)^{2}[\bar{\mu}, \mu]^{2} \\
& -\frac{1}{3 !}\left(\frac{1}{2}\right)^{3}[\bar{\mu}, \mu]^{3}+\frac{1}{4 !}\left(\frac{1}{2}\right)^{4}[\bar{\mu}, \mu]^{4} \\
= & 1-\frac{1}{2}\left(\left[\bar{\mu}_{1}, \mu_{1}\right]+\left[\bar{\mu}_{2}, \mu_{2}\right]\right) \\
& +\frac{1}{2 !}\left(\frac{1}{2}\right)^{2}\left(\left[\bar{\mu}_{1}, \mu_{1}\right]^{2}\right. \\
& \left.+2\left[\bar{\mu}_{1}, \mu_{1}\right]\left[\bar{\mu}_{2}, \mu_{2}\right]+\left[\bar{\mu}_{2}, \mu_{2}\right]^{2}\right) \\
& -\frac{1}{3 !}\left(\frac{1}{2}\right)^{3}\left(3\left[\bar{\mu}_{1}, \mu_{1}\right]^{2}\left[\bar{\mu}_{2}, \mu_{2}\right]\right. \\
& \left.+3\left[\bar{\mu}_{1}, \mu_{1}\right]\left[\bar{\mu}_{2}, \mu_{2}\right]^{2}\right) \\
& +\frac{1}{4 !}\left(\frac{1}{2}\right)^{4} 6\left[\bar{\mu}_{1}, \mu_{1}\right]^{2}\left[\bar{\mu}_{2}, \mu_{2}\right]^{2}
\end{aligned}
$$

As it is not difficult to see from the integration formulae (D.1)-(D.4), a nontrivial contribution to the expression (13.1) for $p=2$ gives us only one term from the right-hand side (13.9), namely

$\frac{1}{2^{2}}\left[\bar{\mu}_{1}, \mu_{1}\right]\left[\bar{\mu}_{2}, \mu_{2}\right]$,

and therefore by using subsequently (D.3), (D.4) and (D.5), we derive

$$
\iint\left[\mu_{1}, \mu_{2}\right]\left[\bar{\mu}_{1}, \bar{\mu}_{2}\right] \mathrm{e}^{-\frac{1}{2}[\bar{\mu}, \mu]}(d \mu)_{2}(d \bar{\mu})_{2}
$$




$$
\begin{aligned}
& =\frac{1}{2^{2}} \iint\left[\mu_{1}, \mu_{2}\right]\left[\bar{\mu}_{1}, \bar{\mu}_{2}\right]\left[\bar{\mu}_{1}, \mu_{1}\right]\left[\bar{\mu}_{2}, \mu_{2}\right](d \mu)_{2}(d \bar{\mu})_{2} \\
& =\frac{1}{2^{2}} 2 i^{2} \iint\left[\bar{\mu}_{1}, \bar{\mu}_{2}\right]\left[\bar{\mu}_{1}, \mu_{1}\right]\left\{\mu_{1}, \bar{\mu}_{2}\right\} d^{2} \mu_{1}(d \bar{\mu})_{2} \\
& =\frac{1}{2^{2}}\left(2 i^{2}\right)^{2} \iint\left[\bar{\mu}_{1}, \bar{\mu}_{2}\right]\left[\bar{\mu}_{2}, \bar{\mu}_{1}\right] d^{2} \bar{\mu}_{1} d^{2} \bar{\mu}_{2} \\
& =-\frac{1}{2^{2}} 2^{5} \int \delta\left(\bar{\mu}_{2}\right) d^{2} \bar{\mu}_{2}=-2^{3} .
\end{aligned}
$$

Substituting the obtained number into (13.1), we reproduce (13.2).

Let us verify more nontrivial contribution (12.8). At the beginning we write in more detail the integrand in (12.8):

$$
\begin{aligned}
{\left[\frac{\partial \Omega}{\partial \xi}, \mu\right]\left[\bar{\mu}, \frac{\partial \Omega}{\partial \bar{\xi}^{\prime}}\right]=} & {\left[\frac{\partial \Omega}{\partial \xi_{1}}, \mu_{1}\right]\left[\bar{\mu}_{1}, \frac{\partial \Omega}{\partial \bar{\xi}_{1}^{\prime}}\right] } \\
& +\left[\frac{\partial \Omega}{\partial \xi_{2}}, \mu_{2}\right]\left[\bar{\mu}_{2}, \frac{\partial \Omega}{\partial \bar{\xi}_{2}^{\prime}}\right] \\
& +\left[\frac{\partial \Omega}{\partial \xi_{1}}, \mu_{1}\right]\left[\bar{\mu}_{2}, \frac{\partial \Omega}{\partial \bar{\xi}_{2}^{\prime}}\right] \\
& +\left[\frac{\partial \Omega}{\partial \xi_{2}}, \mu_{2}\right]\left[\bar{\mu}_{1}, \frac{\partial \Omega}{\partial \bar{\xi}_{1}^{\prime}}\right] .
\end{aligned}
$$

For the first two terms on the right-hand side of (13.10) in the expansion (13.9) only two terms give a nontrivial contribution:

$-\frac{1}{2^{4}}\left(\left[\bar{\mu}_{1}, \mu_{1}\right]^{2}\left[\bar{\mu}_{2}, \mu_{2}\right]+\left[\bar{\mu}_{1}, \mu_{1}\right]\left[\bar{\mu}_{2}, \mu_{2}\right]^{2}\right)$.

For the remaining terms in (13.10) there are no such nontrivial contributions in (13.9) and therefore they can be dropped. The integral with the first term on the right-hand side (13.10) equals

$$
\begin{aligned}
& \frac{1}{2^{6}} \iint\left[\frac{\partial \Omega}{\partial \xi_{1}}, \mu_{1}\right]\left[\bar{\mu}_{1}, \frac{\partial \Omega}{\partial \bar{\xi}_{1}^{\prime}}\right] \\
& \quad \times\left[\bar{\mu}_{1}, \mu_{1}\right]\left[\bar{\mu}_{2}, \mu_{2}\right]^{2} d^{2} \mu_{2} d^{2} \mu_{1}(d \bar{\mu})_{2} \\
&=\frac{1}{2^{3}} \iint \delta\left(\bar{\mu}_{2}\right)\left[\frac{\partial \Omega}{\partial \xi_{1}}, \mu_{1}\right]\left[\bar{\mu}_{1}, \frac{\partial \Omega}{\partial \bar{\xi}_{1}^{\prime}}\right] \\
& \quad \times\left[\bar{\mu}_{1}, \mu_{1}\right] d^{2} \mu_{1}(d \bar{\mu})_{2} \\
&=\frac{i^{2}}{2^{2}} \iint \delta\left(\bar{\mu}_{2}\right)\left[\bar{\mu}_{1}, \frac{\partial \Omega}{\partial \bar{\xi}_{1}^{\prime}}\right]\left\{\bar{\mu}_{1}, \frac{\partial \Omega}{\partial \xi_{1}}\right\} d^{2} \bar{\mu}_{1} d^{2} \bar{\mu}_{2} \\
&=-\frac{1}{2}\left[\frac{\partial \Omega\left(\bar{\xi}^{\prime}, \xi\right)}{\partial \xi_{1}}, \frac{\partial \Omega\left(\bar{\xi}^{\prime}, \xi\right)}{\partial \bar{\xi}_{1}^{\prime}}\right] .
\end{aligned}
$$

The integral with the second term in (13.10) is taking in a similar way and as a result, we reproduce the second term on the right-hand side (12.13).

\section{The star product}

It remains for us to compare the expression for the convolution (13.8) with the expression for the function $\tilde{\Omega}$, Eq. (12.4), which follows from the matrix element of the operator $a_{0}^{2}$. But we shall defer this comparison until the following section, and here we would like to introduce an important notion of a star product $*$ in a class of functions depending on the para-Grassmann variables. For this purpose we return to the connection between the functions $\widetilde{\Omega}$ and $\Omega$ as it was defined by the convolution integral, Eq. (12.5). We present the integrand as a result of action of the shift operators on $\Omega$ :

$$
\begin{aligned}
& \Omega\left(\bar{\xi}^{\prime}+\bar{\mu}, \xi\right)=\mathrm{e}^{\frac{1}{2}\left[\bar{\mu}, \vec{\partial} / \partial \bar{\xi}^{\prime}\right]} \Omega\left(\bar{\xi}^{\prime}, \xi\right), \\
& \Omega\left(\bar{\xi}^{\prime}, \xi+\mu\right)=\Omega\left(\bar{\xi}^{\prime}, \xi\right) \mathrm{e}^{-\frac{1}{2}[\mu, \overleftarrow{\partial} / \partial \xi]} .
\end{aligned}
$$

By a direct calculation, we verify that these expressions reproduce the expansions (12.6) and (12.7). To be specific, let us consider the first one

$$
\begin{aligned}
\mathrm{e}^{\frac{1}{2}\left[\bar{\mu}, \vec{\partial} / \partial \bar{\xi}^{\prime}\right]} \Omega\left(\bar{\xi}^{\prime}, \xi\right)= & \Omega\left(\bar{\xi}^{\prime}, \xi\right)+\frac{1}{2} \sum_{l=1}^{2}\left[\bar{\mu}_{l}, \frac{\partial \Omega}{\partial \bar{\xi}_{l}^{\prime}}\right] \\
& +\frac{1}{2 !}\left(\frac{1}{2}\right)^{2} \sum_{l=1}^{2} \sum_{s=1}^{2}\left[\bar{\mu}_{l}, \frac{\partial}{\partial \bar{\xi}_{l}^{\prime}}\right] \\
& \times\left[\bar{\mu}_{s}, \frac{\partial \Omega}{\partial \bar{\xi}_{s}^{\prime}}\right]
\end{aligned}
$$

The function $\Omega$ depends quadratically on the para-Grassmann variable $\bar{\xi}_{l}^{\prime}$, because of this, the expansion is exactly terminated on the third term. The first two terms coincides with the corresponding terms in (12.7), and so there is a need to analyze only the last term. Let us consider the contribution with $s=1$ and use an explicit form of the derivative $\partial \Omega / \partial \bar{\xi}_{1}^{\prime}$, Eq. (10.14), then

$$
\begin{aligned}
{\left[\bar{\mu}_{1}, \frac{\partial \Omega}{\partial \bar{\xi}_{1}^{\prime}}\right]=- } & \frac{1}{2^{2}}\left\{\left[\bar{\mu}_{1}, \bar{\xi}_{2}^{\prime}\right]\left[\xi_{1}, \xi_{2}\right]+\left[\bar{\mu}_{1}, \xi_{2}\right]\left[\bar{\xi}_{2}^{\prime}, \xi_{1}\right]\right. \\
& \left.-\left[\bar{\mu}_{1}, \xi_{1}\right]\left[\bar{\xi}_{2}^{\prime}, \xi_{2}\right]\right\}-\left[\bar{\mu}_{1}, \xi_{1}\right] .
\end{aligned}
$$

Under the action of the operator $\left[\bar{\mu}_{l}, \partial / \partial \bar{\xi}_{l}^{\prime}\right]$ on the last expression only the term with $l=2$ gives a nontrivial contribution. By employing the differentiation rules (C.1)-(C.4), further we find

$$
\begin{aligned}
{\left[\bar{\mu}_{2}, \frac{\partial}{\partial \bar{\xi}_{2}^{\prime}}\right]\left[\bar{\mu}_{1}, \frac{\partial \Omega}{\partial \bar{\xi}_{1}^{\prime}}\right] } \\
=-\frac{1}{2}\left\{\left[\bar{\mu}_{1}, \bar{\mu}_{2}\right]\left[\xi_{1}, \xi_{2}\right]+\left[\bar{\mu}_{1}, \xi_{2}\right]\left[\bar{\mu}_{2}, \xi_{1}\right]\right. \\
\left.\quad-\left[\bar{\mu}_{1}, \xi_{1}\right]\left[\bar{\mu}_{2}, \xi_{2}\right]\right\} .
\end{aligned}
$$

The second nontrivial contribution follows from the expression $\left[\bar{\mu}_{1}, \partial / \partial \bar{\xi}_{1}^{\prime}\right]\left[\bar{\mu}_{2}, \partial \Omega / \partial \bar{\xi}_{2}^{\prime}\right]$. By doing so we exactly reproduce the expression (12.7). 
The second expression in (14.1) is analysed similarly, but only here the rule of action of the right derivative on the function $\Omega$ must be taken into consideration:

$\Omega \frac{\overleftarrow{\partial}}{\partial \xi_{l}}=-\frac{\vec{\partial} \Omega}{\partial \xi_{l}}$

Based on the representation (14.1) we write the convolution (12.5) in the following form:

$$
\begin{aligned}
\widetilde{\Omega}\left(\bar{\xi}^{\prime}, \xi\right)= & \Omega\left(\bar{\xi}^{\prime}, \xi\right) \\
& \times\left[\iint \mathrm{e}^{-\frac{1}{2}[\mu, \overleftarrow{\partial} / \partial \xi]} \mathrm{e}^{-\frac{1}{2}[\bar{\mu}, \mu]}\right. \\
& \left.\times \mathrm{e}^{\frac{1}{2}\left[\bar{\mu}, \vec{\partial} / \partial \bar{\xi}^{\prime}\right]}(d \mu)_{2}(d \bar{\mu})_{2}\right] \Omega\left(\bar{\xi}^{\prime}, \xi\right)
\end{aligned}
$$

or somewhat differently

$$
\begin{aligned}
\widetilde{\Omega}\left(\bar{\xi}^{\prime}, \xi\right)= & \Omega\left(\bar{\xi}^{\prime}, \xi\right) \\
& \times\left[\iint \mathrm{e}^{-\frac{1}{2}\left[\bar{\mu}-\overleftarrow{\partial} / \partial \xi, \mu-\vec{\partial} / \partial \bar{\xi}^{\prime}\right]}(d \mu)_{2}(d \bar{\mu})_{2}\right] \\
& \times \mathrm{e}^{\frac{1}{2}\left[\overleftarrow{\partial} / \partial \xi, \vec{\partial} / \partial \bar{\xi}^{\prime}\right]} \Omega\left(\bar{\xi}^{\prime}, \xi\right)
\end{aligned}
$$

We take the following definition of the para-Grassmann star product:

$$
\begin{aligned}
& *=\iint \mathrm{e}^{-\frac{1}{2}[\mu, \overleftarrow{\partial} / \partial \xi]} \mathrm{e}^{-\frac{1}{2}[\bar{\mu}, \mu]} \mathrm{e}^{\frac{1}{2}\left[\bar{\mu}, \vec{\partial} / \partial \bar{\xi}^{\prime}\right]} \\
& \times(d \mu)_{2}(d \bar{\mu})_{2} \text {. }
\end{aligned}
$$

Using this definition we can proof isomorphism between the algebra of creation and annihilation operators $a_{n}^{ \pm}$obeying the para-Fermi statistics of order 2

$\check{a}_{i} \check{a}_{j} \check{a}_{k}+\check{a}_{k} \check{a}_{j} \check{a}_{i}=2 \check{\delta}_{i j} \check{a}_{k}+2 \check{\delta}_{k j} \check{a}_{i}$,

where the operator $\check{a}_{i}$ denotes $a_{i}^{+}$or $a_{i}^{-}$and $\check{\delta}_{i j}=\delta_{i j}$ when $\check{a}_{i}=a_{i}^{-}\left(a_{i}^{+}\right)$and $\check{a}_{j}=a_{j}^{+}\left(a_{j}^{-}\right)$; otherwise $\check{\delta}_{i j}=0$, and the algebra of para-Grassmann numbers of the same order equipped with the star product

$\check{\xi}_{i} * \check{\xi}_{j} * \check{\xi}_{k}+\check{\xi}_{k} * \check{\xi}_{j} * \check{\xi}_{i}=2 \check{\delta}_{i j} \check{\xi}_{k}+2 \check{\delta}_{k j} \check{\xi}_{i}$,

where the para-Grassmann numbers $\check{\xi}_{i}$ denote $\xi_{i}$ or $\bar{\xi}_{i}^{\prime}$. On the left-hand side of the last expression we have a triple star product and consequently it is of importance to ascertain that the star product (14.3) is associative. Details of the proof are presented in [63].

\section{Triple star product $\Omega * \Omega * \Omega$}

In terms of the definition of the star product (14.3) the integral convolution (12.5) is written in the form

$\widetilde{\Omega}\left(\bar{\xi}^{\prime}, \xi\right)=\Omega\left(\bar{\xi}^{\prime}, \xi\right) * \Omega\left(\bar{\xi}^{\prime}, \xi\right)$.
From the other hand, in Sect. 7 and in Appendix F by a direct calculation we have proved a validity of the relation

$\Omega\left(\bar{\xi}^{\prime}, \xi\right)=\Omega\left(\bar{\xi}^{\prime}, \xi\right) * \widetilde{\Omega}\left(\bar{\xi}^{\prime}, \xi\right)$.

Thus, if the relation (15.1) was indeed valid, then by virtue of (15.2) the following equality with triple star product would take place:

$\Omega\left(\bar{\xi}^{\prime}, \xi\right)=\Omega\left(\bar{\xi}^{\prime}, \xi\right) * \Omega\left(\bar{\xi}^{\prime}, \xi\right) * \Omega\left(\bar{\xi}^{\prime}, \xi\right)$.

This expression is a classical analogue of the operator equality

$a_{0}^{3}=a_{0}$.

The consequence of this operator equality is a possibility to present the operator $\hat{A}$, in the second form $(7.1 \mathrm{~b})$, in the exponential form (see Eq. (3.6))

$\hat{A}=\alpha\left[\hat{I}-\left(\frac{i \sqrt{3}}{2}\right) a_{0}-\frac{3}{2}\left(a_{0}\right)^{2}\right] \equiv \alpha \mathrm{e}^{-i \frac{2 \pi}{3} a_{0}}$.

The matrix element of this operator as it was defined by the expression (10.23) in view of (15.1) and (15.3) also can be presented in the compact form:

$\left\langle\bar{\xi}^{\prime}|\hat{A}| \xi\right\rangle=\mathcal{A}\left(\bar{\xi}^{\prime}, \xi\right)\left\langle\bar{\xi}^{\prime} \mid \xi\right\rangle$,

where

$$
\begin{aligned}
\mathcal{A}\left(\bar{\xi}^{\prime}, \xi\right) & =\alpha\left[I-\left(\frac{i \sqrt{3}}{2}\right) \Omega-\frac{3}{2} \Omega * \Omega\right] \\
& \equiv \alpha \mathrm{e}_{*}^{-i \frac{2 \pi}{3} \Omega\left(\bar{\xi}^{\prime}, \xi\right)} .
\end{aligned}
$$

Here,

$\mathrm{e}_{*}^{x}=\sum_{k=0}^{\infty} \frac{1}{k !}(x)_{*}^{k}, \quad(x)_{*}^{k} \equiv \underbrace{(x * x * \ldots * x)}_{k \text { times }}$

is the star exponential [32]. Thus, we only need to verify a validity of (15.1). In our reasoning we will follow consideration of Appendix F.

We make use of the notations from Sect. 5

$x \equiv \frac{1}{2}\left[\bar{\xi}_{1}^{\prime}, \xi_{1}\right], \quad y \equiv \frac{1}{2}\left[\bar{\xi}_{2}^{\prime}, \xi_{2}\right]$

and present the function $\Omega$ in the form

$\Omega\left(\bar{\xi}^{\prime}, \xi\right)=\Delta \Omega\left(\bar{\xi}^{\prime}, \xi\right)-(x+y-1)$,

where

$$
\begin{aligned}
\Delta \Omega\left(\bar{\xi}^{\prime}, \xi\right)= & -\frac{1}{2^{3}}\left[\bar{\xi}_{1}^{\prime}, \bar{\xi}_{2}^{\prime}\right]\left[\xi_{1}, \xi_{2}\right] \\
& -\frac{1}{2^{3}}\left[\bar{\xi}_{1}^{\prime}, \xi_{2}\right]\left[\bar{\xi}_{2}^{\prime}, \xi_{1}\right]+\frac{1}{2} x y .
\end{aligned}
$$

From here, in particular it follows that

$$
\begin{aligned}
{\left[\Omega\left(\bar{\xi}^{\prime}, \xi\right)\right]^{2}=} & \left(\Delta \Omega\left(\bar{\xi}^{\prime}, \xi\right)\right)^{2}-2(x+y-1) \Delta \Omega\left(\bar{\xi}^{\prime}, \xi\right) \\
& +(x+y-1)^{2} .
\end{aligned}
$$


Our purpose is to rewrite the expression obtained in Sects. 12 and 13 for the star product $\Omega * \Omega$, Eq. (13.8), in terms of the variables $x$ and $y$ with subsequent comparison with (12.4). To do so, we use the formulas obtained in Appendix F, Eq. (F.7)(F.10). On the strength of these formulas for the term in (15.6) linear in $\Delta \Omega$ we have

$$
\begin{gathered}
(x+y-1) \Delta \Omega\left(\bar{\xi}^{\prime}, \xi\right)=\frac{1}{2^{3}}\left(\left[\bar{\xi}_{1}^{\prime}, \bar{\xi}_{2}^{\prime}\right]\left[\xi_{1}, \xi_{2}\right]\right. \\
\left.+\left[\bar{\xi}_{1}^{\prime}, \xi_{2}\right]\left[\bar{\xi}_{2}^{\prime}, \xi_{1}\right]\right)-\frac{1}{2} x y+(x+y) x y .
\end{gathered}
$$

For the term in (15.6) quadratic in $\Delta \Omega$ the same formulas lead to the following expression:

$$
\begin{aligned}
(\Delta \Omega & \left.\left(\bar{\xi}^{\prime}, \xi\right)\right)^{2}=\frac{1}{2^{6}}\left[\bar{\xi}_{1}^{\prime}, \bar{\xi}_{2}^{\prime}\right]^{2}\left[\xi_{1}, \xi_{2}\right]^{2} \\
& +\frac{1}{2^{6}}\left[\bar{\xi}_{1}^{\prime}, \xi_{2}\right]^{2}\left[\bar{\xi}_{2}^{\prime}, \xi_{1}\right]^{2}+\frac{1}{2^{2}} x^{2} y^{2} \\
& +\frac{1}{2^{5}}\left[\bar{\xi}_{1}^{\prime}, \bar{\xi}_{2}^{\prime}\right]\left[\xi_{1}, \xi_{2}\right]\left[\bar{\xi}_{1}^{\prime}, \xi_{2}\right]\left[\bar{\xi}_{2}^{\prime}, \xi_{1}\right] \\
& -\frac{1}{2^{3}}\left[\bar{\xi}_{1}^{\prime}, \bar{\xi}_{2}^{\prime}\right]\left[\xi_{1}, \xi_{2}\right] x y \\
& -\frac{1}{2^{3}}\left[\bar{\xi}_{1}^{\prime}, \xi_{2}\right]\left[\bar{\xi}_{2}^{\prime}, \xi_{1}\right] x y .
\end{aligned}
$$

In view of (F.10) the last two terms here equal $x^{2} y^{2} / 4$. Let us consider the first term. By algebra of para-Grassmann numbers of order $p=2$, Eq. (B.2), this term can be presented as follows:

$$
\begin{aligned}
& {\left[\bar{\xi}_{1}^{\prime}, \bar{\xi}_{2}^{\prime}\right]^{2}\left[\xi_{1}, \xi_{2}\right]^{2}} \\
& \quad=\left(\bar{\xi}_{1}^{\prime} \bar{\xi}_{2}^{\prime} \bar{\xi}_{2}^{\prime} \bar{\xi}_{1}^{\prime}+\bar{\xi}_{2}^{\prime} \bar{\xi}_{1}^{\prime} \bar{\xi}_{1}^{\prime} \bar{\xi}_{2}^{\prime}\right)\left(\xi_{1} \xi_{2} \xi_{2} \xi_{1}+\xi_{2} \xi_{1} \xi_{1} \xi_{2}\right) \\
& \quad=2^{2}\left(\bar{\xi}_{1}^{\prime}\right)^{2}\left(\bar{\xi}_{2}^{\prime}\right)^{2}\left(\xi_{1}\right)^{2}\left(\xi_{2}\right)^{2}=2^{2}\left(\bar{\xi}_{1}^{\prime}\right)^{2}\left(\xi_{1}\right)^{2}\left(\bar{\xi}_{2}^{\prime}\right)^{2}\left(\xi_{2}\right)^{2} \\
& \quad=\left[\bar{\xi}_{1}^{\prime}, \xi_{1}\right]^{2}\left[\bar{\xi}_{2}^{\prime}, \xi_{2}\right]^{2}=2^{4} x^{2} y^{2} .
\end{aligned}
$$

Here, at the last step we have used Eqs. (F.7) and (F.8). Similar reasoning for the second term in (15.7) results in the equality $\left[\bar{\xi}_{1}^{\prime}, \xi_{2}\right]^{2}\left[\bar{\xi}_{2}^{\prime}, \xi_{1}\right]^{2}=2^{4} x^{2} y^{2}$.

We need only to consider the mixed contribution to (15.7), which we present as a product of two multiplies

$$
\left(\left[\bar{\xi}_{1}^{\prime}, \bar{\xi}_{2}^{\prime}\right]\left[\bar{\xi}_{1}^{\prime}, \xi_{2}\right]\right)\left(\left[\xi_{1}, \xi_{2}\right]\left[\bar{\xi}_{2}^{\prime}, \xi_{1}\right]\right) \text {. }
$$

For the first factor, by virtue of algebra (B.2), we have

$$
\begin{aligned}
{\left[\bar{\xi}_{1}^{\prime}, \bar{\xi}_{2}^{\prime}\right]\left[\bar{\xi}_{1}^{\prime}, \xi_{2}\right] } & =-\bar{\xi}_{1}^{\prime} \bar{\xi}_{2}^{\prime} \xi_{2} \bar{\xi}_{1}^{\prime}-\bar{\xi}_{2}^{\prime} \bar{\xi}_{1}^{\prime} \bar{\xi}_{1}^{\prime} \xi_{2} \\
& =\left(\bar{\xi}_{1}^{\prime}\right)^{2}\left(\xi_{2} \bar{\xi}_{2}^{\prime}+\bar{\xi}_{2}^{\prime} \xi_{2}\right)
\end{aligned}
$$

and for the second one, correspondingly, we get

$$
\begin{aligned}
{\left[\xi_{1}, \xi_{2}\right]\left[\bar{\xi}_{2}^{\prime}, \xi_{1}\right] } & =\xi_{1} \xi_{2} \bar{\xi}_{2}^{\prime} \xi_{1}+\xi_{2} \xi_{1} \xi_{1} \bar{\xi}_{2}^{\prime} \\
& =-\left(\xi_{1}\right)^{2}\left(\xi_{2} \bar{\xi}_{2}^{\prime}+\bar{\xi}_{2}^{\prime} \xi_{2}\right) .
\end{aligned}
$$

Their multiplication gives us the required expression

$$
\left[\bar{\xi}_{1}^{\prime}, \bar{\xi}_{2}^{\prime}\right]\left[\xi_{1}, \xi_{2}\right]\left[\bar{\xi}_{1}^{\prime}, \xi_{2}\right]\left[\bar{\xi}_{2}^{\prime}, \xi_{1}\right]=2\left(\bar{\xi}_{1}^{\prime}\right)^{2}\left(\xi_{1}\right)^{2}\left(\bar{\xi}_{2}^{\prime}\right)^{2}\left(\xi_{2}\right)^{2}
$$

$$
\begin{aligned}
& =\frac{1}{2}\left[\bar{\xi}_{1}^{\prime}, \xi_{1}\right]^{2}\left[\bar{\xi}_{2}^{\prime}, \xi_{2}\right]^{2} \\
& =2^{3} x^{2} y^{2} .
\end{aligned}
$$

Taking into consideration the aforementioned, we can write the expression (15.7) in a very simple form:

$\left(\Delta \Omega\left(\bar{\xi}^{\prime}, \xi\right)\right)^{2}=\frac{3}{2} x^{2} y^{2}$.

Therefore, the square of the function $\Omega$, Eq. (15.6), can be written in the following form:

$$
\begin{aligned}
{\left[\Omega\left(\bar{\xi}^{\prime}, \xi\right)\right]^{2}=} & \frac{3}{2} x^{2} y^{2}-2\left(x^{2} y+x y^{2}\right)+3 x y \\
& +\left(x^{2}+y^{2}\right)-2(x+y)-2 \\
& -\frac{1}{2^{2}}\left(\left[\bar{\xi}_{1}^{\prime}, \bar{\xi}_{2}^{\prime}\right]\left[\xi_{1}, \xi_{2}\right]+\left[\bar{\xi}_{1}^{\prime}, \xi_{2}\right]\left[\bar{\xi}_{2}^{\prime}, \xi_{1}\right]\right) .
\end{aligned}
$$

Now we turn to the consideration of the term with the derivatives in (13.8). To be specific, let us consider a contribution of the form

$-\frac{1}{2}\left[\frac{\partial \Omega\left(\bar{\xi}^{\prime}, \xi\right)}{\partial \xi_{1}}, \frac{\partial \Omega\left(\bar{\xi}^{\prime}, \xi\right)}{\partial \bar{\xi}_{1}^{\prime}}\right]$.

Here, the derivations are defined by the general expressions (10.11) and (10.14) and equal

$$
\begin{aligned}
& \frac{\partial \Omega\left(\bar{\xi}^{\prime}, \xi\right)}{\partial \xi_{1}} \\
& \quad=-\frac{1}{2^{2}}\left(\left[\bar{\xi}_{1}^{\prime}, \bar{\xi}_{2}^{\prime}\right] \xi_{2}-\bar{\xi}_{2}^{\prime}\left[\bar{\xi}_{1}^{\prime}, \xi_{2}\right]+\bar{\xi}_{1}^{\prime}\left[\bar{\xi}_{2}^{\prime}, \xi_{2}\right]\right)+\bar{\xi}_{1}^{\prime}, \\
& \frac{\partial \Omega\left(\bar{\xi}^{\prime}, \xi\right)}{\partial \bar{\xi}_{1}^{\prime}} \\
& =-\frac{1}{2^{2}}\left(\bar{\xi}_{2}^{\prime}\left[\xi_{1}, \xi_{2}\right]+\left[\bar{\xi}_{2}^{\prime}, \xi_{1}\right] \xi_{2}-\left[\bar{\xi}_{2}^{\prime}, \xi_{2}\right] \xi_{1}\right)-\xi_{1} .
\end{aligned}
$$

The substitution of these derivatives into the commutator (15.9) after transformations similar previous ones, results in the following expression:

$$
\begin{aligned}
- & \frac{1}{2}\left[\frac{\partial \Omega\left(\bar{\xi}^{\prime}, \xi\right)}{\partial \xi_{1}}, \frac{\partial \Omega\left(\bar{\xi}^{\prime}, \xi\right)}{\partial \bar{\xi}_{1}^{\prime}}\right] \\
= & -\frac{1}{2} x y^{2}+x y-x \\
& -\frac{1}{2^{2}}\left(\left[\bar{\xi}_{1}^{\prime}, \bar{\xi}_{2}^{\prime}\right]\left[\xi_{1}, \xi_{2}\right]+\left[\bar{\xi}_{1}^{\prime}, \xi_{2}\right]\left[\bar{\xi}_{2}^{\prime}, \xi_{1}\right]\right) .
\end{aligned}
$$

The second commutator additional to (15.9) with the derivatives with respect to $\xi_{2}$ and $\bar{\xi}_{2}^{\prime}$ is obtained from this expression by the replacement $x \rightleftarrows y$ and then the term with derivatives in (13.8) takes its final form:

$$
\begin{aligned}
- & \frac{1}{2}\left[\frac{\partial \Omega\left(\bar{\xi}^{\prime}, \xi\right)}{\partial \xi}, \frac{\partial \Omega\left(\bar{\xi}^{\prime}, \xi\right)}{\partial \bar{\xi}^{\prime}}\right] \\
= & \frac{1}{2}\left(x^{2} y+x y^{2}\right)-2 x y+(x+y) \\
& +\frac{1}{2}\left(\left[\bar{\xi}_{1}^{\prime}, \bar{\xi}_{2}^{\prime}\right]\left[\xi_{1}, \xi_{2}\right]+\left[\bar{\xi}_{1}^{\prime}, \xi_{2}\right]\left[\bar{\xi}_{2}^{\prime}, \xi_{1}\right]\right) .
\end{aligned}
$$


Substituting the obtained expressions (15.8) and (15.10) into (13.8) and collecting similar terms, we derive an explicit form of the convolution $\Omega * \Omega$ :

$$
\begin{aligned}
& \Omega\left(\bar{\xi}^{\prime}, \xi\right) * \Omega\left(\bar{\xi}^{\prime}, \xi\right) \\
&= \frac{3}{2} x^{2} y^{2}-\frac{3}{2}\left(x^{2} y+x y^{2}\right)+\frac{3}{2} x y \\
&+\left(x^{2}+y^{2}\right)-(x+y)+1 \\
&-\frac{1}{2^{3}}\left(\left[\bar{\xi}_{1}^{\prime}, \bar{\xi}_{2}^{\prime}\right]\left[\xi_{1}, \xi_{2}\right]-\left[\bar{\xi}_{1}^{\prime}, \xi_{2}\right]\left[\bar{\xi}_{2}^{\prime}, \xi_{1}\right]\right) .
\end{aligned}
$$

This expression should be compared with the function $\widetilde{\Omega}\left(\bar{\xi}^{\prime}, \xi\right)$ as it is defined by Eq. (12.4). In terms of the notations (15.5) this function has the following form:

$$
\begin{aligned}
\widetilde{\Omega}\left(\bar{\xi}^{\prime}, \xi\right)= & 2 x^{2} y^{2}-2\left(x^{2} y+x y^{2}\right)+2 x y \\
& +\left(x^{2}+y^{2}\right)-(x+y)+1 .
\end{aligned}
$$

In spite of very close similarity of these two expressions, there is no coincidence in a literal sense, and thus the equality (15.1) does not take place. It is a powerful argument to the conclusion that the Geyer operator $a_{0}^{2}$ as it was originally defined by Eq. (2.17) should be considered simply as a symbol. It is not the result of the multiplication of two operators $a_{0} \cdot a_{0}$. In the next sections we will analyses this problem in more detail.

\section{Connection between the operators $\hat{\omega}^{2}$ and $a_{0}^{2}$ revised}

In Sect. 8 we have defined a connection between the HarishChandra and Geyer operators $\hat{\omega}^{2}$ and $a_{0}^{2}$. In establishing the connection between these two operators, we used the matrix relation derived by Harish-Chandra [6], namely (see Appendix A)

$B=3-\omega^{2}$,

which enables one to put the operator $\hat{\omega}^{2}$ in the following form:

$\hat{\omega}^{2}=\frac{1}{2}\left(1+\widehat{\eta}_{5}\right)$.

A particular consequence of this representation is the connection

$\hat{\omega}^{2}=a_{0}^{2}$.

However, as it was shown in the previous section this relation results in a contradiction. It should be particularly emphasized that the operator $\hat{\omega}^{2}$ on the left-hand side of (16.3) represents the square of the operator $\hat{\omega}$ as it was defined by Eq. (6.18), and the operator $a_{0}^{2}$ on the right-hand side was introduced into consideration by Geyer [22]. The latter as it will be clear from the subsequent discussion, should be considered simply as a symbol, but not the square of a certain operator.

Let us give up the relation (16.1) and consider what would be the consequences of this. For that purpose, we return to the formula (8.4). On the left-hand side of this formula we rewrite the second term in the operator representation:

$$
\begin{array}{r}
\widehat{\eta}_{\mu_{1}} \widehat{\eta}_{\mu_{2}}+\widehat{\eta}_{\mu_{1}} \widehat{\eta}_{\mu_{3}}+\widehat{\eta}_{\mu_{1}} \widehat{\eta}_{\mu_{4}}+\widehat{\eta}_{\mu_{2}} \widehat{\eta}_{\mu_{3}}+\widehat{\eta}_{\mu_{2}} \widehat{\eta}_{\mu_{4}}+\widehat{\eta}_{\mu_{3}} \widehat{\eta}_{\mu_{4}} \\
\quad=\left(\widehat{\eta}_{\mu_{1}} \widehat{\eta}_{\mu_{2}}+\widehat{\eta}_{\mu_{3}} \widehat{\eta}_{\mu_{4}}\right)+\left(\widehat{\eta}_{\mu_{1}}+\widehat{\eta}_{\mu_{2}}\right)\left(\widehat{\eta}_{\mu_{3}}+\widehat{\eta}_{\mu_{4}}\right) .(16.4)
\end{array}
$$

To be specific, we fix the values of the indices as follows: $\mu_{k} \equiv k, k=1,2,3,4$. The use of formulae (8.9) and (8.10) for the first term on the right-hand side in (16.4) gives us

$\widehat{\eta}_{1} \widehat{\eta}_{2}+\widehat{\eta}_{3} \widehat{\eta}_{4}=-2\left(N_{1}^{2}+N_{2}^{2}\right)+2$.

Further, by using the connection between the creation and annihilation operators $a_{k}^{ \pm}$and the operators $\hat{\beta}_{\mu}$, Eq. (4.3), we derive

$\hat{\beta}_{1}^{2}=\frac{1}{4}\left(\left(a_{1}^{+}\right)^{2}+\left(a_{1}^{-}\right)^{2}+\left\{a_{1}^{+}, a_{1}^{-}\right\}\right)$,
$\hat{\beta}_{2}^{2}=\frac{1}{4}\left(\left(a_{1}^{+}\right)^{2}+\left(a_{1}^{-}\right)^{2}-\left\{a_{1}^{+}, a_{1}^{-}\right\}\right)$.

Recalling the definition of $\eta$-matrices: $\eta_{\mu}=2 \beta_{\mu}^{2}-1$, we get further

$\widehat{\eta}_{1}+\widehat{\eta}_{2}=2\left(\hat{\beta}_{1}^{2}+\hat{\beta}_{2}^{2}\right)-2=\left\{a_{1}^{+}, a_{1}^{-}\right\}-2$,

and similar we have

$\widehat{\eta}_{3}+\widehat{\eta}_{4}=2\left(\hat{\beta}_{3}^{2}+\hat{\beta}_{4}^{2}\right)-2=\left\{a_{2}^{+}, a_{2}^{-}\right\}-2$.

Substituting (16.5), (16.7) and (16.8) into (16.4), and then into (8.4) and finally into (8.3), instead of (16.3), we derive

$$
\begin{aligned}
\hat{\omega}^{2}= & \frac{3}{4} a_{0}^{2}+\frac{1}{4}\left[\left(N_{1}^{2}+N_{2}^{2}-1\right)-2\left(\frac{1}{2}\left\{a_{1}^{+}, a_{1}^{-}\right\}-1\right)\right. \\
& \left.\times\left(\frac{1}{2}\left\{a_{2}^{+}, a_{2}^{-}\right\}-1\right)\right] .
\end{aligned}
$$

Here, we have taken into account the connection between the operator $a_{0}^{2}$ and the operator $\widehat{\eta}_{5}$, Eq. (8.11).

Let us define a matrix element of the expression (16.9) in the basis of parafermion coherent states. The matrix element of the first term on the right-hand side is given by the expressions (12.3) and (12.4) (or (15.12)). The matrix elements of the operators $N_{1}^{2}$ and $N_{2}^{2}$ are defined by the formula (10.16) and in terms of the variables $x$ and $y$ have the form

$$
\begin{aligned}
\left\langle\bar{\xi}^{\prime}\left|N_{1}^{2}\right| \xi\right\rangle & =\left(x^{2}-x+1\right)\left\langle\bar{\xi}^{\prime} \mid \xi\right\rangle, \\
\left\langle\bar{\xi}^{\prime}\left|N_{2}^{2}\right| \xi\right\rangle & =\left(y^{2}-y+1\right)\left\langle\bar{\xi}^{\prime} \mid \xi\right\rangle .
\end{aligned}
$$

We have only to define the matrix element of the last term in (16.9). Towards this end, we will need the following formulas:

$$
\begin{aligned}
& a_{k}^{-} a_{n}^{+}|\xi\rangle=\left(2 \delta_{k n}+\xi_{k} a_{n}^{+}\right)|\xi\rangle, \\
& \left\langle\bar{\xi}^{\prime}\right| a_{n}^{-} a_{k}^{+}=\left\langle\bar{\xi}^{\prime}\right|\left(a_{n}^{-} \bar{\xi}_{k}^{\prime}+2 \delta_{k n}\right) .
\end{aligned}
$$


Then for the anticommutators $\left\{a_{k}^{+}, a_{k}^{-}\right\}$by virtue of these formulas we have

$$
\left\langle\bar{\xi}^{\prime}\left|\left\{a_{k}^{+}, a_{k}^{-}\right\}\right| \xi\right\rangle=\left(\left\{\bar{\xi}_{k}^{\prime}, \xi_{k}\right\}+2\right)\left\langle\bar{\xi}^{\prime} \mid \xi\right\rangle .
$$

Let us consider the matrix element of a product of two anticommutators, which we present as a sum of four terms

$$
\begin{aligned}
& \left\langle\bar{\xi}^{\prime}\left|\left\{a_{1}^{+}, a_{1}^{-}\right\}\left\{a_{2}^{+}, a_{2}^{-}\right\}\right| \xi\right\rangle \\
& =\left\langle\bar{\xi}^{\prime}\left|a_{1}^{+} a_{1}^{-} a_{2}^{+} a_{2}^{-}\right| \xi\right\rangle+\left\langle\bar{\xi}^{\prime}\left|a_{1}^{+} a_{1}^{-} a_{2}^{-} a_{2}^{+}\right| \xi\right\rangle \\
& \quad+\left\langle\bar{\xi}^{\prime}\left|a_{1}^{-} a_{1}^{+} a_{2}^{+} a_{2}^{-}\right| \xi\right\rangle+\left\langle\bar{\xi}^{\prime}\left|a_{1}^{-} a_{1}^{+} a_{2}^{-} a_{2}^{+}\right| \xi\right\rangle .
\end{aligned}
$$

For the first three terms, by using the relations (16.11) it is not difficult to obtain, correspondingly

$$
\begin{aligned}
& \left\langle\bar{\xi}^{\prime}\left|a_{1}^{+} a_{1}^{-} a_{2}^{+} a_{2}^{-}\right| \xi\right\rangle=\bar{\xi}_{1}^{\prime} \xi_{1} \bar{\xi}_{2}^{\prime} \xi_{2}\left\langle\bar{\xi}^{\prime} \mid \xi\right\rangle, \\
& \left\langle\bar{\xi}^{\prime}\left|a_{1}^{+} a_{1}^{-} a_{2}^{-} a_{2}^{+}\right| \xi\right\rangle=\left(-\bar{\xi}_{1}^{\prime} \bar{\xi}_{2}^{\prime} \xi_{2} \xi_{1}+2 \bar{\xi}_{1}^{\prime} \xi_{1}\right)\left\langle\bar{\xi}^{\prime} \mid \xi\right\rangle, \\
& \left\langle\bar{\xi}^{\prime}\left|a_{1}^{-} a_{1}^{+} a_{2}^{+} a_{2}^{-}\right| \xi\right\rangle=\left(-\bar{\xi}_{2}^{\prime} \bar{\xi}_{1}^{\prime} \xi_{1} \xi_{2}+2 \bar{\xi}_{2}^{\prime} \xi_{2}\right)\left\langle\bar{\xi}^{\prime} \mid \xi\right\rangle .
\end{aligned}
$$

The analysis of the last term in (16.13) is somewhat more complicated. Here, from (16.11) we have

$$
\begin{aligned}
& \left\langle\bar{\xi}^{\prime}\left|a_{1}^{-} a_{1}^{+} a_{2}^{-} a_{2}^{+}\right| \xi\right\rangle=\left\langle\bar{\xi}^{\prime}\left|\left(a_{1}^{-} \bar{\xi}_{1}^{\prime}+2\right)\left(\xi_{2} a_{2}^{+}+2\right)\right| \xi\right\rangle \\
& \quad=\left\langle\bar{\xi}^{\prime}\left|a_{1}^{-} \bar{\xi}_{1}^{\prime} \xi_{2} a_{2}^{+}\right| \xi\right\rangle+2\left(\xi_{1} \bar{\xi}_{1}^{\prime}+\xi_{2} \bar{\xi}_{2}^{\prime}\right)\left\langle\bar{\xi}^{\prime} \mid \xi\right\rangle+4\left\langle\bar{\xi}^{\prime} \mid \xi\right\rangle .
\end{aligned}
$$

We transform the expression in the first term by using the permutation rules (B.8)

$a_{1}^{-} \bar{\xi}_{1}^{\prime} \xi_{2} a_{2}^{+}=-a_{1}^{-} a_{2}^{+} \xi_{2} \bar{\xi}_{1}^{\prime}$,

then

$$
\left\langle\bar{\xi}^{\prime}\left|a_{1}^{-} \bar{\xi}_{1}^{\prime} \xi_{2} a_{2}^{+}\right| \xi\right\rangle=\xi_{2} \bar{\xi}_{2}^{\prime} \xi_{1} \bar{\xi}_{1}^{\prime}\left\langle\bar{\xi}^{\prime} \mid \xi\right\rangle .
$$

Taking into account the obtained expressions (16.12) and (16.13), we derive an explicit form of the matrix element of the last term in (16.9):

$$
\begin{aligned}
\left\langle\bar{\xi}^{\prime}\right. & \left.\left|\left(\frac{1}{2}\left\{a_{1}^{+}, a_{1}^{-}\right\}-1\right)\left(\frac{1}{2}\left\{a_{2}^{+}, a_{2}^{-}\right\}-1\right)\right| \xi\right\rangle \\
= & {\left[\frac { 1 } { 4 } \left(\bar{\xi}_{1}^{\prime} \xi_{1} \bar{\xi}_{2}^{\prime} \xi_{2}-\bar{\xi}_{1}^{\prime} \bar{\xi}_{2}^{\prime} \xi_{2} \xi_{1}-\bar{\xi}_{2}^{\prime} \bar{\xi}_{1}^{\prime} \xi_{1} \xi_{2}+\xi_{2} \bar{\xi}_{2}^{\prime} \xi_{1} \bar{\xi}_{1}^{\prime}\right.\right.} \\
& \left.+2\left\{\bar{\xi}_{1}^{\prime}, \xi_{1}\right\}+2\left\{\bar{\xi}_{2}^{\prime}, \xi_{2}\right\}+4\right) \\
& \left.-\frac{1}{2}\left(\left\{\bar{\xi}_{1}^{\prime}, \xi_{1}\right\}+\left\{\bar{\xi}_{2}^{\prime}, \xi_{2}\right\}\right)-2+1\right]\left\langle\bar{\xi}^{\prime} \mid \xi\right\rangle .
\end{aligned}
$$

As we can see from this expression, all terms of zeroth and second orders in the para-Grassmann variables are canceled out and thus, we eventually obtain

$$
\begin{aligned}
\left\langle\bar{\xi}^{\prime}\left|\left(\frac{1}{2}\left\{a_{1}^{+}, a_{1}^{-}\right\}-1\right)\left(\frac{1}{2}\left\{a_{2}^{+}, a_{2}^{-}\right\}-1\right)\right| \xi\right\rangle \\
=\frac{1}{4}\left(\bar{\xi}_{1}^{\prime} \xi_{1} \bar{\xi}_{2}^{\prime} \xi_{2}-\bar{\xi}_{1}^{\prime} \bar{\xi}_{2}^{\prime} \xi_{2} \xi_{1}-\bar{\xi}_{2}^{\prime} \bar{\xi}_{1}^{\prime} \xi_{1} \xi_{2}+\xi_{2} \bar{\xi}_{2}^{\prime} \xi_{1} \bar{\xi}_{1}^{\prime}\right) \\
\quad\left\langle\bar{\xi}^{\prime} \mid \xi\right\rangle .
\end{aligned}
$$

Making use of this expression and the formulas (15.12) and (16.10), we can now write the whole matrix element for the Harish-Chandra operator $\hat{\omega}^{2}$ in the representation (16.9)

$$
\left\langle\bar{\xi}^{\prime}\left|\hat{\omega}^{2}\right| \xi\right\rangle
$$

$$
\begin{aligned}
= & \left\{\frac{3}{2} x^{2} y^{2}-\frac{3}{2}\left(x^{2} y+x y^{2}\right)\right. \\
& +\frac{3}{2} x y+\left(x^{2}+y^{2}\right)-(x+y)+1-\frac{1}{2^{3}} \\
& \left.\times\left(\bar{\xi}_{1}^{\prime} \xi_{1} \bar{\xi}_{2}^{\prime} \xi_{1}-\bar{\xi}_{1}^{\prime} \bar{\xi}_{2}^{\prime} \xi_{2} \xi_{1}-\bar{\xi}_{2}^{\prime} \bar{\xi}_{1}^{\prime} \xi_{1} \xi_{2}+\xi_{2} \bar{\xi}_{2}^{\prime} \xi_{1} \bar{\xi}_{1}^{\prime}\right)\right\} \\
& \times\left\langle\bar{\xi}^{\prime} \mid \xi\right\rangle .
\end{aligned}
$$

If one takes into account the trivial identity

$$
\begin{aligned}
& {\left[\bar{\xi}_{1}^{\prime}, \bar{\xi}_{2}^{\prime}\right]\left[\xi_{1}, \xi_{2}\right]-\left[\bar{\xi}_{1}^{\prime}, \xi_{2}\right]\left[\bar{\xi}_{2}^{\prime}, \xi_{1}\right]} \\
& \quad=-\left(\bar{\xi}_{1}^{\prime} \xi_{1} \bar{\xi}_{2}^{\prime} \xi_{1}-\bar{\xi}_{1}^{\prime} \bar{\xi}_{2}^{\prime} \xi_{2} \xi_{1}-\bar{\xi}_{2}^{\prime} \bar{\xi}_{1}^{\prime} \xi_{1} \xi_{2}+\xi_{2} \bar{\xi}_{2}^{\prime} \xi_{1} \bar{\xi}_{1}^{\prime}\right),
\end{aligned}
$$

then we see that the expression (16.15) perfectly reproduces the expression for the convolution $\Omega * \Omega$, Eq. (15.11). In the subsequent sections we will discuss in more detail why omitting the Harish-Chandra relation (16.1) leads us to a correct result. It is precisely these expression (16.9) that defines the square of the operator $a_{0}(\equiv-\hat{\omega})$, which in contrast to the Geyer symbol $a_{0}^{2}$ will be designated as $\left(a_{0}\right)^{2}$.

\section{The Casimir operators $\widehat{C}_{2}$ and $\widehat{C}_{2}^{\prime}$}

In the paper Omote et al. [42] an explicit form of the quadratic Casimir operator for the group $S O(2 M)$ was written out in terms of the generators

$\widehat{C}_{2}=\sum_{k, l}\left(2 N_{k l} N_{l k}+L_{k l} M_{l k}+M_{k l} L_{l k}\right)$.

Here, the indices $k, l$ run $1,2, \ldots, M$. In our case $M=2$ and therefore

$\widehat{C}_{2}=2\left(-\left\{L_{12}, M_{12}\right\}+\left\{N_{12}, N_{21}\right\}+\left(N_{1}^{2}+N_{2}^{2}\right)\right)$.

For comparison, here we write out an explicit form of the operator $a_{0}$ obtained in Sect. 6

$a_{0}=-\frac{1}{4}\left(\left\{L_{12}, M_{12}\right\}+\left\{N_{12}, N_{21}\right\}-\left\{N_{1}, N_{2}\right\}\right)$.

For the group $S O(2 M+1)$ the quadratic Casimir operator is

$\widehat{C}_{2}^{\prime}=\widehat{C}_{2}+\hat{\Lambda}$,

where $\hat{\Lambda} \equiv \sum_{k=1}^{M}\left\{a_{k}^{+}, a_{k}^{-}\right\}$. In our case for $M=2$ the operator $\hat{\Lambda}$ is

$\hat{\Lambda}=\left\{a_{1}^{+}, a_{1}^{-}\right\}+\left\{a_{2}^{+}, a_{2}^{-}\right\}$.

From the form of Casimir's operator (17.1) we may notice that the contribution $\left(N_{1}^{2}+N_{2}^{2}\right)$ has its analogue in the expression for the Harish-Chandra operator (16.9). This suggests that the product

$\left(\frac{1}{2}\left\{a_{1}^{+}, a_{1}^{-}\right\}-1\right)\left(\frac{1}{2}\left\{a_{2}^{+}, a_{2}^{-}\right\}-1\right)$ 
from (16.9) may have a connection with the difference

$$
\left\{N_{12}, N_{21}\right\}-\left\{L_{12}, M_{12}\right\}
$$

from the definition of the Casimir operator (17.1). We verify this assumption by a direct transformation of (17.5).

Let us consider the first term in (17.5). By the definitions of the generators $N_{12}$ and $N_{21}$

$N_{12}=\frac{1}{2}\left[a_{1}^{+}, a_{2}^{-}\right], \quad N_{21}=\frac{1}{2}\left[a_{2}^{+}, a_{1}^{-}\right]$

and of the algebra of para-Fermi operators of order $p=2$, Eqs. (4.4)-(4.6), we have

$$
\begin{aligned}
N_{12} N_{21}= & \frac{1}{4}\left[-a_{1}^{+} a_{1}^{-} a_{2}^{+} a_{2}^{-}-a_{1}^{+} a_{2}^{-} a_{1}^{-} a_{2}^{+}-a_{2}^{-} a_{1}^{+} a_{2}^{+} a_{1}^{-}\right. \\
& \left.-a_{1}^{-} a_{1}^{+} a_{2}^{-} a_{2}^{+}+2\left(a_{1}^{+} a_{1}^{-}+a_{2}^{-} a_{2}^{+}\right)\right], \\
N_{21} N_{12}= & \frac{1}{4}\left[-a_{1}^{+} a_{1}^{-} a_{2}^{+} a_{2}^{-}-a_{1}^{+} a_{2}^{-} a_{1}^{-} a_{2}^{+}-a_{2}^{-} a_{1}^{+} a_{2}^{+} a_{1}^{-}\right. \\
& \left.-a_{1}^{-} a_{1}^{+} a_{2}^{-} a_{2}^{+}+2\left(a_{1}^{-} a_{1}^{+}+a_{2}^{+} a_{2}^{-}\right)\right] .
\end{aligned}
$$

Summing these two expressions, we get

$$
\begin{aligned}
& \left\{N_{12}, N_{21}\right\} \\
& =-\frac{1}{2}\left[a_{1}^{+} a_{1}^{-} a_{2}^{+} a_{2}^{-}+a_{1}^{+} a_{2}^{-} a_{1}^{-} a_{2}^{+}+a_{2}^{-} a_{1}^{+} a_{2}^{+} a_{1}^{-}\right. \\
& \left.\quad+a_{1}^{-} a_{1}^{+} a_{2}^{-} a_{2}^{+}-\left(\left\{a_{1}^{+}, a_{1}^{-}\right\}+\left\{a_{2}^{+}, a_{2}^{-}\right\}\right)\right] .
\end{aligned}
$$

In what follows we shall analyze the second term in (17.5). Here, we recall that the generators $L_{12}$ and $M_{12}$ are given by the expressions

$L_{12}=\frac{1}{2}\left[a_{1}^{+}, a_{2}^{+}\right], \quad M_{12}=\frac{1}{2}\left[a_{1}^{-}, a_{2}^{-}\right]$.

By virtue of the algebra (4.4)-(4.6), we have

$$
\begin{aligned}
L_{12} M_{12}= & \frac{1}{4}\left[-a_{1}^{+} a_{2}^{-} a_{1}^{-} a_{2}^{+}+a_{1}^{+} a_{1}^{-} a_{2}^{-} a_{2}^{+}+a_{1}^{-} a_{1}^{+} a_{2}^{+} a_{2}^{-}\right. \\
& \left.-a_{2}^{-} a_{1}^{+} a_{2}^{+} a_{1}^{-}-2\left(a_{1}^{+} a_{1}^{-}+a_{2}^{+} a_{2}^{-}\right)\right], \\
M_{12} L_{12}= & \frac{1}{4}\left[-a_{1}^{+} a_{2}^{-} a_{1}^{-} a_{2}^{+}+a_{1}^{+} a_{1}^{-} a_{2}^{-} a_{2}^{+}+a_{1}^{-} a_{1}^{+} a_{2}^{+} a_{2}^{-}\right. \\
& \left.-a_{2}^{-} a_{1}^{+} a_{2}^{+} a_{1}^{-}-2\left(a_{1}^{-} a_{1}^{+}+a_{2}^{-} a_{2}^{+}\right)\right],
\end{aligned}
$$

that in turn, gives

$$
\begin{aligned}
& \left\{L_{12}, M_{12}\right\} \\
& \quad=-\frac{1}{2}\left[a_{1}^{+} a_{2}^{-} a_{1}^{-} a_{2}^{+}-a_{1}^{+} a_{1}^{-} a_{2}^{-} a_{2}^{+}-a_{1}^{-} a_{1}^{+} a_{2}^{+} a_{2}^{-}\right. \\
& \left.\quad+a_{2}^{-} a_{1}^{+} a_{2}^{+} a_{1}^{-}+\left(\left\{a_{1}^{+}, a_{1}^{-}\right\}+\left\{a_{2}^{+}, a_{2}^{-}\right\}\right)\right] .
\end{aligned}
$$

Substituting this expression and (17.6) into (17.5), further we obtain

$$
\begin{aligned}
\left\{N_{12},\right. & \left.N_{21}\right\}-\left\{L_{12}, M_{12}\right\} \\
= & -\frac{1}{2}\left[a_{1}^{+} a_{1}^{-} a_{2}^{+} a_{2}^{-}+a_{1}^{-} a_{1}^{+} a_{2}^{-} a_{2}^{+}+a_{1}^{+} a_{1}^{-} a_{2}^{-} a_{2}^{+}\right. \\
& \left.+a_{1}^{-} a_{1}^{+} a_{2}^{+} a_{2}^{-}-2\left(\left\{a_{1}^{+}, a_{1}^{-}\right\}+\left\{a_{2}^{+}, a_{2}^{-}\right\}\right)\right] \\
= & -\frac{1}{2}\left[\left\{a_{1}^{+}, a_{1}^{-}\right\}\left\{a_{2}^{+}, a_{2}^{-}\right\}-2\left\{a_{1}^{+}, a_{1}^{-}\right\}-2\left\{a_{2}^{+}, a_{2}^{-}\right\}\right] \\
= & -2\left(\frac{1}{2}\left\{a_{1}^{+}, a_{1}^{-}\right\}-1\right)\left(\frac{1}{2}\left\{a_{2}^{+}, a_{2}^{-}\right\}-1\right)+2 .
\end{aligned}
$$

Thus, we finally have

$$
\begin{aligned}
& \left(\frac{1}{2}\left\{a_{1}^{+}, a_{1}^{-}\right\}-1\right)\left(\frac{1}{2}\left\{a_{2}^{+}, a_{2}^{-}\right\}-1\right) \\
& =-\frac{1}{2}\left(\left\{N_{12}, N_{21}\right\}-\left\{L_{12}, M_{12}\right\}\right)+1 .
\end{aligned}
$$

With allowance made for the last relation and the definition (17.1), the Harish-Chandra operator (16.9) can be cast in a rather compact and visual form

$\hat{\omega}^{2}=\frac{3}{4} a_{0}^{2}+\frac{1}{8}\left(\widehat{C}_{2}-6\right)$

or making use of the representation for the $a_{0}^{2}$ in terms of the $G$-parity operator, Eq. (9.13), it can be also written as

$\hat{\omega}^{2}=\frac{3}{8}\left[1+(-1)^{n}\right]+\frac{1}{8}\left(\widehat{C}_{2}-6\right)$.

The Casimir operator $\hat{C}_{2}$ (and correspondingly, $\hat{C}_{2}^{\prime}$ ) can be presented as a polynomial of the operator $\hat{\Lambda}$. For this purpose, it is easy to use the formulas (8.3)-(8.6) without recourse to the relation (16.1). Based on these formulas we have

$\hat{\omega}^{2}=\frac{3}{4} a_{0}^{2}+\frac{1}{4^{3}}\left[-(2 \hat{B}-4)^{2}+4^{2}\right]$.

Here, the operator $\hat{B} \equiv \hat{\beta}_{\mu} \hat{\beta}_{\mu}$ by the relations of the (16.6) type and the definition (17.4) equals

$\hat{B}=\frac{1}{2} \hat{\Lambda}$.

Comparing the last terms in (17.7) and (17.8), we can easily find the required representation

$\widehat{C}_{2}=-\frac{1}{2} \hat{\Lambda}(\hat{\Lambda}-8)$,

and as a consequence of (17.3), we obtain

$\widehat{C}_{2}^{\prime}=-\frac{1}{2} \hat{\Lambda}(\hat{\Lambda}-10)$.

We write out the rules of action of the Casimir operator $\widehat{C}_{2}$ on the vector states (6.1). They can be easily obtained by calculations identical with those performed by us in Sect. 6 
for the operator $a_{0}$ :

$$
\begin{array}{ll}
\widehat{C}_{2}|0\rangle=8|0\rangle, & \\
\widehat{C}_{2}|1\rangle=6|1\rangle, & \widehat{C}_{2}|2\rangle=6|2\rangle, \\
\widehat{C}_{2}|11\rangle=8|11\rangle, & \widehat{C}_{2}|22\rangle=8|22\rangle, \\
\widehat{C}_{2}|12\rangle=8|12\rangle, & \widehat{C}_{2}|21\rangle=8|21\rangle, \\
\widehat{C}_{2}|112\rangle=6|112\rangle, & \widehat{C}_{2}|221\rangle=6|221\rangle, \\
\widehat{C}_{2}|1122\rangle=8|1122\rangle . &
\end{array}
$$

Making use of these rules ${ }^{5}$, it is not difficult to see that action of the Harish-Chandra operator $\hat{\omega}^{2}$ in the representation (17.7) on the state vectors is exactly the same as the rules of action of the Geyer operator $a_{0}^{2}$, Eq. (6.2). By this means in the usual Fock space these two operators are fully equivalent. This equivalence breaks down if we introduce the so-called generalized state-vector space [26]. We will discuss this fact in the next section, but here, in the remainder of this section we analysed some properties of the Casimir operator $\widehat{C}_{2}^{\prime}$.

Let us define a connection of the Casimir operator $\widehat{C}_{2}^{\prime}$ for the $S O(2 M+1)$ group (for $M=2$ ) with $\theta$-element of the center of the DKP-algebra [6]. The element of the center, $\theta$, in the matrix representation is expressed in terms of the matrix $B$ with the help of relation

$\theta=B(5-B)$.

In the operator representation we have the connection (17.9) and consequently the operator representation of $\theta$ takes the form

$\hat{\theta}=\frac{1}{4} \hat{\Lambda}(10-\hat{\Lambda})$.

Comparing this expression with (17.11), we derive the required relation

$\widehat{C}_{2}^{\prime}=2 \hat{\theta}$.

In accordance with the Harish-Chandra approach the matrix $\theta$ (for $D=4$ ) has the eigenvalue 6 and therefore by virtue of the fact that $\theta$ is in the center of the DKP-algebra, it must be put

$\theta=6 I \equiv \theta_{0}$,

5 The eigenvalues obtained in the right-hand side of (17.12) are in perfect agreement with the conclusions of Bracken and Green [43]. In notations of the authors the quadratic Casimir operator $\widehat{\sigma}_{2}(2 M)$ of the $S O(2 M)$ group (which should be considered as a subgroup of the $S O(2 M+1)$ group) has the following eigenvalues for arbitrary $M$ and $p$

$\widehat{\sigma}_{2}(2 M)=\left[p M\left(M+\frac{1}{2} p-1\right)-2 q^{\prime}\left(p-q^{\prime}\right)\right] \hat{I}$,

where $q^{\prime}=0,1, \ldots, p$. For our specific case $M=2$ and $p=2$, it immediately follows that

$\widehat{\sigma}_{2}(4)= \begin{cases}8, & \text { if } q^{\prime}=0,2 \\ 6, & \text { if } q^{\prime}=1\end{cases}$ where $I$ is the unit matrix. In an operator formalism it goes in the relation

$\hat{\theta}=6 \hat{I}$

where in turn $\hat{I}$ is the unit operator. As a consequence, in view of (17.14) the Casimir operator for $S O(5)$ group is equal to

$\widehat{C}_{2}^{\prime}=12 \hat{I}$.

In fact it is precisely this relation ${ }^{6}$ that is the primary source of disagreement between two expressions (15.11) and (15.12).

Let us consider action of the operator $\hat{C}_{2}^{\prime}=\hat{C}_{2}+\hat{\Lambda}$ on the vector states. Action of the operator $\hat{C}_{2}$ is defined by the formulas (17.12), and action of the operator $\hat{\Lambda}$ is easily defined from its definition (17.4):

$\hat{\Lambda}|0\rangle=4|0\rangle, \quad \hat{\Lambda}|1\rangle=6|1\rangle, \quad \hat{\Lambda}|11\rangle=4|11\rangle, \ldots$,

i.e. action of $\hat{\Lambda}$ is reduced to multiplying by 4 or 6 depending on evenness of the number of parafermions in a particular state. Then for the Casimir operator $\hat{C}_{2}^{\prime}=\hat{C}_{2}+\hat{\Lambda}$ we have $\widehat{C}_{2}^{\prime}|0\rangle=12|0\rangle, \quad \widehat{C}_{2}^{\prime}|1\rangle=12|1\rangle, \quad \widehat{C}_{2}^{\prime}|11\rangle=12|11\rangle, \ldots$,

in a perfect agreement with the formula (17.16).

Let us calculate a matrix element of the operator $\hat{C}_{2}^{\prime}$ in the basis of parafermion coherent states. It is easy to determine the matrix element for the Casimir operator $\hat{C}_{2}$ based on the expression (17.7). Taking into account that the matrix element of the Harish-Chandra operator $\hat{\omega}^{2}$ is defined by the expression (15.11), and the matrix element of the Geyer operator $a_{0}^{2}$ is defined by expression (15.12), from (17.7) we derive

$$
\left\langle\bar{\xi}^{\prime}\left|\widehat{C}_{2}\right| \xi\right\rangle=2\left[2 \left\{\left(\frac{1}{2}\left[\bar{\xi}_{1}^{\prime}, \xi_{2}\right]\right)\left(\frac{1}{2}\left[\bar{\xi}_{2}^{\prime}, \xi_{1}\right]\right)\right.\right.
$$

6 This relation is in agreement again with the conclusions of the paper [43]. The quadratic Casimir operator $\widehat{\sigma}_{2}(2 M+1)$ for arbitrary $M$ and $p$ has the following eigenvalue:

$\widehat{\sigma}_{2}(2 M+1)=p M\left(M+\frac{1}{2} p\right) \hat{I}$,

whence, for $M=2$ and $p=2$, it follows that

$\widehat{\sigma}_{2}(5)=12 \hat{I}$.

In addition we note also that the invariant $\hat{q}$ introduced in the paper [43] in analysis of the reduction of the orthogonal group $S O(2 M+1)$ with respect to the unitary group $U(M)$ is simply related to the operator $\hat{\Lambda}$. From a general formula [43]

$\widehat{\sigma}_{2}(2 M+1)-\widehat{\sigma}_{2}(2 M)=2 \hat{q}(p-\hat{q})+p M$,

in our specific case with allowance made for the representations (17.10) and (17.11) it follows that

$2 \hat{q}(2-\hat{q})+4=\hat{\Lambda}$.

By this means the invariant $\hat{q}$ cannot be expressed as a rational function of $\hat{\Lambda}$. 


$$
\begin{aligned}
& \left.-\left(\frac{1}{2}\left[\bar{\xi}_{1}^{\prime}, \bar{\xi}_{2}^{\prime}\right]\right)\left(\frac{1}{2}\left[\xi_{1}, \xi_{2}\right]\right)\right\} \\
& \left.+\left(x^{2}+y^{2}\right)-(x+y)+4\right]\left\langle\bar{\xi}^{\prime} \mid \xi\right\rangle .
\end{aligned}
$$

Incidentally, this expression can be obtained by a direct calculation starting from the definition (17.1) with the use of the results in Sect. 7. The matrix element of the operator $\hat{\Lambda}$ equals

$\left\langle\bar{\xi}^{\prime}|\hat{\Lambda}| \xi\right\rangle=\left(\left\{\bar{\xi}_{1}^{\prime}, \xi_{1}\right\}+\left\{\bar{\xi}_{2}^{\prime}, \xi_{2}\right\}+4\right)\left\langle\bar{\xi}^{\prime} \mid \xi\right\rangle$.

Adding the last two expressions, we obtain that in the basis of parafermion coherent states the following non-equality takes place

$\left\langle\bar{\xi}^{\prime}\left|\widehat{C}_{2}^{\prime}\right| \xi\right\rangle \neq 12\left\langle\bar{\xi}^{\prime} \mid \xi\right\rangle$,

and in such a manner the relation (17.16) is not true within the framework of generalized state-vector space.

\section{Ohnuki and Kamefuchi's generalized state-vector space}

As was noted for the first time by Ohnuki and Kamefuchi [26] "The introduction of para-Grassmann numbers into the framework of our theory naturally necessitates a corresponding generalization of the state-vector space $\mathfrak{A}$." This statevector space is usually spanned by state vectors such as

$\mathcal{M}\left(a_{i}^{+}, a_{j}^{+}, \cdots\right)|0\rangle$,

where $\mathcal{M}$ is a monomial in the creation operators $a_{k}^{+}$, which in our case is defined by the expressions (6.1). The use of the para-Grassmann numbers $\xi_{k}$ results in the fact that we have to allow such $\mathcal{M}^{\prime}$ s to contain as well $\xi^{\prime}$ s. We have already faced with this situation in Sect. 9 in analysis of the structure of the parafermion coherent state $|\xi\rangle$. There was shown that some terms of the expansion of the coherent state in powers of $\left[\xi, a^{+}\right]$under the action on the vacuum state in principle are not reduced to the expansion in the state vectors (6.1).

Instead of the usual state-vector space $\mathfrak{A}$ now we should consider a generalized state-vector space $\mathfrak{A}_{G}$, which is spanned by ket vectors such as

$\mathcal{M}\left(a_{i}^{+}, a_{j}^{+}, \ldots, \xi_{k}, \ldots\right)|0\rangle$.

By this means the Harish-Chandra operator (17.7) and the Geyer operator (2.17) are equivalent in the space $\mathfrak{A}$ and are not equivalent in the enlarged space $\mathfrak{A}_{G}$. The accounting this circumstance results in an appreciable complication of the matrix elements of the operator expressions containing the Geyer operator $a_{0}^{2}$.
In principle, the complication of this kind can be avoided if instead of the parafermion coherent state of the form

$|\xi\rangle=\mathrm{e}^{-\frac{1}{2} \sum_{l}\left[\xi_{l}, a_{l}^{+}\right]}|0\rangle$

one uses the coherent state admitting an expansion in the number basis as it takes place in the case of the usual Fermi (and Bose) statistics. An example of such a coherent state for the case of a single-mode parafermi system can be found in the paper by Jing and Nelson [64]. In this paper the paraFermi eigenstate of annihilation operator $a^{-}$is presented in the form of the expansion in the number basis

$|n\rangle=\frac{1}{\sqrt{\{n\} !}}\left(a^{+}\right)^{n}|0\rangle$,

such that $\hat{n}_{f}|n\rangle=n|n\rangle$, where the parafermi number operator is

$\hat{n}_{f}=\frac{1}{2}\left[a^{+}, a^{-}\right]+\frac{1}{2} p$

and

$$
\begin{aligned}
\{n\} & \equiv n(p+1-n), \quad\{n\} !=\{n\}\{n-1\} \ldots\{1\} \\
= & \frac{n ! p !}{(p-n) !} .
\end{aligned}
$$

Note that the coefficient $\{n\}$ coincides with the coefficient in the differentiation formula of para-Grassmann number $\xi^{n}$, Eq. (C.3). The para-Fermi coherent state has the following form:

$\left|(\xi)_{p}\right\rangle=\sum_{n=0}^{p}|n\rangle \frac{\xi^{n}}{\sqrt{\{n\} !}}$.

It obeys the relation $a^{-}\left|(\xi)_{p}\right\rangle=\left|(\xi)_{p}\right\rangle \xi$ and has the overlap function

$\left\langle\left(\bar{\xi}^{\prime}\right)_{p} \mid(\xi)_{p}\right\rangle=\sum_{n=0}^{p} \frac{1}{\{n\} !}\left(\bar{\xi}^{\prime}\right)^{n}(\xi)^{n}$.

In particular, for the special case $p=2$ the expression (18.1) takes the form

$\left|(\xi)_{2}\right\rangle=|0\rangle+\frac{1}{\sqrt{2}}|1\rangle \xi+\frac{1}{2}|2\rangle \xi^{2}$.

Making use of the parafermion state (18.1) (and its generalization to two-mode para-Fermi system) enables one to avoid the introduction of the generalized state-vector space $\mathfrak{A}_{G}$, however, in so doing the usual exponential representation both of the coherent state and, correspondingly of the overlap function rather convenient for the construction of the path integral representation, is lost. 


\section{Another representation of Harish-Chandra operator $\hat{\omega}^{2}$}

We define the expression for Harish-Chandra operator $\hat{\omega}^{2}$, Eq. (17.7), in terms of the Geyer operator $a_{0}^{2}$ and the Casimir operator $\hat{C}_{2}$ for the orthogonal $S O(4)$ group. Let us define a representation for this operator in terms of the Casimir operator $\hat{C}_{2}^{\prime}$ for the $S O(5)$ group and the operator $\hat{\Lambda}$. For this purpose, we will need the formula (68) from the paper [6]. In the special case $M=2$ this formula (in the matrix representation) for the non-normalized matrix $\omega^{2}$ gives us

$\omega^{2}=4\left\{2 B_{2}-2 B_{3}+B_{4}\right\}$,

where the matrices $B_{k}$ are recurrently defined by the matrix $B$ (see Eq. (H.8))

$B_{2}=B(B-1), \quad B_{3}=B_{2}(B-2), \quad B_{4}=B_{3}(B-3)$.

It should be noted that the formula (68) in [6] was given with wrong number coefficients of the matrices $B_{k}$. In particular, for the case $M=2$ the coefficient 2 of $B_{2}$ is absent. We give the correct expression in (19.1). The formula (68) in HarishChandra's original text further is not used with the exception of the following formula (69) and therefore all subsequent expressions are true. However, the following formula (69) from [6] will be incorrect if one uses (68). We discuss in detail all of these questions in Appendix $\mathrm{H}$, where a correct expression for (68) is given.

Let us use the definition of the element of the center $\theta$, Eq. (17.13), to exclude in (19.2) all terms nonlinear in the matrix $B$, then

$$
\begin{aligned}
& B_{2}=-\theta+4 B, \\
& B_{3}=-2 \theta-B(\theta-12), \\
& B_{4}=\theta^{2}-6 \theta-B(4 \theta-24) .
\end{aligned}
$$

Putting these expressions into (19.1) yields

$\omega^{2}=4[P(\theta)+Q(\theta) B]$,

where $P(\theta) \equiv \theta^{2}-4 \theta$ and $Q(\theta) \equiv-2(\theta-4)$. We eliminate the term $\theta^{2}$ from the function $P(\theta)$ using the minimal equation for $\theta$ :

$(\theta-4)(\theta-6)=0$,

then $P(\theta)=6(\theta-4)$, and the expression (19.3) takes the form

$\omega^{2}=\frac{1}{2}(\theta-4)(3-B)$.

Here, we went to the normalized pseudo-matrix $\omega$ in accordance with the rule: $\omega \rightarrow 4 \omega$. The expression (19.4) correctly reproduces the formula (70) from [6] suggested by Harish-Chandra from general reasoning. In particular, when we fix the element of the center $\theta$, Eq. (17.15), from (19.4) follows (16.1). The use of the original formula (68) (see (H.2) in Appendix H) leads to the improper expressions (19.4) and (16.1).

For the operator formulation of the relation (19.4) we make use of the formulas of connection (17.14) and (17.9) that give us the desired representation for the Harish-Chandra operator

$\hat{\omega}^{2}=\frac{1}{8}\left(\widehat{C}_{2}^{\prime}-8\right)(6-\hat{\Lambda})$.

If one recalls the representation of the Casimir operator $\hat{C}_{2}^{\prime}$ in terms of $\hat{\Lambda}$, Eq. (17.11), then the preceding expression can be put into another form

$\hat{\omega}^{2}=\frac{1}{16}(\hat{\Lambda}-8)(\hat{\Lambda}-6)(\hat{\Lambda}-2)$

and thus the operator $\hat{\omega}^{2}$ represents a polynomial of the third order in $\hat{\Lambda}$. As a consequence of (19.5), (17.7) and (17.10), we obtain a similar representation for the Geyer operator $a_{0}^{2}$ :

$a_{0}^{2}=\frac{1}{12}(\hat{\Lambda}-7)(\hat{\Lambda}-6)(\hat{\Lambda}-2)$.

By this means, for the $a_{0}^{2}$ operator we have the third representation in this case in terms of $\hat{\Lambda}$. The first representation is the original expression of Geyer, Eq. (2.17), and the second one was written out in terms of the fermion number counter $(-1)^{n}$, Eq. (9.13).

By virtue of the obtained expressions (17.9)-(17.11), (19.5) and (19.6), one can state a question on the possibility of the representation of the operator $\hat{\omega}\left(\equiv-a_{0}\right)$ as a function of $\hat{\Lambda}$. However, analysis showed that the representation of the $\hat{\omega}$ as a polynomial of the second and the third orders in $\hat{\Lambda}$ is incompatible with the property

$\hat{\omega}^{3}=\hat{\omega}$,

i.e. we result either in a contradiction or in the trivial case $\hat{\omega} \equiv 0$. In principle, that should be expected since $\hat{\omega}$ is a pseudo-scalar operator as distinct from the other operators.

In the remainder of this section, we would like to return to analysis of the integral convolution (15.2). As was discussed in Sect. 15, we proved the validity of (15.2) in Sect. 7 and in Appendix F, where as the function $\tilde{\Omega}\left(\bar{\xi}^{\prime}, \xi\right)$ we had taken an expression from the matrix element for the Geyer operator $a_{0}^{2}$, Eqs. (12.3) and (12.4). However, in the extended statevector space $\mathfrak{A}_{G}$ instead of the operator $a_{0}^{2}$ we must use the operator $\hat{\omega}^{2} \equiv\left(a_{0}\right)^{2}$, whose matrix element by virtue of (17.7) has the form

$\left\langle\bar{\xi}^{\prime}\left|\hat{\omega}^{2}\right| \xi\right\rangle=\widetilde{\widetilde{\Omega}}\left(\bar{\xi}^{\prime}, \xi\right)\left\langle\bar{\xi}^{\prime} \mid \xi\right\rangle$,

where now

$\widetilde{\widetilde{\Omega}}\left(\bar{\xi}^{\prime}, \xi\right)=\frac{3}{4} \widetilde{\Omega}\left(\bar{\xi}^{\prime}, \xi\right)+\frac{1}{8}\left(\mathcal{C}_{2}\left(\bar{\xi}^{\prime}, \xi\right)-6\right)$. 
We define the function $\mathcal{C}_{2}\left(\bar{\xi}^{\prime}, \xi\right)$ as usually by the relation

$\left\langle\bar{\xi}^{\prime}\left|\widehat{C}_{2}\right| \xi\right\rangle=\mathcal{C}_{2}\left(\bar{\xi}^{\prime}, \xi\right)\left\langle\bar{\xi}^{\prime} \mid \xi\right\rangle$.

An explicit form of $\mathcal{C}_{2}(\bar{\xi}, \xi)$ is given by the expression (17.17). Instead of (15.2) we need to prove the validity of the relation

$\Omega\left(\bar{\xi}^{\prime}, \xi\right)=\Omega\left(\bar{\xi}^{\prime}, \xi\right) * \widetilde{\Omega}\left(\bar{\xi}^{\prime}, \xi\right)$.

For the first term on the right-hand side of (19.8) the relation (15.2) is still true and therefore instead of (19.9) we can write $\Omega\left(\bar{\xi}^{\prime}, \xi\right)=\frac{1}{2} \Omega\left(\bar{\xi}^{\prime}, \xi\right) *\left(\mathcal{C}_{2}\left(\bar{\xi}^{\prime}, \xi\right)-6\right)$

or

$\Omega\left(\bar{\xi}^{\prime}, \xi\right)=\frac{1}{8} \Omega\left(\bar{\xi}^{\prime}, \xi\right) * \mathcal{C}_{2}\left(\bar{\xi}^{\prime}, \xi\right)$.

However, a straightforward calculation of the convolution in (19.10) is too cumbersome, consequently we consider the proof of (19.10) within the operator formalism which is somewhat easier.

We exploit the fact that the right-hand side of (19.10) can be written as

$\frac{1}{8}\left\langle\bar{\xi}^{\prime}\left|a_{0} \widehat{C}_{2}\right| \xi\right\rangle\left\langle\bar{\xi}^{\prime} \mid \xi\right\rangle^{-1}$

In the matrix representation the following relation (see (H.7)) was proved:

$\omega B=2 \omega$.

In the operator representation it takes the form

$a_{0} \hat{\Lambda}=4 a_{0}$.

Further we use the representation of the Casimir operator $\hat{C}_{2}$ in terms of the operator $\hat{\Lambda}$, Eq. (17.10). Then by virtue of (19.13) the following chain of equalities is true:

$a_{0} \widehat{C}_{2}=-\frac{1}{2} a_{0} \hat{\Lambda}^{2}+4 a_{0} \hat{\Lambda}=\left[-\frac{1}{2} 4^{2}+4^{2}\right] a_{0}=8 a_{0}$.

The expression (19.11) in view of the last relation is really equals $\Omega\left(\bar{\xi}^{\prime}, \xi\right)$. However, we need clearly to show that the operator equality (19.13) indeed takes place.

We rewrite the equality (19.13) in an identical form

$\frac{1}{2}\left\{a_{0}, \hat{\Lambda}\right\}+\frac{1}{2}\left[a_{0}, \hat{\Lambda}\right]=4 a_{0}$.

Let us prove that the following relations are valid:

$\left\{a_{0}, \hat{\Lambda}\right\}=8 a_{0}, \quad\left[a_{0}, \hat{\Lambda}\right]=0$.

Here, we consider the proof of the first relation, the proof of the second one is given in Appendix I. Substituting an explicit form of the operators $a_{0}$ and $\hat{\Lambda}$ into the first relation in (19.14), we get

$$
\begin{gathered}
\left(\left\{\left\{L_{12}, M_{12}\right\},\left\{a_{1}^{+}, a_{1}^{-}\right\}\right\}+\left\{\left\{N_{12}, N_{21}\right\},\left\{a_{1}^{+}, a_{1}^{-}\right\}\right\}\right. \\
\left.-\quad\left\{\left\{N_{1}, N_{2}\right\},\left\{a_{1}^{+}, a_{1}^{-}\right\}\right\}\right)+(1 \rightleftarrows 2)
\end{gathered}
$$

$$
=8\left(\left\{L_{12}, M_{12}\right\}+\left\{N_{12}, N_{21}\right\}-\left\{N_{1}, N_{2}\right\}\right) .
$$

We will need two operator identities

$\{C,\{A, B\}\}=[A,[B, C]]+\{B,\{A, C\}\}$,

$[C,\{A, B\}]=\{B,[C, A]\}-\{A,[B, C]\}$.

We consider the first term on the left-hand side of (19.15). By using the identity (19.16), we obtain

$$
\begin{aligned}
& \left\{\left\{L_{12}, M_{12}\right\},\left\{a_{1}^{+}, a_{1}^{-}\right\}\right\} \\
& \quad=\left[L_{12},\left[M_{12},\left\{a_{1}^{+}, a_{1}^{-}\right\}\right]\right]+\left\{M_{12},\left\{\left\{a_{1}^{+}, a_{1}^{-}\right\}, L_{12}\right\}\right\} .
\end{aligned}
$$

Further we take advantage of the identity (19.17). The internal commutator in the first term in (19.18) is

$$
\begin{aligned}
{\left[M_{12},\left\{a_{1}^{+}, a_{1}^{-}\right\}\right] } & =\left\{a_{1}^{-},\left[M_{12}, a_{1}^{+}\right]\right\}-\left\{a_{1}^{+},\left[a_{1}^{-}, M_{12}\right]\right\} \\
& =-\left\{a_{1}^{-}, a_{2}^{-}\right\} .
\end{aligned}
$$

Here, we have used the commutation rules (6.15). Then the first term in (19.18) takes the form

$$
\begin{aligned}
-\left[L_{12},\left\{a_{1}^{-}, a_{2}^{-}\right\}\right] & =-\left\{a_{2}^{-},\left[L_{12}, a_{1}^{-}\right]\right\}+\left\{a_{1}^{-},\left[a_{2}^{-}, L_{12}\right]\right\} \\
& =\left\{a_{2}^{+}, a_{2}^{-}\right\}-\left\{a_{1}^{+}, a_{1}^{-}\right\},
\end{aligned}
$$

where we again have used the identity (19.17) and the rules (6.15).

Let us analyze now the second term on the right-hand side of (19.18). We have the following chain of equalities:

$$
\begin{aligned}
\left\{\left\{a_{1}^{+}, a_{1}^{-}\right\}, L_{12}\right\} & =\left[a_{1}^{+},\left[a_{1}^{-}, L_{12}\right]\right]+\left\{a_{1}^{-},\left\{L_{12}, a_{1}^{+}\right\}\right\} \\
& =\left[a_{1}^{+}, a_{2}^{+}\right]+\left\{a_{1}^{-},\left\{L_{12}, a_{1}^{+}\right\}\right\} \\
& =2 L_{12}-\frac{1}{2}\left\{a_{1}^{-},\left[a_{2}^{+},\left(a_{1}^{+}\right)^{2}\right]\right\} .
\end{aligned}
$$

Here, at the last step we have made transformation with the use of the definition of the generator $L_{12}$, Eq. (6.9),

$$
\left\{L_{12}, a_{1}^{+}\right\}=\frac{1}{2}\left\{\left[a_{1}^{+}, a_{2}^{+}\right], a_{1}^{+}\right\} \equiv-\frac{1}{2}\left[a_{2}^{+},\left(a_{1}^{+}\right)^{2}\right] .
$$

By this means the second term in (19.18) can be presented in the following form:

$$
\begin{aligned}
\left\{M_{12},\left\{\left\{a_{1}^{+}, a_{1}^{-}\right\}, L_{12}\right\}\right\}= & 2\left\{M_{12}, L_{12}\right\} \\
& -\frac{1}{2}\left\{M_{12},\left\{a_{2}^{-},\left[a_{2}^{+},\left(a_{1}^{+}\right)^{2}\right]\right\}\right\} .
\end{aligned}
$$

With the use of this expression and (19.19) the first contribution on the left-hand side of (19.15) is written as

$$
\begin{aligned}
& \left\{\left\{L_{12}, M_{12},\right\},\left\{a_{1}^{+}, a_{1}^{-}\right\}\right\} \\
& =\left(\left\{a_{2}^{+}, a_{2}^{-}\right\}-\left\{a_{1}^{+}, a_{1}^{-}\right\}\right)+2\left\{M_{12}, L_{12}\right\} \\
& \quad-\frac{1}{2}\left\{M_{12},\left\{a_{1}^{-},\left[a_{2}^{+},\left(a_{1}^{+}\right)^{2}\right]\right\}\right\} .
\end{aligned}
$$

Analysis of the second and the third contributions on the left-hand side of (19.15) is performed in accordance with the 
same scheme, because of this, here we write out only the final expressions:

for the second term

$$
\begin{aligned}
& \left\{\left\{N_{12}, N_{21}\right\},\left\{a_{1}^{+}, a_{1}^{-}\right\}\right\} \\
& =\left(-\left\{a_{2}^{+}, a_{2}^{-}\right\}+\left\{a_{1}^{+}, a_{1}^{-}\right\}\right)+2\left\{N_{21}, N_{12}\right\} \\
& \quad-\frac{1}{2}\left\{N_{21},\left\{a_{1}^{-},\left[a_{2}^{-},\left(a_{1}^{+}\right)^{2}\right]\right\}\right\}
\end{aligned}
$$

and for the third term

$$
\left\{\left\{N_{1}, N_{2}\right\},\left\{a_{1}^{+}, a_{1}^{-}\right\}\right\}=4\left\{N_{1}, N_{2}\right\} .
$$

It remains for us to analyze the last terms in (19.20) and (19.21). Let us consider the first of them. Making use of the operator identity (19.17) for the internal anticommutator we have

$$
\begin{aligned}
\left\{a_{1}^{-},\left[a_{2}^{+},\left(a_{1}^{+}\right)^{2}\right]\right\} & =\left\{\left(a_{1}^{+}\right)^{2},\left[a_{1}^{-}, a_{2}^{+}\right]\right\}+\left[a_{2}^{+},\left\{\left(a_{1}^{+}\right)^{2}, a_{1}^{-}\right\}\right] \\
& =-2\left\{\left(a_{1}^{+}\right)^{2}, N_{21}\right\}-4 L_{12} .
\end{aligned}
$$

Then this term takes the form

$$
\begin{aligned}
2\{ & \left.M_{12}, L_{12}\right\}+\left\{M_{12},\left\{\left(a_{1}^{+}\right)^{2}, N_{21}\right\}\right\} \\
= & 2\left\{M_{12}, L_{12}\right\}+\left[\left(a_{1}^{+}\right)^{2},\left[N_{21}, M_{12}\right]\right] \\
& +\left\{N_{21},\left\{M_{12},\left(a_{1}^{+}\right)^{2}\right\}\right\} .
\end{aligned}
$$

Here, we have used the identity (19.16). The second term on the right-hand side of the above expression vanishes by virtue of the algebra (6.11) and as a result, we obtain

$$
\begin{aligned}
- & \frac{1}{2}\left\{M_{12},\left\{a_{2}^{-},\left[a_{2}^{+},\left(a_{1}^{+}\right)^{2}\right]\right\}\right\} \\
& =2\left\{M_{12}, L_{12}\right\}+\left\{N_{21},\left\{M_{12},\left(a_{1}^{+}\right)^{2}\right\}\right\} .
\end{aligned}
$$

Completely similar analysis for the last term in (19.21) leads us to

$$
\begin{aligned}
- & \frac{1}{2}\left\{N_{21},\left\{a_{2}^{-},\left[a_{2}^{-},\left(a_{1}^{+}\right)^{2}\right]\right\}\right\} \\
& =2\left\{N_{21}, N_{12}\right\}-\left\{N_{21},\left\{\left(a_{1}^{+}\right)^{2}, M_{12}\right\}\right\}
\end{aligned}
$$

and thus, instead of (19.20) and (19.21), now we can write, correspondingly,

$$
\begin{aligned}
& \left\{\left\{L_{12}, M_{12}\right\},\left\{a_{1}^{+}, a_{1}^{-}\right\}\right\} \\
& =\left(\left\{a_{2}^{+}, a_{2}^{-}\right\}-\left\{a_{1}^{+}, a_{1}^{-}\right\}\right)+4\left\{M_{12}, L_{12}\right\} \\
& \quad+\left\{N_{21},\left\{M_{12},\left(a_{1}^{+}\right)^{2}\right\}\right\}, \\
& \left\{\left\{N_{12}, N_{21}\right\},\left\{a_{1}^{+}, a_{1}^{-}\right\}\right\} \\
& \quad=\left(\left\{a_{1}^{+}, a_{1}^{-}\right\}-\left\{a_{2}^{+}, a_{2}^{-}\right\}\right)+4\left\{N_{21}, N_{12}\right\} \\
& \quad-\left\{N_{21},\left\{M_{12},\left(a_{1}^{+}\right)^{2}\right\}\right\} .
\end{aligned}
$$

Putting the last expressions together and subtracting (19.22) from them, we obtain that the contribution of the first three terms on the left-hand side of (19.15) equals

$4\left(\left\{L_{12}, M_{12}\right\}+\left\{N_{21}, N_{12}\right\}-\left\{N_{1}, N_{2}\right\}\right)$.
The contribution with the replacement $(1 \rightleftarrows 2)$ gives us the remaining half of terms on the left-hand side of (19.15). In this way we really reproduce the first relation in (19.14). In fact we have proved even a more weak statement. Let us present the operator $\hat{\Lambda}$ as a sum

$\hat{\Lambda}=\hat{\Lambda}_{1}+\hat{\Lambda}_{2}$

where $\hat{\Lambda}_{1} \equiv\left\{a_{1}^{+}, a_{1}^{-}\right\}, \hat{\Lambda}_{2} \equiv\left\{a_{2}^{+}, a_{2}^{-}\right\}$. Then by virtue of the aforementioned we proved the validity of two independent relations

$\left\{a_{0}, \hat{\Lambda}_{1}\right\}=4 a_{0}, \quad\left\{a_{0}, \hat{\Lambda}_{2}\right\}=4 a_{0}$.

However, if we remember an existence of the second relation in (19.14), then in addition to (19.23) we have to verify the validity of the equalities

$\left[a_{0}, \hat{\Lambda}_{1}\right]=0, \quad\left[a_{0}, \hat{\Lambda}_{2}\right]=0$.

As we showed in Appendix I, the relations (19.24) do not hold separately, and they hold only in a sum.

The necessity of using the operator $\hat{\omega}^{2}$ instead of $a_{0}^{2}$ leads to a modification of some expressions with $a_{0}^{2}$, which were obtained earlier. In particular, it is concerned with the matrix elements $\left\langle\bar{\xi}^{\prime}\left|\left[a_{0}^{2}, a_{n}^{ \pm}\right]\right| \xi\right\rangle$ derived in Sect. 10. It is not difficult to show that now, instead of (10.21) and (10.22), we must use the equalities

$$
\begin{aligned}
& \left\langle\bar{\xi}^{\prime}\left|\left[\hat{\omega}^{2}, a_{n}^{+}\right]\right| \xi\right\rangle=-\left(\frac{\partial \widetilde{\widetilde{\Omega}}}{\partial \xi_{n}}\right)\left\langle\bar{\xi}^{\prime} \mid \xi\right\rangle, \\
& \left\langle\bar{\xi}^{\prime}\left|\left[\hat{\omega}^{2}, a_{n}^{-}\right]\right| \xi\right\rangle=-\left(\frac{\partial \widetilde{\widetilde{\Omega}}}{\partial \bar{\xi}_{n}^{\prime}}\right)\left\langle\bar{\xi}^{\prime} \mid \xi\right\rangle
\end{aligned}
$$

with the function $\widetilde{\widetilde{\Omega}}\left(\bar{\xi}^{\prime}, \xi\right)$ defined by the formula (19.8). Some other modifications of the formalism under consideration will be discussed below.

\section{Calculation of the commutators $\left[\hat{A}, a_{k}^{+}\right]$and

$$
\left[\hat{A},\left[a_{0}, a_{k}^{+}\right]\right]
$$

We determine the commutators of the operator $\hat{A}$ with the creation operator $a_{k}^{+}$and with the commutator $\left[a_{0}, a_{k}^{+}\right]$. The aim of these calculations is to obtain a more compact and visual representation for the matrix elements $\left\langle\bar{\xi}^{\prime}\left|\hat{A} a_{n}^{+}\right| \xi\right\rangle$ and $\left\langle\bar{\xi}^{\prime}\left|\hat{A}\left[a_{0}, a_{n}^{+}\right]\right| \xi\right\rangle$. We recall that the awkwardness of these matrix elements is caused by the necessity to move the operator $a_{k}^{+}$and the commutator $\left[a_{0}, a_{k}^{+}\right]$towards the left of the operator $\hat{A}$, Eqs. (10.2) and (11.9). For the calculation of the required commutators it is the most convenient for us to use the exponential representation of the operator $\hat{A}$

$\hat{A}=\alpha \mathrm{e}^{-i \frac{2 \pi}{3} a_{0}}$ 
We need the expression for the trilinear commutator (2.9), which for convenient reference, we give once more

$$
\left[a_{0},\left[a_{0}, a_{k}^{+}\right]\right]=a_{k}^{+} .
$$

First we consider the commutator $\left[\hat{A}, a_{k}^{+}\right]$. We rearrange the operator $\hat{A}$ to the right

$$
\begin{aligned}
& \alpha\left[\mathrm{e}^{-i \frac{2 \pi}{3} a_{0}}, a_{k}^{+}\right] \\
& =\alpha\left(\mathrm{e}^{-i \frac{2 \pi}{3} a_{0}} a_{k}^{+} \mathrm{e}^{i \frac{2 \pi}{3} a_{0}}-a_{k}^{+}\right) \mathrm{e}^{-i \frac{2 \pi}{3} a_{0}} \\
& =\left(a_{k}^{+}-\left(\frac{2 \pi i}{3}\right)\left[a_{0}, a_{k}^{+}\right]\right. \\
& \left.+\frac{1}{2 !}\left(\frac{2 \pi i}{3}\right)^{2}\left[a_{0},\left[a_{0}, a_{k}^{+}\right]\right]-\ldots-a_{k}^{+}\right) \hat{A} \\
& =\left(-\left\{\left(\frac{2 \pi i}{3}\right)+\frac{1}{3 !}\left(\frac{2 \pi i}{3}\right)^{3}+\cdots\right\}\left[a_{0}, a_{k}^{+}\right]\right. \\
& \left.+\left\{\frac{1}{2 !}\left(\frac{2 \pi i}{3}\right)^{2}+\frac{1}{4 !}\left(\frac{2 \pi i}{3}\right)^{4}+\cdots\right\} a_{k}^{+}\right) \hat{A} \\
& =\left\{-i \sin \frac{2 \pi i}{3}\left[a_{0}, a_{k}^{+}\right]+\cos \frac{2 \pi i}{3} a_{k}^{+}\right\} \hat{A} \\
& =\left\{\left(-\frac{i \sqrt{3}}{2}\right)\left[a_{0}, a_{k}^{+}\right]+\left(-\frac{3}{2}\right) a_{k}^{+}\right\} \hat{A} \text {. }
\end{aligned}
$$

Here, we have used the operator identity (5.3) and the commutation rule (20.2). The commutator $\left[\hat{A},\left[a_{0}, a_{k}^{+}\right]\right]$is calculated in the same way ${ }^{7}$ and as a result, we can write the desired commutators

$$
\begin{aligned}
& {\left[\hat{A}, a_{k}^{+}\right]=\left\{\left(-\frac{3}{2}\right) a_{k}^{+}+\left(-\frac{i \sqrt{3}}{2}\right)\left[a_{0}, a_{k}^{+}\right]\right\} \hat{A}, \quad} \\
& {\left[\hat{A},\left[a_{0}, a_{k}^{+}\right]\right]=\left\{\left(-\frac{i \sqrt{3}}{2}\right) a_{k}^{+}+\left(-\frac{3}{2}\right)\left[a_{0}, a_{k}^{+}\right]\right\} \hat{A} .}
\end{aligned}
$$

We analyze these expressions by calculating their matrix elements in the basis of parafermion coherent states. Let us consider the second one. For the left-hand side we make use of the relation ${ }^{8}(11.11)$

$$
\left[\hat{A},\left[a_{0}, a_{k}^{+}\right]\right]=-\beta a_{k}^{+}+2 \gamma a_{k}^{+} a_{0}+\gamma\left[a_{0}, a_{k}^{+}\right] .
$$

\footnotetext{
${ }^{7}$ It is easiest to calculate the double commutator $\left[\hat{A},\left[a_{0}, a_{k}^{+}\right]\right]$by an algebraic differentiation, i.e. by the commutation of the expression for $\left[\hat{A}, a_{k}^{+}\right]$with the operator $a_{0}$, in view of (20.2).

8 Recall that

$$
\beta=\left(\frac{i \sqrt{3}}{2}\right) \alpha, \quad \gamma=\left(-\frac{3}{2}\right) \alpha, \quad \alpha^{3}=\frac{1}{m} .
$$

The matrix elements of two terms on the right-hand side (20.3b) can be written as

$$
\begin{aligned}
& \left\langle\bar{\xi}^{\prime}\left|a_{k}^{+} \hat{A}\right| \xi\right\rangle=\bar{\xi}_{k}^{\prime} \mathcal{A}\left(\bar{\xi}^{\prime}, \xi\right)\left\langle\bar{\xi}^{\prime} \mid \xi\right\rangle, \\
& \left\langle\bar{\xi}^{\prime}\left|\left[a_{0}, a_{k}^{+}\right] \hat{A}\right| \xi\right\rangle=-\left(\frac{\partial \Omega\left(\bar{\xi}^{\prime}, \xi\right)}{\partial \xi_{k}}\right) * \mathcal{A}\left(\bar{\xi}^{\prime}, \xi\right)\left\langle\bar{\xi}^{\prime} \mid \xi\right\rangle .
\end{aligned}
$$

Here, we have used the definitions of the function $\mathcal{A}\left(\hat{\xi}^{\prime}, \xi\right)$, Eq. (10.23), of the derivative $\partial \Omega / \partial \xi_{k}$, Eq. (10.12), and of the star product (14.3). Based on (20.5) and (20.6) the matrix element of (20.3b) can be presented in the form

$$
\begin{aligned}
& (\alpha / \gamma)\left\{\bar{\xi}_{k}^{\prime}(-\beta+2 \gamma \Omega+(\beta / \alpha) \mathcal{A})-\gamma\left(\frac{\partial \Omega}{\partial \xi_{k}}\right)\right\} \\
& =-\left(\frac{\partial \Omega}{\partial \xi_{k}}\right) * \mathcal{A} .
\end{aligned}
$$

On the left and right-hand sides we canceled by the overlap function $\left\langle\bar{\xi}^{\prime} \mid \xi\right\rangle$.

Further we consider the relation (20.3a). Taking into account that

$$
\left\langle\bar{\xi}^{\prime}\left|\left[\hat{A}, a_{k}^{+}\right]\right| \xi\right\rangle=-\frac{\partial \mathcal{A}\left(\bar{\xi}^{\prime}, \xi\right)}{\partial \xi_{k}}\left\langle\bar{\xi}^{\prime} \mid \xi\right\rangle
$$

we can write the matrix element of the operator relation (20.3a) in the following form:

$(\alpha / \beta)\left\{(\gamma / \alpha) \bar{\xi}_{k}^{\prime} \mathcal{A}+\frac{\partial \mathcal{A}}{\partial \xi_{k}}\right\}=-\left(\frac{\partial \Omega}{\partial \xi_{k}}\right) * \mathcal{A}$.

Equating the left-hand sides of (20.7) and (20.8), we obtain a condition for consistency of the operator relations (20.3a) and (20.3b) in the enlarged state-vector space $\mathfrak{A}_{G}$ :

$$
\begin{aligned}
& \frac{1}{\gamma}\left\{\bar{\xi}_{k}^{\prime}(-\beta+2 \gamma \Omega+(\beta / \alpha) \mathcal{A})-\gamma\left(\frac{\partial \Omega}{\partial \xi_{k}}\right)\right\} \\
& \quad=\frac{1}{\beta}\left\{(\gamma / \alpha) \bar{\xi}_{k}^{\prime} \mathcal{A}+\frac{\partial \mathcal{A}}{\partial \xi_{k}}\right\} .
\end{aligned}
$$

This relation must be fulfilled identically. Let us verify this circumstance. The function $\mathcal{A}\left(\bar{\xi}^{\prime}, \xi\right)$ by virtue of the definitions (15.4) and (19.7) has the following form:

$\mathcal{A}\left(\bar{\xi}^{\prime}, \xi\right)=\alpha-\beta \Omega\left(\bar{\xi}^{\prime}, \xi\right)+\gamma \widetilde{\widetilde{\Omega}}\left(\bar{\xi}^{\prime}, \xi\right)$,

where, in turn, the function $\widetilde{\Omega}$ is given by the expression (19.8). Making use of the representation (9.18) for the function $\widetilde{\Omega}$, we have

$$
\frac{\partial \widetilde{\Omega}}{\partial \xi_{k}}=\bar{\xi}_{k}^{\prime}(2 \widetilde{\Omega}-1)
$$

Taking into account the last expression and (19.8), we obtain the derivative of the function $\mathcal{A}$ with respect to $\xi_{k}$ :

$$
\frac{\partial \mathcal{A}}{\partial \xi_{k}}=-\beta \frac{\partial \Omega}{\partial \xi_{k}}+\gamma \frac{3}{4} \bar{\xi}_{k}^{\prime}(2 \widetilde{\Omega}-1)+\gamma \frac{1}{8} \frac{\partial \mathcal{C}_{2}}{\partial \xi_{k}} .
$$


Substituting this derivative into (20.9), by using numerical values of the parameters $\beta$ and $\gamma$, Eq. (20.4), one can show that eventually the consistency condition (20.9) is reduced to the simple relation

$\frac{\partial \mathcal{C}_{2}\left(\bar{\xi}^{\prime}, \xi\right)}{\partial \xi_{k}}=2 \bar{\xi}_{k}^{\prime}\left(\mathcal{C}_{2}\left(\bar{\xi}^{\prime}, \xi\right)-7\right)$.

Finally, by using an explicit form of the function $\mathcal{C}_{2}\left(\bar{\xi}^{\prime}, \xi\right)$, Eq. (17.17), it is not difficult to verify that the consistency condition does not turn into identity. Let us define the reason of such a contradiction.

In deriving the formulas (20.3) and (20.5) we used the quantization rule (20.2). In a special case of parastatistics $p=2$ this rule is a consequence of two relations

$a_{0} a_{k}^{+} a_{0}=0, \quad\left(a_{0}\right)^{2} a_{k}^{+}+a_{k}^{+}\left(a_{0}\right)^{2}=a_{k}^{+}$,

which, in turn, is the operator representation of matrix relations (see Appendix A)

$\omega \beta_{\mu} \omega=0, \quad \omega^{2} \beta_{\mu}+\beta_{\mu} \omega^{2}=\beta_{\mu}$.

Let us define the matrix element in the basis of parafermion coherent states of the second operator relation in (20.13). For the left-hand side we have

$$
\begin{aligned}
\left\langle\bar{\xi}^{\prime}\left|\left\{\left(a_{0}\right)^{2}, a_{k}^{+}\right\}\right| \xi\right\rangle \equiv & \left\langle\bar{\xi}^{\prime}\left|2 a_{k}^{+}\left(a_{0}\right)^{2}+\left[\left(a_{0}\right)^{2}, a_{k}^{+}\right]\right| \xi\right\rangle \\
= & 2 \bar{\xi}_{k}^{\prime}\left\langle\bar{\xi}^{\prime}\left|\left(a_{0}\right)^{2}\right| \xi\right\rangle \\
& +\left\langle\bar{\xi}^{\prime}\left|\left[\left(a_{0}\right)^{2}, a_{k}^{+}\right]\right| \xi\right\rangle .
\end{aligned}
$$

At first we take as the operator $\left(a_{0}\right)^{2}$ the Geyer operator (2.17). Then making use of (10.19) and (10.21), we can put the desired matrix element after canceling by the overlap function into the form

$2 \bar{\xi}_{k}^{\prime} \widetilde{\Omega}-\frac{\partial \widetilde{\Omega}}{\partial \xi_{k}}=\bar{\xi}_{k}^{\prime}$,

and thus we result in (20.11), i.e. there is no contradiction. However, as we already know from the preceding consideration, instead of the Geyer operator $a_{0}^{2}$, we must use the Harish-Chandra operator $\hat{\omega}^{2} \equiv\left(a_{0}\right)^{2}$ and therefore, instead of (20.15), we have to write

$2 \bar{\xi}_{k}^{\prime} \widetilde{\Omega}-\frac{\partial \widetilde{\widetilde{\Omega}}}{\partial \xi_{k}}=\bar{\xi}_{k}^{\prime}$.

Substituting (19.8) into this equality, we result in the relation (20.12), which is contradictory. This circumstance provides a hint that the second matrix relation in (20.14), and as a consequence, its operator formulation (20.13) are not entirely correct.

In Appendix A we have given all basic formulas of the $\omega-\beta_{\mu}$ algebra. All of them except the formula (A.3) were proved in [6] without fixing the element of center (17.15). Let us discard this fixing in deriving the formula (A.3). We will need the relation (19.3) in which we come to the normalized matrix $\omega$ according to the rule $\omega \rightarrow 4 \omega$ :

$\omega^{2}=\frac{1}{4}[P(\theta)+Q(\theta) B]$.

Here we recall $P(\theta)=6(\theta-4)$ and $Q(\theta)=-2(\theta-4)$. By virtue of the fact that $\theta$ is element of the center of the DKP-algebra and from the equality $\left\{B, \beta_{\mu}\right\}=5 \beta_{\mu}$, instead of (A.3), we have

$$
\begin{aligned}
\left\{\omega^{2}, \beta_{\mu}\right\} & =\frac{1}{4}\left(2 P(\theta) \beta_{\mu}+Q(\theta)\left\{B, \beta_{\mu}\right\}\right) \\
& =\frac{1}{4}(2 \theta-8) \beta_{\mu} .
\end{aligned}
$$

To pass to the operator representation it is sufficient to perform the following replacements: $\omega \rightarrow \hat{\omega}\left(\equiv-a_{0}\right), \beta_{\mu} \rightarrow$ $a_{k}^{ \pm}, 2 \theta \rightarrow \widehat{C}_{2}^{\prime}$. Thus we finally arrive at the desired generalization of the second relation in (20.13)

$\left(a_{0}\right)^{2} a_{k}^{ \pm}+a_{k}^{ \pm}\left(a_{0}\right)^{2}=\frac{1}{4}\left(\widehat{C}_{2}^{\prime}-8\right) a_{k}^{ \pm}$.

In fixing the Casimir operator $\widehat{C}_{2}^{\prime}=12 \hat{I}$ we return to the original relation (20.13). With allowance made for this generalization, the double commutator (20.2) takes the form

$\left[a_{0},\left[a_{0}, a_{k}^{+}\right]\right]=\frac{1}{4}\left(\widehat{C}_{2}^{\prime}-8\right) a_{k}^{+}$,

and now, instead of (11.11), we obtain

$$
\begin{aligned}
{\left[\hat{A},\left[a_{0}, a_{k}^{+}\right]\right]=} & -\frac{1}{4} \beta\left(\widehat{C}_{2}^{\prime}-8\right) a_{k}^{+} \\
& +\frac{1}{4} \gamma\left\{a_{0}\left(\widehat{C}_{2}^{\prime}-8\right), a_{k}^{+}\right\} .
\end{aligned}
$$

Further, based on the representation (17.11) and on the property (19.13) we can put the last expression in the final form

$$
\begin{aligned}
& {\left[\hat{A},\left[a_{0}, a_{k}^{+}\right]\right]} \\
& \quad=-\frac{1}{4} \beta\left(\widehat{C}_{2}^{\prime}-8\right) a_{k}^{+}+2 \gamma a_{k}^{+} a_{0}+\gamma\left[a_{0}, a_{k}^{+}\right] .
\end{aligned}
$$

Comparing this expression with (20.5), we see that change occurred only in the first term on the right-hand side.

Further, we need to modify the relations (20.3), since now for their deriving we should make use of the commutation rules (20.16) instead of (20.2). It is not difficult to show that in this case, instead of (20.3), we get

$$
\begin{aligned}
{\left[\hat{A}, a_{k}^{+}\right]=} & \left\{\left(-\frac{3}{2}\right) a_{k}^{+}+\left(-\frac{i \sqrt{3}}{2}\right)\left[a_{0}, a_{k}^{+}\right]\right\} \hat{A} \\
& +\frac{1}{4} \gamma a_{k}^{+}\left(\widehat{C}_{2}^{\prime}-12\right), \\
{\left[\hat{A},\left[a_{0}, a_{k}^{+}\right]\right]=} & \left\{\left(-\frac{i \sqrt{3}}{2}\right) a_{k}^{+}+\left(-\frac{3}{2}\right)\left[a_{0}, a_{k}^{+}\right]\right\} \hat{A} \\
& -\frac{1}{4} \beta a_{k}^{+}\left(\widehat{C}_{2}^{\prime}-12\right) .
\end{aligned}
$$


Here, we see appearing additional terms in comparison with (20.3). At this stage on the left-hand side of (20.18b) we have to use the relation (20.17).

Let us consider now the matrix elements of the generalized operator relations (20.18). The change in the first term of the right-hand side of (20.17) leads to the following replacement on the left-hand side of (20.7):

$\bar{\xi}_{k}^{\prime} \beta \rightarrow \bar{\xi}_{k}^{\prime} \beta \frac{1}{4}\left(\mathcal{C}_{2}^{\prime}\left(\bar{\xi}^{\prime}, \xi\right)-8\right)$,

and additional terms in (20.18a) and (20.18b) result in appearance of additional terms on the left-hand side of the matrix elements (20.7) and (20.8), namely the expression

$-\bar{\xi}_{k}^{\prime} \beta \frac{1}{4}\left(\mathcal{C}_{2}^{\prime}\left(\bar{\xi}^{\prime}, \xi\right)-12\right)$,

should be added to the left-hand side of (20.7) and the expression

$\bar{\xi}_{k}^{\prime} \gamma \frac{1}{4}\left(\mathcal{C}_{2}^{\prime}\left(\bar{\xi}^{\prime}, \xi\right)-12\right)$

should be added to the left-hand side of (20.8). The consistency condition of thus obtained two matrix elements in view of the equality

$\mathcal{C}_{2}^{\prime}\left(\bar{\xi}^{\prime}, \xi\right)=\mathcal{C}_{2}\left(\bar{\xi}^{\prime}, \xi\right)+\Lambda\left(\bar{\xi}^{\prime}, \xi\right)$,

results us, instead of (20.12), in a completely different relation, namely

$\frac{\partial \mathcal{C}_{2}\left(\bar{\xi}^{\prime}, \xi\right)}{\partial \xi_{k}}=-2 \bar{\xi}_{k}^{\prime}\left(\Lambda\left(\bar{\xi}^{\prime}, \xi\right)-5\right)$.

By using an explicit form of the functions $\mathcal{C}_{2}\left(\bar{\xi}^{\prime}, \xi\right)$ and $\Lambda\left(\bar{\xi}^{\prime}, \xi\right)$, Eqs. (17.17) and (17.18), it is easy to verify that (20.19) turns into identity.

\section{Conclusion}

In this paper we have made the detailed analysis of the connection between the Duffin-Kemmer-Petiau algebra and an extended system of parafermion trilinear commutation relations for the creation and annihilation operators $a_{k}^{ \pm}$obeying para-Fermi statistics of order 2 and for an additional operator $a_{0}$. We have considered two representations of the operator $a_{0}$. The first of them is an "indirect" representation based on employing the resolvent $R$ of the Geyer operator $a_{0}^{2}$. The second is an "explicit" representation constructed from the generators of the group $S O(2 M)$. It was shown that the former in contrast to the latter leads to incorrect formulas determining the rules of action of the operator $a_{0}$ on the state vectors of the corresponding finite Fock space. We have suggested that the reason of such an inconsistency is that Geyer's expression for the operator $a_{0}^{2}$ in terms of the parafermion number operators is most probably not the square of the initial operator $a_{0}$. We recall that the latter appears as some additional abstract element of the algebra $\mathfrak{s o}(2 M+2)$.

As a secondary result we have obtained a simple elegant representation for the operator $a_{0}^{2}$ in terms of the parafermion parity operator $(-1)^{n}$, where $n$ is the parafermion number operator. This representation in particular enabled us to obtain the expression for some matrix elements in the basis of parafermion coherent states by a simple way in contrast to an approach based on the Geyer representation, Eq. (2.17). Besides we found an intriguing connection between the $a_{0}^{2}$ operator and the so-called $C P T$ operator $\widehat{\eta}_{5}$, Eq. (8.11).

An important element of the developed formalism is the notion of a star product of para-Grassmann-valued functions, which enabled to put the matrix elements into the elegant and compact form. Two different techniques of the calculation of the star product were considered. This made it possible to verify independently the results of the calculations.

Another important element of our formalism is the notion of an extended Fock space $\mathfrak{A}_{G}$. In fact, this notion is a key element of our approach. We have come to recognize that we must work within the framework of Ohnuki and Kamefuchi's generalized state-vector space $\mathfrak{A}_{G}$ rather than in the context of the usual state-vector space $\mathfrak{A}$ that allowed us finally to resolve all the contradictions involved. As a particular consequence, a number of the trilinear matrix and operator relations obtained earlier in the papers by Harish-Chandra [6] and by Geyer [22] have been refined. These improved relations include such an important objects as the element $\theta$ of the center of the Duffin-Kemmer-Petiau algebra and the quadratic Casimir operator $\hat{C}_{2}^{\prime}$ of the Lie group $S O(2 M+1)$.

Data Availability Statement This manuscript has no associated data or the data will not be deposited. [Authors' comment: This is a theoretical article and no experimental data has been listed.]

Open Access This article is licensed under a Creative Commons Attribution 4.0 International License, which permits use, sharing, adaptation, distribution and reproduction in any medium or format, as long as you give appropriate credit to the original author(s) and the source, provide a link to the Creative Commons licence, and indicate if changes were made. The images or other third party material in this article are included in the article's Creative Commons licence, unless indicated otherwise in a credit line to the material. If material is not included in the article's Creative Commons licence and your intended use is not permitted by statutory regulation or exceeds the permitted use, you will need to obtain permission directly from the copyright holder. To view a copy of this licence, visit http://creativecomm ons.org/licenses/by/4.0/.

Funded by $\mathrm{SCOAP}^{3}$.

\section{Appendix A: The $\omega-\beta_{\mu}$ matrix algebra}

In this Appendix we give some necessary formulas of the $\omega$ $-\beta_{\mu}$ matrix algebra, which are used in the article text. This algebra characterizes the matrices for the spin-1 case (for 
the 10-row representation in the four-dimensional Euclidean space-time). Details of the proof of these formulas and also their generalization to higher dimensions can be found in the papers by Harish-Chandra [6,47] and Fujiwara [7]. In view of the definition of the $\omega$ matrix, Eq. (3.2), and the trilinear relation for the $\beta$-matrices, Eq. (3.1), in arbitrary evendimensional $D=2 M$ Euclidean space-time we have

$\omega^{3}=\omega$,

$\omega \beta_{\mu} \omega=0$,

$\omega^{2} \beta_{\mu}+\beta_{\mu} \omega^{2}=\beta_{\mu}$,

$\beta_{\mu} \beta_{\nu} \omega+\omega \beta_{\nu} \beta_{\mu}=\omega \delta_{\mu \nu}$,

$\beta_{\mu} \omega \beta_{\nu}+\beta_{\nu} \omega \beta_{\mu}=0$.

If one defines the matrix $B \equiv \beta_{\mu} \beta_{\mu}$, then the following relation is valid:

$\omega^{2}=M+1-B$.

\section{Appendix B: Para-Grassmann numbers}

In this Appendix we list the most important commutation relations between the para-Grassmann numbers and the paraFermi operators $a_{i}^{ \pm}$. We follow the definition of a paraGrassmann algebra suggested by Omote and Kamefuchi [25], namely a set of independent numbers $\xi_{1}, \xi_{2}, \ldots, \xi_{M}$ are said to form a para-Grassmann algebra of order $p$ when these numbers satisfy the following relations:

$$
\begin{aligned}
& {\left[\xi_{i},\left[\xi_{j}, \xi_{k}\right]\right]=0,} \\
& \left\{\xi_{i_{1}}, \xi_{i_{2}}, \ldots, \xi_{i_{m}}\right\}=0 \text { for } m \geq p+1,
\end{aligned}
$$

where $i^{\prime} s, j, k=1,2, \ldots, M$ and by the symbol $\left\{\xi_{i_{1}}, \xi_{i_{2}}\right.$, $\ldots, \xi_{i_{m}}$ \} one means a product of $m \xi$-numbers completely symmetrized with respect to the indices $i_{1}, i_{2}, \ldots, i_{m}$. For the special case $p=2$ these relations are reduced to

$\xi_{i} \xi_{j} \xi_{k}+\xi_{k} \xi_{j} \xi_{i}=0$.

Further, let us write out the rules of commutation between the para-Grassmann numbers and the creation and annihilation para-Fermi operators $a_{i}^{ \pm}$:

$\left[a_{i}^{ \pm},\left[a_{j}^{\mp}, \xi_{k}\right]\right]=2 \delta_{i j} \xi_{k}$,

$\left[a_{i}^{ \pm},\left[a_{j}^{ \pm}, \xi_{k}\right]\right]=0$,

$\left[\xi_{i},\left[\xi_{j}, a_{k}^{ \pm}\right]\right]=0$.

These relations hold for parastatistics of an arbitrary order $p$. For the case $p=2$ the commutation rules (B.3) turn into identity on the strength of the following relations:

$a_{i}^{ \pm} a_{j}^{\mp} \xi_{k}+\xi_{k} a_{j}^{\mp} a_{i}^{ \pm}=2 \delta_{i j} \xi_{k}$,

$a_{i}^{ \pm} \xi_{k} a_{j}^{\mp}+a_{j}^{\mp} \xi_{k} a_{i}^{ \pm}=0$.

By direct calculations, one can verify a validity of these equalities using Green's decomposition [46]

$$
a_{i}^{ \pm}=\sum_{\alpha=1}^{2} a_{i}^{ \pm(\alpha)}, \quad \xi_{i}=\sum_{\alpha=1}^{2} \xi_{i}^{(\alpha)}
$$

and the bilinear commutation relations for the Green components $[26,46]$

$$
\begin{aligned}
& \left\{a_{i}^{ \pm(\alpha)}, a_{j}^{\mp(\alpha)}\right\}=\delta_{i j},\left\{a_{i}^{ \pm(\alpha)}, a_{j}^{ \pm(\alpha)}\right\}=0, \alpha=1,2, \\
& {\left[a_{k}^{ \pm(\alpha)}, a_{l}^{ \pm(\beta)}\right]=0, \quad\left[a_{k}^{ \pm(\alpha)}, a_{l}^{\mp(\beta)}\right]=0, \alpha \neq \beta,} \\
& \left\{a_{k}^{ \pm(\alpha)}, \xi_{l}^{(\alpha)}\right\}=0, \quad\left[a_{k}^{ \pm(\alpha)}, \xi_{l}^{(\beta)}\right]=0, \\
& \left\{\xi_{k}^{(\alpha)}, \xi_{l}^{(\alpha)}\right\}=0, \quad\left[\xi_{k}^{(\alpha)}, \xi_{l}^{(\beta)}\right]=0 .
\end{aligned}
$$

For the commutation rules (B.4) and (B.5) we can write out similar relations for the particular case $p=2$, correspondingly,

$$
\begin{aligned}
& a_{i}^{ \pm} a_{j}^{ \pm} \xi_{k}+\xi_{k} a_{j}^{ \pm} a_{i}^{ \pm}=0, \\
& a_{i}^{ \pm} \xi_{k} a_{j}^{ \pm}+a_{j}^{ \pm} \xi_{k} a_{i}^{ \pm}=0
\end{aligned}
$$

and

$$
\begin{aligned}
& \xi_{i} \xi_{j} a_{k}^{ \pm}+a_{k}^{ \pm} \xi_{j} \xi_{i}=0, \\
& \xi_{i} a_{k}^{ \pm} \xi_{j}+\xi_{j} a_{k}^{ \pm} \xi_{i}=0 .
\end{aligned}
$$

\section{Appendix C: Differentiation of para-Grassmann-valued functions}

Here, we present the formulas of differentiation with respect to a para-Grassmann number $\xi$. The required formulas for the left derivative are $[13,26]$

$$
\begin{aligned}
& \frac{\partial([\xi, \zeta] g(\xi))}{\partial \xi}=\left(\frac{\partial[\xi, \zeta]}{\partial \xi}\right) g(\xi)+[\xi, \zeta] \frac{\partial g(\xi)}{\partial \xi}, \\
& \frac{\partial}{\partial \xi}[\xi, \zeta]=2 \zeta \\
& \frac{\partial}{\partial \xi} \xi^{n}=n(p+1-n) \xi^{n-1} .
\end{aligned}
$$

In particular, from the last formula for $n=1$ and $p=2$ it follows that

$$
\frac{\partial}{\partial \xi} \xi=2 \text {. }
$$


Appendix D: Integration of para-Grassmann-valued functions

The general formulas of integration with respect to a single para-Grassmann variable $\mu$ of arbitrary order $p$ are given in [26]. Here, we write out only the necessary formulas for the special case $p=2$. The integrals different from zero have the form:

$$
\begin{aligned}
& \int \mu^{2} d^{2} \mu=2 i^{2}, \\
& \int[\mu, \xi] \mu d^{2} \mu=-2 i^{2} \xi, \\
& \int[\mu, \xi]\left[\mu, \xi^{\prime}\right] d^{2} \mu=2 i^{2}\left\{\xi, \xi^{\prime}\right\}, \\
& \int[\mu, \xi]\left\{\mu, \xi^{\prime}\right\} d^{2} \mu=2 i^{2}\left[\xi, \xi^{\prime}\right],
\end{aligned}
$$

where $\xi$ and $\xi^{\prime}$ are arbitrary para-Grassmann numbers of the same order as $\mu$. The integrals containing zeroth and first powers of $\mu$ vanish by the definition. Let us write out also the expression for the $\delta$-function

$\delta\left(\xi-\xi^{\prime}\right) \equiv \frac{1}{2 i^{2}}\left(\xi-\xi^{\prime}\right)^{2}=\int \mathrm{e}^{-\frac{1}{2}\left[\xi-\xi^{\prime}, \mu\right]} d^{2} \mu$.

\section{Appendix E: Algebra of the generators $L_{k l}, M_{k l}$ and $N_{k l}$}

In this Appendix we present a list of the commutation relations for the generators

$$
\begin{aligned}
L_{k l} & =\frac{1}{2}\left[a_{k}^{+}, a_{l}^{+}\right], \quad M_{k l}=\frac{1}{2}\left[a_{k}^{-}, a_{l}^{-}\right], \\
N_{k l} & =\frac{1}{2}\left[a_{k}^{+}, a_{l}^{-}\right],
\end{aligned}
$$

as they were defined in the paper by Kamefuchi and Takahashi [21]. Here, the indices $k$ and $l$ run values $1,2, \ldots, M$. These generators possess evident properties

$$
L_{k l}=-L_{l k}, \quad M_{k l}=-M_{l k}, \quad N_{k l}^{\dagger}=N_{l k}, \quad L_{k l}^{\dagger}=M_{l k}
$$

and satisfy the following commutation relations:

$$
\begin{aligned}
& {\left[N_{k l}, N_{m n}\right]=\delta_{l m} N_{k n}-\delta_{k n} N_{m l},} \\
& {\left[L_{k l}, N_{m n}\right]=\delta_{l n} L_{m k}-\delta_{k n} L_{m l},} \\
& {\left[L_{k l}, L_{m n}\right]=0, \quad\left[M_{k l}, N_{m n}\right]=\delta_{k m} M_{n l}-\delta_{l m} M_{n k},} \\
& {\left[M_{k l}, M_{m n}\right]=0,} \\
& {\left[L_{k l}, M_{m n}\right]=-\delta_{k m} N_{l n}+\delta_{k n} N_{l m}-\delta_{l n} N_{k m}+\delta_{l m} N_{k n} .}
\end{aligned}
$$

The commutation relations involving the operators $a_{k}^{ \pm}$have the form

$$
\begin{aligned}
& {\left[a_{k}^{-}, L_{l m}\right]=\delta_{k l} a_{m}^{+}-\delta_{k m} a_{l}^{+}, \quad\left[a_{k}^{-}, M_{l m}\right]=0,} \\
& {\left[a_{k}^{-}, N_{l m}\right]=\delta_{k l} a_{m}^{-},} \\
& {\left[a_{k}^{+}, M_{l m}\right]=\delta_{k l} a_{m}^{-}-\delta_{k m} a_{l}^{-},} \\
& {\left[a_{k}^{+}, L_{l m}\right]=0, \quad\left[a_{k}^{+}, N_{l m}\right]=-\delta_{k m} a_{l}^{+} .}
\end{aligned}
$$

\section{Appendix F: Proof of the relation (7.11)}

Our first step is to verify the validity of the relation

$$
(-1)^{n}|\xi\rangle=|-\xi\rangle \text {. }
$$

We have used this expression in Sect. 9 without proof. Let us present the left-hand side of (F.1) in the following form:

$$
\begin{aligned}
(-1)^{n}|\xi\rangle & =\mathrm{e}^{i \pi n} \mathrm{e}^{-\frac{1}{2} \sum_{l}\left[\xi_{l}, a_{l}^{+}\right]}|0\rangle \\
& \equiv\left(\mathrm{e}^{i \pi n} \mathrm{e}^{-\frac{1}{2} \sum_{l}\left[\xi_{l}, a_{l}^{+}\right]} \mathrm{e}^{-i \pi n}\right) \mathrm{e}^{i \pi n}|0\rangle \\
& =\exp \left[\left(-\frac{1}{2}\right) \mathrm{e}^{i \pi n} \sum_{l}\left[\xi_{l}, a_{l}^{+}\right] \mathrm{e}^{-i \pi n}\right]|0\rangle .
\end{aligned}
$$

Here, we have taken into account the operator identity

$\mathrm{e}^{X} \mathrm{e}^{Y} \mathrm{e}^{-X}=\exp \left(\mathrm{e}^{X} Y \mathrm{e}^{-X}\right)$.

Further, for the argument of the exponential in (F.2) we use the identity (5.3). Taking the commutation relations (B.3) and (B.4) into account, we have

$\sum_{l}\left[n,\left[\xi_{l}, a_{l}^{+}\right]\right]=\sum_{l}\left[\xi_{l}, a_{l}^{+}\right]$

and therefore

$$
\begin{aligned}
\mathrm{e}^{i \pi n} \sum_{l}\left[\xi_{l}, a_{l}^{+}\right] \mathrm{e}^{-i \pi n} \\
=\sum_{l}\left[\xi_{l}, a_{l}^{+}\right]\left(1+i \pi+\frac{1}{2 !}(i \pi)^{2}+\cdots\right) \\
=\sum_{l}\left[\xi_{l}, a_{l}^{+}\right] \mathrm{e}^{i \pi}=-\sum_{l}\left[\xi_{l}, a_{l}^{+}\right] .
\end{aligned}
$$

By this means from (F.2) it follows that

$$
(-1)^{n}|\xi\rangle=\mathrm{e}^{\frac{1}{2} \sum_{l}\left[\xi_{l}, a_{l}^{+}\right]}|0\rangle \equiv|-\xi\rangle .
$$

The matrix element of the expression (9.20) in the basis of the parafermion coherent states with allowance made for (F.2) takes the form

$$
\left\langle\bar{\xi}^{\prime}\left|a_{0}\right| \xi\right\rangle=\left\langle-\bar{\xi}^{\prime}\left|a_{0}\right| \xi\right\rangle \text {. }
$$

We rewrite the equality in the notations (10.9), (10.10)

$\Omega\left(\bar{\xi}^{\prime}, \xi\right)\left\langle\bar{\xi}^{\prime} \mid \xi\right\rangle=\Omega\left(-\bar{\xi}^{\prime}, \xi\right)\left\langle-\bar{\xi}^{\prime} \mid \xi\right\rangle$ 
or

$\Omega\left(\bar{\xi}^{\prime}, \xi\right)=\Omega\left(-\bar{\xi}^{\prime}, \xi\right) \exp \left(-\sum_{l}\left[\bar{\xi}_{l}^{\prime}, \xi_{l}\right]\right)$.

The last expression can be considered as a rule of changing a sign of the para-Grassmann variables $\xi_{1}$ and $\xi_{2}$ (or $\bar{\xi}_{1}^{\prime}$ and $\left.\bar{\xi}_{2}^{\prime}\right)$ of the function $\Omega$. Let us represent this function as a sum of the terms quadratic and linear in the commutators

$\Omega\left(\bar{\xi}^{\prime}, \xi\right)=\Delta \Omega\left(\bar{\xi}^{\prime}, \xi\right)-(x+y-1)$,

where

$$
\begin{aligned}
\Delta \Omega\left(\bar{\xi}^{\prime}, \xi\right)=- & \frac{1}{2}\left\{\left(\frac{1}{2}\left[\bar{\xi}_{1}^{\prime}, \bar{\xi}_{2}^{\prime}\right]\right)\left(\frac{1}{2}\left[\xi_{1}, \xi_{2}\right]\right)\right. \\
+ & \left.\left(\frac{1}{2}\left[\bar{\xi}_{1}^{\prime}, \xi_{2}\right]\right)\left(\frac{1}{2}\left[\bar{\xi}_{2}^{\prime}, \xi_{1}\right]\right)-x y\right\} .
\end{aligned}
$$

The notations $x$ and $y$ were introduced by us in Sect. 5, Eq. (5.7). Then the expression (F.3) can be written as

$$
\begin{aligned}
& {\left[\Delta \Omega\left(\bar{\xi}^{\prime}, \xi\right)-(x+y-1)\right] \mathrm{e}^{x+y}} \\
& \quad=\left[\Delta \Omega\left(\bar{\xi}^{\prime}, \xi\right)-(x+y-1)\right] \mathrm{e}^{-(x+y)}
\end{aligned}
$$

or collecting similar terms it takes the form

$\left.\left[\Delta \Omega\left(\bar{\xi}^{\prime}, \xi\right)+1\right)\right] \tanh (x+y)=x+y$.

In view of the algebra (5.8), further we obtain

$$
\begin{aligned}
\tanh (x+y) & =(x+y)-\frac{1}{3}(x+y)^{3} \\
& =(x+y)-\left(x^{2} y+x y^{2}\right) .
\end{aligned}
$$

Taking into account the expansion and the explicit form of the function $\Delta \Omega$, Eq. (F.4), instead of (F.5) we obtain

$$
\begin{aligned}
& \frac{1}{8}\left(\left[\bar{\xi}_{1}^{\prime}, \bar{\xi}_{2}^{\prime}\right]\left[\xi_{1}, \xi_{2}\right]+\left[\bar{\xi}_{1}^{\prime}, \xi_{2}\right]\left[\bar{\xi}_{2}^{\prime}, \xi_{1}\right]\right) \\
& \quad \times\left(\left[\bar{\xi}_{1}^{\prime}, \xi_{1}\right]+\left[\bar{\xi}_{2}^{\prime}, \xi_{2}\right]\right) \\
& \quad+\left(x^{2} y+x y^{2}\right)=0 .
\end{aligned}
$$

The terms linear in $x$ and $y$ were canceled. In further analysis of the expression (F.6) for the para-Grassmann numbers we have to use, instead of the general relations (B.1), the particular relation (B.2) valid only for para-Grassmann numbers of order $p=2$.

At first we deal with the expression

$$
\begin{aligned}
{\left[\bar{\xi}_{1}^{\prime}, \xi_{1}\right]^{2} } & =\left(\bar{\xi}_{1}^{\prime} \xi_{1}-\xi_{1} \bar{\xi}_{1}^{\prime}\right)\left(\bar{\xi}_{1}^{\prime} \xi_{1}-\xi_{1} \bar{\xi}_{1}^{\prime}\right) \\
& =-\bar{\xi}_{1}^{\prime}\left(\xi_{1}\right)^{2} \bar{\xi}_{1}^{\prime}-\xi_{1}\left(\bar{\xi}_{1}^{\prime}\right)^{2} \xi_{1} \\
& =2\left(\bar{\xi}_{1}^{\prime}\right)^{2}\left(\xi_{1}\right)^{2},
\end{aligned}
$$

i.e. for $p=2$ we get

$\left(\bar{\xi}_{1}^{\prime}\right)^{2}\left(\xi_{1}\right)^{2}=\frac{1}{2}\left[\bar{\xi}_{1}^{\prime}, \xi_{1}\right]^{2}=2 x^{2}$ and similarly

$\left(\bar{\xi}_{2}^{\prime}\right)^{2}\left(\xi_{2}\right)^{2}=\frac{1}{2}\left[\bar{\xi}_{2}^{\prime}, \xi_{2}\right]^{2}=2 y^{2}$

Let us consider the first contribution in the product on the left-hand side of (F.6)

$$
\begin{aligned}
& {\left[\bar{\xi}_{1}^{\prime}, \bar{\xi}_{2}^{\prime}\right]\left[\bar{\xi}_{1}^{\prime}, \xi_{1}\right]\left[\xi_{1}, \xi_{2}\right]} \\
& \quad=\left(-\bar{\xi}_{1}^{\prime} \bar{\xi}_{2}^{\prime} \xi_{1} \bar{\xi}_{1}^{\prime}-\bar{\xi}_{2}^{\prime} \bar{\xi}_{1}^{\prime} \bar{\xi}_{1}^{\prime} \xi_{1}\right)\left[\xi_{1}, \xi_{2}\right] \\
& =\bar{\xi}_{1}^{\prime} \bar{\xi}_{2}^{\prime} \xi_{1} \bar{\xi}_{1}^{\prime} \xi_{2} \xi_{1}-\bar{\xi}_{2}^{\prime} \bar{\xi}_{1}^{\prime} \bar{\xi}_{1}^{\prime} \xi_{1} \xi_{1} \xi_{2} \\
& =\xi_{2} \bar{\xi}_{2}^{\prime}\left(\xi_{1}\right)^{2}\left(\bar{\xi}_{1}^{\prime}\right)^{2}-\bar{\xi}_{2}^{\prime} \xi_{2}\left(\bar{\xi}_{1}^{\prime}\right)^{2}\left(\xi_{1}\right)^{2} \\
& =-\left[\bar{\xi}_{2}^{\prime}, \xi_{2}\right]\left(\bar{\xi}_{1}^{\prime}\right)^{2}\left(\xi_{1}\right)^{2} \\
& =-\frac{1}{2}\left[\bar{\xi}_{2}^{\prime}, \xi_{2}\right]\left[\bar{\xi}_{1}^{\prime}, \xi_{1}\right]^{2} \\
& =-4 y x^{2} .
\end{aligned}
$$

At the last step we have used relation (F.7). The remaining three contributions in a product in (F.6) are analyzed in a similar manner and as a result we can write

$$
\begin{aligned}
& {\left[\bar{\xi}_{1}^{\prime}, \bar{\xi}_{2}^{\prime}\right]\left[\bar{\xi}_{2}^{\prime}, \xi_{2}\right]\left[\xi_{1}, \xi_{2}\right]=-4 x y^{2},} \\
& {\left[\bar{\xi}_{2}^{\prime}, \xi_{1}\right]\left[\bar{\xi}_{1}^{\prime}, \xi_{2}\right]\left[\bar{\xi}_{1}^{\prime}, \xi_{1}\right]=-4 y x^{2},} \\
& {\left[\bar{\xi}_{2}^{\prime}, \xi_{1}\right]\left[\bar{\xi}_{1}^{\prime}, \xi_{2}\right]\left[\bar{\xi}_{2}^{\prime}, \xi_{2}\right]=-4 x y^{2},} \\
& {\left[\bar{\xi}_{1}^{\prime}, \bar{\xi}_{2}^{\prime}\right]\left[\xi_{1}, \xi_{2}\right]\left[\bar{\xi}_{1}^{\prime}, \xi_{1}\right]=-4 y x^{2} .}
\end{aligned}
$$

Substituting the obtained expressions into (F.6), we see that the latter turns into identity.

\section{Appendix G: A proof of the relations (2.7) and (2.8)}

Here, we show that the commutation rules (2.7) and (2.8) (and their consequences (2.10) and (2.11)) turn into identities after substitution of the operator $a_{0}$ in the representation (6.17). Let us consider at first the relation (2.8) and for the sake of concreteness we take

$\left[\left[a_{0}, a_{n}^{+}\right], a_{m}^{+}\right]=0$.

For the commutator $\left[a_{0}, a_{n}^{+}\right]$we use the first representation given in Sect. 10, namely the expressions (10.3) and (10.5). Then, we can write the double commutator in (G.1) in the following form:

$$
\begin{aligned}
& {\left[\left[a_{0}, a_{n}^{+}\right], a_{m}^{+}\right]} \\
& \quad=-\frac{1}{4}\left(\delta_{n 2}\left[\left\{L_{12}, a_{1}^{-}\right\}, a_{m}^{+}\right]-\delta_{n 1}\left[\left\{L_{12}, a_{2}^{-}\right\}, a_{m}^{+}\right]\right. \\
& \quad+\delta_{n 2}\left[\left\{N_{21}, a_{1}^{+}\right\}, a_{m}^{+}\right]+\delta_{n 1}\left[\left\{N_{12}, a_{2}^{+}\right\}, a_{m}^{+}\right] \\
& \left.\quad-\delta_{n 2}\left[\left\{N_{1}, a_{2}^{+}\right\}, a_{m}^{+}\right]-\delta_{n 1}\left[\left\{N_{2}, a_{1}^{+}\right\}, a_{m}^{+}\right]\right) .
\end{aligned}
$$

By using the identity (10.4), the definitions (6.9) and the relations (6.15), for each of the terms on the right-hand side 
of (G.2), we get

$$
\begin{aligned}
{\left[\left\{L_{12}, a_{1}^{-}\right\}, a_{m}^{+}\right] } & =-2\left\{L_{12}, N_{m 1}\right\} \\
& \equiv-2 \delta_{m 1}\left\{L_{12}, N_{1}\right\}-2 \delta_{m 2}\left\{L_{12}, N_{21}\right\}, \\
{\left[\left\{L_{12}, a_{2}^{-}\right\}, a_{m}^{+}\right] } & =-2\left\{L_{12}, N_{m 2}\right\} \\
& \equiv-2 \delta_{m 2}\left\{L_{12}, N_{2}\right\}-2 \delta_{m 1}\left\{L_{12}, N_{12}\right\}, \\
{\left[\left\{N_{21}, a_{1}^{+}\right\}, a_{m}^{+}\right] } & =2 \delta_{m 2}\left\{N_{21}, L_{12}\right\}+\delta_{m 1}\left\{a_{1}^{+}, a_{2}^{+}\right\}, \\
{\left[\left\{N_{12}, a_{2}^{+}\right\}, a_{m}^{+}\right] } & =-2 \delta_{m 1}\left\{N_{12}, L_{12}\right\}+\delta_{m 2}\left\{a_{2}^{+}, a_{1}^{+}\right\}, \\
{\left[\left\{N_{1}, a_{2}^{+}\right\}, a_{m}^{+}\right] } & =-2 \delta_{m 1}\left\{N_{1}, L_{12}\right\}+\delta_{m 1}\left\{a_{2}^{+}, a_{1}^{+}\right\}, \\
{\left[\left\{N_{2}, a_{1}^{+}\right\}, a_{m}^{+}\right] } & =2 \delta_{m 2}\left\{N_{2}, L_{12}\right\}+\delta_{m 2}\left\{a_{1}^{+}, a_{2}^{+}\right\} .
\end{aligned}
$$

Substituting these expressions into (G.2) and collecting similar terms with respect to Kronecker deltas, we derive

$$
\begin{aligned}
& {\left[\left[a_{0}, a_{n}^{+}\right], a_{m}^{+}\right] } \\
&=- \frac{1}{2}\left\{\delta_{n 2} \delta_{m 1}\left(-\left\{L_{12}, N_{1}\right\}+\left\{N_{1}, L_{12}\right\}\right)\right. \\
&+\delta_{n 2} \delta_{m 2}\left(-\left\{L_{12}, N_{21}\right\}+\left\{N_{21}, L_{12}\right\}\right) \\
&+\delta_{n 1} \delta_{m 1}\left(\left\{L_{12}, N_{12}\right\}-\left\{N_{12}, L_{12}\right\}\right) \\
&\left.+\delta_{n 1} \delta_{m 2}\left(\left\{L_{12}, N_{2}\right\}-\left\{N_{2}, L_{12}\right\}\right)\right\} \\
&-\frac{1}{4}\left\{\delta_{n 2} \delta_{m 1}\left(\left\{a_{1}^{+}, a_{2}^{+}\right\}-\left\{a_{2}^{+}, a_{1}^{+}\right\}\right)\right. \\
&\left.+\delta_{n 1} \delta_{m 2}\left(\left\{a_{2}^{+}, a_{1}^{+}\right\}-\left\{a_{1}^{+}, a_{2}^{+}\right\}\right)\right\} .
\end{aligned}
$$

Here we see that the right-hand side vanishes identically and thus the relation (G.1) seems to be true.

Let us consider now the relation (2.7) and to be specific, its particular case

$\left[\left[a_{0}, a_{n}^{+}\right], a_{m}^{-}\right]=2 \delta_{k m} a_{0}$.

Now for the left-hand side, instead of (G.2) and (G.3), we have

$$
\begin{aligned}
& {\left[\left[a_{0}, a_{n}^{+}\right], a_{m}^{-}\right]} \\
& =-\frac{1}{4}\left(\delta_{n 2}\left[\left\{L_{12}, a_{1}^{-}\right\}, a_{m}^{-}\right]-\delta_{n 1}\left[\left\{L_{12}, a_{2}^{-}\right\}, a_{m}^{-}\right]\right. \\
& \quad+\delta_{n 2}\left[\left\{N_{21}, a_{1}^{+}\right\}, a_{m}^{-}\right]+\delta_{n 1}\left[\left\{N_{12}, a_{2}^{+}\right\}, a_{m}^{-}\right] \\
& \left.\quad-\delta_{n 2}\left[\left\{N_{1}, a_{2}^{+}\right\}, a_{m}^{-}\right]-\delta_{n 1}\left[\left\{N_{2}, a_{1}^{+}\right\}, a_{m}^{-}\right]\right),
\end{aligned}
$$

where in turn

$$
\begin{aligned}
{\left[\left\{L_{12}, a_{1}^{-}\right\}, a_{m}^{-}\right]=} & 2 \delta_{m 2}\left\{L_{12}, M_{12}\right\}+\delta_{m 2}\left\{a_{1}^{-}, a_{1}^{+}\right\} \\
& -\delta_{m 1}\left\{a_{1}^{-}, a_{2}^{+}\right\}, \\
{\left[\left\{L_{12}, a_{2}^{-}\right\}, a_{m}^{-}\right]=} & -2 \delta_{m 1}\left\{L_{12}, M_{12}\right\}+\delta_{m 2}\left\{a_{2}^{-}, a_{1}^{+}\right\} \\
& -\delta_{m 1}\left\{a_{2}^{-}, a_{2}^{+}\right\}, \\
{\left[\left\{N_{21}, a_{1}^{+}\right\}, a_{m}^{-}\right]=} & 2 \delta_{m 1}\left\{N_{21}, N_{1}\right\}+2 \delta_{m 2}\left\{N_{21}, N_{12}\right\} \\
& -\delta_{m 2}\left\{a_{1}^{+}, a_{1}^{-}\right\}, \\
{\left[\left\{N_{12}, a_{2}^{+}\right\}, a_{m}^{-}\right]=} & 2 \delta_{m 1}\left\{N_{12}, N_{21}\right\}+2 \delta_{m 2}\left\{N_{12}, N_{2}\right\} \\
& -\delta_{m 1}\left\{a_{2}^{+}, a_{2}^{-}\right\}, \\
{\left[\left\{N_{1}, a_{2}^{+}\right\}, a_{m}^{-}\right]=} & 2 \delta_{m 1}\left\{N_{1}, N_{21}\right\}+2 \delta_{m 2}\left\{N_{1}, N_{2}\right\} \\
& -\delta_{m 1}\left\{a_{2}^{+}, a_{1}^{-}\right\}, \\
{\left[\left\{N_{2}, a_{1}^{+}\right\}, a_{m}^{-}\right]=} & 2 \delta_{m 1}\left\{N_{2}, N_{1}\right\}+2 \delta_{m 2}\left\{N_{2}, N_{12}\right\} \\
& -\delta_{m 2}\left\{a_{1}^{+}, a_{2}^{-}\right\} .
\end{aligned}
$$

Substituting these expressions into (G.4) and collecting similar terms, we find

$$
\begin{aligned}
& {\left[\left[a_{0}, a_{n}^{+}\right], a_{m}^{-}\right] } \\
&=-\frac{1}{2}\left\{\delta_{n 2} \delta_{m 1}\left(\left\{N_{21}, N_{1}\right\}-\left\{N_{1}, N_{21}\right\}\right)\right. \\
&+\delta_{n 2} \delta_{m 2}\left(\left\{L_{12}, M_{12}\right\}+\left\{N_{21}, N_{12}\right\}-\left\{N_{1}, N_{2}\right\}\right) \\
&+\delta_{n 1} \delta_{m 1}\left(\left\{L_{12}, M_{12}\right\}+\left\{N_{12}, N_{21}\right\}-\left\{N_{2}, N_{1}\right\}\right) \\
&\left.+\delta_{n 1} \delta_{m 2}\left(\left\{N_{12}, N_{2}\right\}-\left\{N_{2}, N_{12}\right\}\right)\right\}+ \\
&-\frac{1}{4}\left\{\delta_{n 2} \delta_{m 1}\left(-\left\{a_{1}^{-}, a_{2}^{+}\right\}+\left\{a_{2}^{+}, a_{1}^{-}\right\}\right)\right. \\
&+\delta_{n 2} \delta_{m 2}\left(\left\{a_{1}^{+}, a_{1}^{-}\right\}-\left\{a_{1}^{+}, a_{1}^{-}\right\}\right) \\
&+\delta_{n 1} \delta_{m 1}\left(\left\{a_{2}^{-}, a_{2}^{+}\right\}-\left\{a_{2}^{+}, a_{2}^{-}\right\}\right) \\
&\left.+\delta_{n 1} \delta_{m 2}\left(\left\{a_{2}^{-}, a_{1}^{+}\right\}+\left\{a_{1}^{+}, a_{2}^{-}\right\}\right)\right\} \\
&=-\frac{1}{2}(-4)\left(\delta_{n 2} \delta_{m 2}+\delta_{n 1} \delta_{m 1}\right) a_{0} \equiv 2 \delta_{n m} a_{0},
\end{aligned}
$$

which is the required result.

\section{Appendix H: Corrected Harish-Chandra's formula (68)}

Here, we give a derivation of the formula (68) from the paper [6] with a proper number coefficients of the matrices

$$
B_{r}=\sum_{\left(k_{1} \ldots k_{r}\right)} \beta_{k_{1}}^{2} \ldots \beta_{k_{1}}^{2},
$$

where the summation is to be taken over all possible values of $k_{1}, \ldots, k_{r}$ such that no two of $k^{\prime} \mathrm{s}$ are the same. In this Appendix we follow the notations accepted in [6].

The original formula (68) in [6] has the following form:

$\omega^{2}=(-1)^{\left[\frac{1}{2} s(s-1)\right]}\left[\frac{s+1}{2}\right]\left[\frac{s}{2}\right] \ldots\left[\frac{2}{2}\right]$ 


$$
\begin{aligned}
& \times\left\{B_{\left[\frac{1}{2}(s+1)\right]}-\left[\frac{s}{2}\right] B_{\left[\frac{1}{2}(s+3)\right]}+\frac{1}{2 !}\left[\frac{s}{2}\right]\right. \\
& \times\left[\frac{s-2}{2}\right] B_{\left[\frac{1}{2}(s+5)\right]}-\frac{1}{3 !}\left[\frac{s}{2}\right]\left[\frac{s-2}{2}\right]\left[\frac{s-4}{2}\right] \\
& \times B_{\left[\frac{1}{2}(s+7)\right]}+\cdots+(-1) \\
& \left.\left[\frac{1}{2} s\right]_{B_{s}}\right\} .
\end{aligned}
$$

Here, $s$ is space-time dimensions, $[n]$ denotes the integral part of $n$. This expression is derived from the following formula (Eq. (67) in [6]):

$$
\begin{aligned}
\omega^{2}= & (-1)\left[\frac{1}{2} s(s-1)\right]\left[\frac{s+1}{2}\right]\left[\frac{s}{2}\right] \ldots\left[\frac{2}{2}\right] \\
& \times P_{\left(k_{1} \ldots k_{r}\right)} \beta_{k_{s}}^{2} \beta_{k_{s-2}}^{2} \ldots\left(1-\beta_{k_{s-1}}^{2}\right)\left(1-\beta_{k_{s-3}}^{2}\right) \ldots,
\end{aligned}
$$

where $P_{\left(k_{1} \ldots k_{r}\right)}$ denotes a sum over all permutations of $(1,2, \ldots, s)$ and $k_{1}, \ldots, k_{s}$ are all different. In turn, (H.3) is a result of a product of two (non-normalized) matrices $\omega=\epsilon^{k_{1} \ldots k_{s}} \beta_{k_{1}} \ldots \beta_{k_{\mathrm{s}}}$. (H.3):

Let us remove the parentheses on the right-hand side of

$$
\begin{aligned}
& \omega^{2}=(-1)\left[\frac{1}{2} s(s-1)\right]\left[\frac{s+1}{2}\right]\left[\frac{s}{2}\right] \ldots\left[\frac{2}{2}\right] P_{\left(k_{1} \ldots k_{r}\right)} \\
& \times\left\{C_{0}^{[s / 2]} \beta_{k_{s}}^{2} \beta_{k_{s-2}}^{2} \ldots \beta_{k_{2}}^{2} 1_{k_{s-1}} 1_{k_{s-3}} \ldots 1_{k_{1}}\right. \\
& \text { - } C_{1}^{[s / 2]} \beta_{k_{s}}^{2} \beta_{k_{s-2}}^{2} \ldots \beta_{k_{2}}^{2} \beta_{k_{s-1}}^{2} 1_{k_{s-3}} 1_{k_{s-5}} \ldots 1_{k_{1}} \\
& +C_{2}^{[s / 2]} \beta_{k_{s}}^{2} \beta_{k_{s-2}}^{2} \ldots \beta_{k_{2}}^{2} \beta_{k_{s-1}}^{2} \beta_{k_{s-3}}^{2} 1_{k_{s-5}} 1_{k_{s-7}} \ldots 1_{k_{1}} \\
& \text { - } C_{3}^{[s / 2]} \beta_{k_{s}}^{2} \beta_{k_{s-2}}^{2} \ldots \beta_{k_{2}}^{2} \beta_{k_{s-1}}^{2} \beta_{k_{s-3}}^{2} \beta_{k_{s-5}}^{2} 1_{k_{s-7}} 1_{k_{s-9}} \ldots 1_{k_{1}} \\
& \left.\left.+\ldots+(-1)^{\left[\frac{1}{2} s\right.}\right]_{C_{[s / 2]}^{[s / 2]}} \beta_{k_{s}}^{2} \beta_{k_{s-2}}^{2} \ldots \beta_{k_{2}}^{2} \beta_{k_{s-1}}^{2} \beta_{k_{s-3}}^{2} \ldots \beta_{k_{1}}^{2}\right\} \text {. }
\end{aligned}
$$

Here, $C_{k}^{n}$ are binomial coefficients. Writing out their explicit form

$$
\begin{aligned}
& C_{0}^{[s / 2]}=1, C_{1}^{[s / 2]}=\frac{1}{1 !}\left[\frac{s}{2}\right], C_{2}^{[s / 2]}=\frac{1}{2 !}\left[\frac{s}{2}\right]\left[\frac{s-2}{2}\right], \\
& C_{3}^{[s / 2]}=\frac{1}{3 !}\left[\frac{s}{2}\right]\left[\frac{s-2}{2}\right]\left[\frac{s-4}{2}\right], \ldots,
\end{aligned}
$$

using the definition of matrices $B_{r}$, Eq. (H.1), and omitting a product of the unity matrices $1_{k_{s}}$, we reproduce the original formula of Harish-Chandra (H.2). However, indeed we have to take into account a possibility of rearrangement of the unity matrices $1_{k_{s-l}}, l=1,3,5, \ldots$. We explicitly set the markers $k_{s-l}$ for the unity matrices to emphasize the importance of accounting their rearrangements. This gives additional factors. So in the first term in braces in (H.4) the rearrangement of the unity matrices gives an additional factor $[s / 2]$ !, in the second term it gives the factor $[(s-2) / 2]$ ! etc. By this mean, as a result, instead of (H.2) now we have

$\omega^{2}=(-1)^{\left[\frac{1}{2} s(s-1)\right]}\left[\frac{s+1}{2}\right]\left[\frac{s}{2}\right] \ldots\left[\frac{2}{2}\right]$

$$
\begin{aligned}
& \times\left\{\left(\left[\frac{s}{2}\right] !\right) B_{\left[\frac{1}{2}(s+1)\right]}-\left[\frac{s}{2}\right]\left(\left[\frac{s-2}{2}\right] !\right) B_{\left[\frac{1}{2}(s+3)\right]}\right. \\
& +\frac{1}{2 !}\left[\frac{s}{2}\right]\left[\frac{s-2}{2}\right]\left(\left[\frac{s-4}{2}\right] !\right) B_{\left[\frac{1}{2}(s+5)\right]} \\
& -\frac{1}{3 !}\left[\frac{s}{2}\right]\left[\frac{s-2}{2}\right]\left[\frac{s-4}{2}\right]\left(\left[\frac{s-6}{2}\right] !\right) B_{\left[\frac{1}{2}(s+7)\right]}+\ldots \\
& \left.+(-1)\left[\frac{1}{2} s\right]_{B_{s}}\right\}
\end{aligned}
$$

or taking the common factorial $([s / 2]$ !) outside braces, we get

$$
\begin{aligned}
& \omega^{2}=(-1)\left[\frac{1}{2} s(s-1)\right]\left[\frac{s+1}{2}\right]\left[\frac{s}{2}\right] \ldots\left[\frac{2}{2}\right]\left(\left[\frac{s}{2}\right] !\right) \\
&\left\{B_{\left[\frac{1}{2}(s+1)\right]}-\frac{1}{1 !} B_{\left[\frac{1}{2}(s+3)\right]}+\frac{1}{2 !} B_{\left[\frac{1}{2}(s+5)\right]}\right. \\
& \quad-\frac{1}{3 !} B_{\left[\frac{1}{2}(s+7)\right]}+\ldots+(-1)\left[\frac{1}{2} s\right] \\
&\left.\quad \times\left(\left[\frac{s}{2}\right] !\right)^{-1} B_{s}\right\} .
\end{aligned}
$$

For the special case $s=4$ from this expression follows

$\omega^{2}=4\left\{2 B_{2}-2 B_{3}+B_{4}\right\}$,

while the original formula (H.2) gives us

$\omega^{2}=4\left\{B_{2}-2 B_{3}+B_{4}\right\}$.

As previously discussed, in Sect. 19, the formula (H.2) has never been used further in the text of the paper [6], except deriving the next formula (69) having the following form:

$$
\begin{aligned}
\omega^{3}= & (-1)\left[\frac{1}{2} s(s-1)\right] \\
& \times\left\{\left[\frac{s+1}{2}\right]\left[\frac{s}{2}\right]\left[\frac{s-1}{2}\right] \ldots\left[\frac{2}{2}\right]\right\}^{2} \omega .
\end{aligned}
$$

Let us show that this expression can not be reproduced by using the original formula (H.2), whereas the revised formula (H.5) do this.

We will need the following matrix relation [6]:

$\omega B=\left[\frac{s+1}{2}\right] \omega$.

We have used the special case of this formula in Sect. 19, Eq. (19.12). Besides, instead of the initial definition of matrices $B_{r}$, Eq. (H.1), we make use of the following representation:

$B_{r}=B(B-1) \ldots(B-r+1)$.

Let us multiply (H.8) by $\omega$ from the left. Taking into account (H.7), we find

$\omega B_{r}=\left[\frac{s+1}{2}\right]\left(\left[\frac{s+1}{2}\right]-1\right)$ 


$$
\times\left(\left[\frac{s+1}{2}\right]-2\right) \ldots\left(\left[\frac{s+1}{2}\right]-r+1\right) \omega .
$$

At a certain value $r \equiv r_{*}$ this expression for $r \geq r_{*}$ vanishes. This value equals

$r_{*}=\left[\frac{s+1}{2}\right]+1=\left[\frac{s+3}{2}\right]$

Further, we multiply the expression (H.2) by $\omega$. We see that all terms except the first one, by virtue of the condition (H.9) vanish and consequently we get

$$
\begin{aligned}
\omega^{3}= & (-1)\left[\frac{1}{2} s(s-1)\right] \\
& \times\left(\left[\frac{s+1}{2}\right]\left[\frac{s}{2}\right]\left[\frac{s-1}{2}\right] \ldots\left[\frac{2}{2}\right]\right) \omega B_{\left[\frac{1}{2}(s+1)\right]} \\
= & (-1)\left[\frac{1}{2} s(s-1)\right]\left(\left[\frac{s+1}{2}\right]\left[\frac{s}{2}\right]\left[\frac{s-1}{2}\right] \ldots\left[\frac{2}{2}\right]\right) \\
& \times\left(\left[\frac{s+1}{2}\right]\left[\frac{s-1}{2}\right]\left[\frac{s-3}{2}\right] \ldots\left[\frac{2}{2}\right]\right) \omega . \quad(\text { H.10 }
\end{aligned}
$$

Obviously, the formula (H.6) is not reproduced, because the factors $[s / 2],[(s-2) / 2],[(s-4) / 2], \ldots$ are lacking.

Now we multiply the revised formula (H.5) by the matrix $\omega$. Then instead of (H.10), we have

$$
\begin{aligned}
\omega^{3}= & (-1)\left[\frac{1}{2} s(s-1)\right] \\
& \times\left(\left[\frac{s+1}{2}\right]\left[\frac{s}{2}\right]\left[\frac{s-1}{2}\right] \ldots\left[\frac{2}{2}\right]\right)\left(\left[\frac{s}{2}\right] !\right) \\
& \times\left(\left[\frac{s+1}{2}\right]\left[\frac{s-1}{2}\right]\left[\frac{s-3}{2}\right] \ldots\left[\frac{2}{2}\right]\right) \omega .
\end{aligned}
$$

Here, in contrast to (H.10), an additional factor appears

$$
\left[\frac{s}{2}\right] !=\left[\frac{s}{2}\right]\left[\frac{s-2}{2}\right]\left[\frac{s-4}{2}\right] \ldots\left[\frac{2}{2}\right],
$$

which gives us the missing multiplies. Thereby the formula (H.5) reproduces (H.6).

\section{Appendix I: Proof of the relation $\left[a_{0}, \hat{\Lambda}\right]=0$}

In this Appendix we will give a proof of the second relation in (19.14). Let us present it as a sum of two terms

$\left[a_{0}, \hat{\Lambda}_{1}\right]+\left[a_{0}, \hat{\Lambda}_{2}\right]=0$

where $\hat{\Lambda}=\left\{a_{k}^{+}, a_{k}^{-}\right\}, k=1,2$. Substituting an explicit form of the operator $a_{0}$, Eq. (6.17), we present the first term in (I.1) in the following form:

$$
\begin{aligned}
- & \frac{1}{4}\left(\left[\left\{L_{12}, M_{12}\right\},\left\{a_{1}^{+}, a_{1}^{-}\right\}\right]+\left[\left\{N_{12}, N_{21}\right\},\left\{a_{1}^{+}, a_{1}^{-}\right\}\right]\right. \\
& \left.-\left[\left\{N_{1}, N_{2}\right\},\left\{a_{1}^{+}, a_{1}^{-}\right\}\right]\right) .
\end{aligned}
$$

Let us consider the first contribution in (I.2). Making use of the operator identity (19.17), we present it in the form:

$$
\begin{aligned}
{\left[\left\{L_{12}, M_{12}\right\},\left\{a_{1}^{+}, a_{1}^{-}\right\}\right]=} & \left\{L_{12},\left[M_{12},\left\{a_{1}^{+}, a_{1}^{-}\right\}\right]\right\} \\
& +\left\{M_{12},\left[L_{12},\left\{a_{1}^{+}, a_{1}^{-}\right\}\right]\right\},
\end{aligned}
$$

where in turn

$$
\begin{aligned}
{\left[M_{12},\left\{a_{1}^{+}, a_{1}^{-}\right\}\right] } & =-\left\{a_{1}^{+},\left[a_{1}^{-}, M_{12}\right]\right\}+\left\{a_{1}^{-},\left[M_{12}, a_{1}^{+}\right]\right\} \\
& =-\left\{a_{1}^{-}, a_{2}^{-}\right\}
\end{aligned}
$$

and

$$
\begin{aligned}
{\left[L_{12},\left\{a_{1}^{+}, a_{1}^{-}\right\}\right] } & =-\left\{a_{1}^{+},\left[a_{1}^{-}, L_{12}\right]\right\}+\left\{a_{1}^{-},\left[L_{12}, a_{1}^{+}\right]\right\} \\
& =-\left\{a_{1}^{+}, a_{2}^{+}\right\} .
\end{aligned}
$$

Here, we have used the commutation rules (6.15). As a result, the first contribution in (I.2) takes the form

$$
\begin{aligned}
& {\left[\left\{L_{12}, M_{12}\right\},\left\{a_{1}^{+}, a_{1}^{-}\right\}\right]} \\
& \quad=-\left\{L_{12},\left\{a_{1}^{-}, a_{2}^{-}\right\}\right\}-\left\{M_{12},\left\{a_{1}^{+}, a_{2}^{+}\right\}\right\} .
\end{aligned}
$$

For the second contribution in (I.2) a similar reasoning results in

$$
\begin{aligned}
& {\left[\left\{N_{12}, N_{21}\right\},\left\{a_{1}^{+}, a_{1}^{-}\right\}\right]} \\
& \quad=\left\{N_{12},\left\{a_{1}^{-}, a_{2}^{-}\right\}\right\}-\left\{N_{21},\left\{a_{1}^{+}, a_{2}^{+}\right\}\right\},
\end{aligned}
$$

and the third contribution in (I.2) vanishes.

Further, we transform the right-hand side of (I.3). For the first term on the right-hand side we make use of the operator identity (19.16)

$$
\begin{aligned}
\left\{L_{12},\left\{a_{1}^{-}, a_{2}^{-}\right\}\right\} & =\left[a_{1}^{-},\left[a_{2}^{-}, L_{12}\right]\right]+\left\{a_{2}^{-},\left\{L_{12}, a_{1}^{-}\right\}\right\} \\
& =2 N_{1}+\left\{a_{2}^{-},\left\{L_{12}, a_{1}^{-}\right\}\right\} .
\end{aligned}
$$

Here, we have employed again the commutation rules (6.15). For the second term in (I.3) we have similarly

$$
\left\{M_{12},\left\{a_{1}^{-}, a_{2}^{-}\right\}\right\}=-2 N_{1}+\left\{a_{2}^{+},\left\{M_{12}, a_{1}^{+}\right\}\right\},
$$

and then (I.3) goes into

$$
\begin{aligned}
{\left[\left\{L_{12}, M_{12}\right\},\left\{a_{1}^{+}, a_{1}^{-}\right\}\right]=} & -\left\{a_{2}^{-},\left\{L_{12}, a_{1}^{-}\right\}\right\} \\
& -\left\{a_{2}^{+},\left\{M_{12}, a_{1}^{+}\right\}\right\} .
\end{aligned}
$$

We make another transformation of the right-hand side of the last expression with the aim that instead of the generators $L_{12}$ and $M_{12}$ the second pair of the generators $N_{12}$ and $N_{21}$ has appeared. By using the identity (19.17) for the internal anticommutator in the first term in (I.5) and in the definition of the generator $L_{12}$, we obtain

$$
\begin{aligned}
\left\{L_{12}, a_{1}^{-}\right\} & =\frac{1}{2}\left\{\left[a_{1}^{+}, a_{2}^{+}\right], a_{1}^{-}\right\} \\
& =\frac{1}{2}\left(\left\{a_{1}^{+},\left[a_{2}^{+}, a_{1}^{-}\right]\right\}-\left[a_{2}^{+},\left\{a_{1}^{-}, a_{1}^{+}\right\}\right]\right) \\
& =\left\{a_{1}^{+}, N_{21}\right\}-\frac{1}{2}\left[a_{2}^{+},\left\{a_{1}^{-}, a_{1}^{+}\right\}\right] .
\end{aligned}
$$


A similar transformation for the internal anticommutator in the second term in (I.5) gives

$\left\{M_{12}, a_{1}^{+}\right\}=-\left\{a_{1}^{-}, N_{12}\right\}-\frac{1}{2}\left[a_{2}^{-},\left\{a_{1}^{+}, a_{1}^{-}\right\}\right]$,

and the right-hand side of (I.5) becomes

$$
\begin{aligned}
- & \left\{a_{2}^{-},\left\{a_{1}^{+}, N_{21}\right\}\right\}+\left\{a_{2}^{+},\left\{a_{1}^{-}, N_{12}\right\}\right\} \\
& +\frac{1}{2}\left(\left\{a_{2}^{-},\left[a_{2}^{+},\left\{a_{1}^{-}, a_{1}^{+}\right\}\right]\right\}-\left\{a_{2}^{+},\left[a_{2}^{-},\left\{a_{1}^{+}, a_{1}^{-}\right\}\right]\right\}\right) .
\end{aligned}
$$

The expression in parentheses here can be written in a more compact form:

$\left[\left\{a_{2}^{+}, a_{2}^{-}\right\},\left\{a_{1}^{+}, a_{1}^{-}\right\}\right] \equiv\left[\hat{\Lambda}_{2}, \hat{\Lambda}_{1}\right]$

and then the first contribution in (I.2) takes its final form

$$
\begin{aligned}
{\left[\left\{L_{12}, M_{12}\right\},\left\{a_{1}^{+}, a_{1}^{-}\right\}\right] } & =-\left\{a_{2}^{-},\left\{a_{1}^{+}, N_{21}\right\}\right\} \\
& +\left\{a_{2}^{+},\left\{a_{1}^{-}, N_{12}\right\}\right\}+\frac{1}{2}\left[\hat{\Lambda}_{2}, \hat{\Lambda}_{1}\right] .
\end{aligned}
$$

It is sufficient to transform the right-hand side of the second contribution (I.4) to the form similar to (I.5). Then instead of (I.4) we have

$$
\begin{aligned}
{\left[\left\{N_{12}, N_{21}\right\},\left\{a_{1}^{+}, a_{1}^{-}\right\}\right]=} & \left\{a_{2}^{+},\left\{N_{12}, a_{1}^{-}\right\}\right\} \\
& -\left\{a_{2}^{-},\left\{N_{21}, a_{1}^{+}\right\}\right\} .
\end{aligned}
$$

We should note that here the signs on the right-hand side coincide with the signs of the first two terms in (I.6) contrary to the expectations. Summing (I.6) and (I.7), we get

$$
\begin{aligned}
{\left[a_{0}, \hat{\Lambda}_{1}\right]=} & \frac{1}{2}\left(\left\{a_{2}^{-},\left\{a_{1}^{+}, N_{21}\right\}\right\}-\left\{a_{2}^{+},\left\{a_{1}^{-}, N_{12}\right\}\right\}\right) \\
& +\frac{1}{8}\left[\hat{\Lambda}_{1}, \hat{\Lambda}_{2}\right] .
\end{aligned}
$$

As we see, this expression does not vanish. The second term on the left-hand side of (I.1) can be obtained from the previous one by a simple replacement of indices $1 \rightleftarrows 2$ (the operator $a_{0}$ is invariant with respect to such a replacement, and $N_{12} \rightleftarrows N_{21}$ ):

$$
\begin{aligned}
{\left[a_{0}, \hat{\Lambda}_{2}\right]=} & \frac{1}{2}\left(\left\{a_{1}^{-},\left\{a_{2}^{+}, N_{12}\right\}\right\}-\left\{a_{1}^{+},\left\{a_{2}^{-}, N_{21}\right\}\right\}\right) \\
& -\frac{1}{8}\left[\hat{\Lambda}_{1}, \hat{\Lambda}_{2}\right] .
\end{aligned}
$$

We sum the last two expressions and, finally, obtain

$$
\begin{aligned}
& {\left[a_{0}, \hat{\Lambda}_{1}+\hat{\Lambda}_{2}\right]} \\
& =\frac{1}{2}\left(\left\{a_{2}^{-},\left\{a_{1}^{+}, N_{21}\right\}\right\}-\left\{a_{1}^{+},\left\{a_{2}^{-}, N_{21}\right\}\right\}\right) \\
& \quad+\frac{1}{2}\left(\left\{a_{1}^{-},\left\{a_{2}^{+}, N_{12}\right\}\right\}-\left\{a_{2}^{+},\left\{a_{1}^{-}, N_{12}\right\}\right\}\right) \\
& =\frac{1}{2}\left(\left[N_{21},\left[a_{1}^{+}, a_{2}^{-}\right]\right]+\left[N_{12},\left[a_{2}^{+}, a_{1}^{-}\right]\right]\right)
\end{aligned}
$$

$$
\equiv\left[N_{21}, N_{12}\right]+\left[N_{12}, N_{21}\right]=0 .
$$

By this mean, in contrast to (19.23), the relation (I.1) is fulfilled only for a sum of two terms $\hat{\Lambda}_{1}$ and $\hat{\Lambda}_{2}$ and therefore the equalities (19.24) are not the case.

\section{References}

1. R.J. Duffin, On the characteristic matrices of covariant systems Phys. Rev. 54, 1114 (1938)

2. N. Kemmer, The particle aspect of meson theory. Proc. R. Soc. A 173, 91-116 (1939)

3. G. Petiau, Contribution à l'étude des équations d'ondes corpusculaires, Ph.D. Thesis, University of Paris (1936)

4. G. Petiau, Acad. R. de Belg. A. Sci. Mem. Collect. XVI, 118 (1936)

5. N. Kemmer, The algebra of meson matrices. Math. Proc. Camb. Philos. Soc. 39, 189-196 (1943)

6. Harish-Chandra, On relativistic wave equations. Phys. Rev. 71, 793-805 (1947)

7. I. Fujiwara, On the Duffin-Kemmer algebra. Prog. Theor. Phys. 10, 589-616 (1953)

8. Z. Tokuoka, H. Tanaka, On the equivalence of the particle formalism and the wave formalism of meson. Prog. Theor. Phys. 8, 599-614 (1952)

9. Z. Tokuoka, Generating relations of the sub-algebras in the DuffinKemmer algebra. Nucl. Phys. 78, 681-693 (1966)

10. N.A. Chernikov, The Fock representation of the Duffin-Kemmer algebra. Acta Phys. Polon. 21, 51-60 (1962)

11. E. Fischbach, M.M. Nieto, C.K. Scott, Duffin-Kemmer-Petiau subalgebras: representations and applications. J. Math. Phys. 14, 1760-1774 (1973)

12. E. Fischbach, J.D. Louck, M.M. Nieto, C.K. Scott, The Lie algebra $s o(N)$ and the Duffin-Kemmer-Petiau ring. J. Math. Phys. 15, 60 64 (1974)

13. A.T. Filippov, A.P. Isaev, A.B. Kurdikov, Paragrassmann differential calculus. Theor. Math. Phys. 94, 150-165 (1993)

14. A.P. Isaev, Paragrassmann integral, discrete systems and quantum groups. Int. J. Mod. Phys. A 12, 201-206 (1997)

15. H. Umezawa, Quantum Field Theory (North-Holland Publishing Company, Amsterdam, 1956)

16. S. Okubo, Supergeneralization of Duffin-Kemmer-Petiau algebra and Lie superalgebra $\operatorname{osp}(N, M)$. J. Math. Phys. 42, 4554-4562 (2001)

17. D.V. Volkov, On the quantization of half-integer spin fields. Sov. Phys. JETP 9, 1107-1111 (1959)

18. C. Ryan, E.C.G. Sudarshan, Representations of parafermi rings. Nucl. Phys. 47, 207-211 (1963)

19. N.N. Bogolyubov, A.A. Logunov, A.I. Oksac, I.T. Todorov, General Principles of Quantum Field Theory (Kluwer Academic Publishers, Dordrecht, 1989)

20. YuA Markov, M.A. Markova, D.M. Gitman, Unitary quantization and para-Fermi statistics of order 2. J. Exp. Theor. Phys. 127, 398$421(2018)$

21. S. Kamefuchi, Y. Takahashi, A generalization of field quantization and statistics. Nucl. Phys. 36, 177-206 (1962)

22. B. Geyer, On the generalization of canonical commutation relations with respect to the orthogonal group in even dimensions. Nucl. Phys. B 8, 326-332 (1968)

23. E. Schrödinger, Pentads, tetrads, and triads of meson-matrices. Proc. R. Irish Acad. A 48, 135-146 (1943)

24. YuA Markov, M.A. Markova, A.I. Bondarenko, Third order wave equation in Duffin-Kemmer-Petiau theory: massive case. Phys. Rev. D 92, 105017 (2015) 
25. M. Omote, S. Kamefuchi, Para-Grassmann algebras and paraFermi systems. Lett. Nuovo Cimento 24, 345-350 (1979)

26. Y. Ohnuki, S. Kamefuchi, Para-Grassmann algebras with applications to para-Fermi systems. J. Math. Phys. 21, 609-616 (1980)

27. A.J. Kálnay, A note on Grassmann algebras. Rep. Math. Phys. 9, 9-13 (1976)

28. F. Bayen, M. Flato, C. Fronsdal, A. Lichnerowicz, D. Sternheimer, Deformation theory and quantization. II. Physical applications. Ann. Phys. 110, 111-151 (1978)

29. I.V. Tyutin, General form of the $*$-commutator on the Grassmann algebra. Theor. Math. Phys. 128, 1271-1292 (2001)

30. A.V. Smilga, Quasiciassical expansion for $\operatorname{Tr}\left\{(-1)^{F} \mathrm{e}^{-\beta H}\right\}$. Commun. Math. Phys. 230, 245-269 (2002)

31. A.C. Hirshfeld, P. Henselder, Deformation quantization for systems with fermions. Ann. Phys. 302, 59-77 (2002)

32. M. Daoud, Covariance of the Grassmann star product. Rep. Math. Phys. 52, 281-294 (2003)

33. A.K. Kwasniewski, J. Math. Phys. 26, 2234-2238 (1985)

34. L. Baulieu, E.G. Floratos, Phys. Lett. B 258, 171-178 (1991)

35. N. Fleury, M. Rausch de Traubenberg, J. Math. Phys. 33, 33563366 (1992)

36. M. Rausch de Traubenberg, Adv. Appl. Clifford Algebras 4, 131144 (1994)

37. A.T. Filippov, A.P. Isaev, A.B. Kurdikov, Mod. Phys. Lett. A 7, 2129-2141 (1992)

38. A.T. Filippov, A.P. Isaev, A.B. Kurdikov, Int. J. Mod. Phys. A 8, 4973-5003 (1993)

39. A.T. Filippov, A.P. Isaev, A.B. Kurdikov, Theor. Math. Phys. 94, 150-165 (1993)

40. A.T. Filippov, A.P. Isaev, A.B. Kurdikov, in Problems in Modern Theoretical Physics. Dubna 96-212, p. 83 (1996) (in Russian)

41. YuA Markov, M.A. Markova, A.I. Bondarenko, Fourth-order wave equation in Bhabha-Madhavarao spin-3/2 theory. Int. J. Mod. Phys. A 32, 1750144 (2017)

42. M. Omote, Y. Ohnuki, S. Kamefuchi, Fermi-Bose similarity. Prog. Theor. Phys. 56, 1948-1964 (1976)

43. A.J. Bracken, H.S. Green, Identities for para-Fermi statistics of given order. Nuovo Cimento A 9, 349-365 (1972)

44. M.D. Gould, J. Paldus, Para-Fermi algebras and the many-electron correlation problem. Phys. Rev. A 34, 804-814 (1986)

45. H. Fukutome, The group theoretical structure of fermion manybody systems arising from the canonical anticommutation relation. I. Prog. Theor. Phys. 65, 809-827 (1981)

46. H.S. Green, A generalized method of field quantization. Phys. Rev. 90, 270-273 (1953)
47. Harish-Chandra, The correspondence between the particle and the wave aspects of the meson and the photon. Proc. R. Soc. A 186, 502-525 (1946)

48. Harish-Chandra, On the algebra of the meson matrices. Proc. Camb. Philos. Soc. 43, 414-421 (1947)

49. N.V. Borisov, P.P. Kulish, Path integral in superspace for a relativistic spinor particle in an external gauge field. Theor. Math. Phys. 51, 535-540 (1982)

50. Ju.L. Daleckii, M.G. Krein, Stability of Solutions of Differential Equations in Banach Space (American Mathematical Society, Providence, 1974)

51. Y. Ohnuki, S. Kamefuchi, Some general properties of para-Fermi field theory. Phys. Rev. 170, 1279-1293 (1968)

52. Y. Ohnuki, S. Kamefuchi, Wavefunctions of identical particles. Ann. Phys. (N.Y.) 51, 337-358 (1969)

53. Y. Ohnuki, S. Kamefuchi, The statistical quantum number and gauge groups in parafermi field theory. Ann. Phys. (N.Y.) 78, 64-89 (1973)

54. Y. Ohnuki, S. Kamefuchi, Parafermi field theory and elementary particles. Prog. Theor. Phys. 52, 1369-1375 (1974)

55. M. Hama, M. Sawamura, H. Suzuki, Pair mode in two-level parafermion systems. Europhys. Lett. 21(4), 383-387 (1993)

56. B.S. Madhava Rao, Commutation rules for matrices related to particles of higher spins. Proc. Indian Acad. Sci. A 15, 139-147 (1942)

57. B.S. Madhava Rao, V.R. Thiruvenkatachar, K. Venkatachaliengar, Algebra related to elementary particles of spin 3/2. Proc. R. Soc. A 187, 385-397 (1946)

58. R.A. Krajcik, M.M. Nieto, Bhabha first-order wave equations: I. $C, P$, and $T$. Phys. Rev. D 10, 4049-4063 (1974)

59. F.A. Dilkes, D.G.C. McKeon, C. Schubert, A new approach to axial vector model calculations II. JHEP 03, 022 (1999)

60. E.D. D'Hoker, D.G. Gagné, Worldline path integrals for fermions with scalar, pseudoscalar and vector couplings. Nucl. Phys. B 467, 272-296 (1996)

61. A.P. Prudnikov, YuA Brychkov, O.I. Marichev, Integrals and Series: Elementary Functions, vol. 1 (Gordon and Breach, Amsterdam, 1986)

62. F. Gliozzi, J. Scherk, D. Olive, Supersymmetry, supergravity theories and the dual spinor model. Nucl. Phys. B 122, 253-290 (1977)

63. Yu.A. Markov, M.A. Markova, Star product for para-Grassmann algebra of order two (in preparation)

64. S. Jing, C.A. Nelson, Eigenstates of paraparticle creation operators. J. Phys. A Math. Gen. 32, 401-409 (1999) 